\title{
Physical and chemical differentiation of the luminous star-forming region $\mathrm{W}_{49 A^{\star}}$
}

\section{Results from the JCMT Spectral Legacy Survey}

\author{
Z. Nagy ${ }^{1,2,3,4}$, F. F. S. van der Tak ${ }^{3,2}$, G. A. Fuller ${ }^{5}$, and R. Plume ${ }^{6}$ \\ 1 I. Physikalisches Institut, Universität zu Köln, Zülpicher Str. 77, 50937 Köln, Germany \\ e-mail: zsofia.nagy.astro@gmail.com \\ 2 Kapteyn Astronomical Institute, University of Groningen, PO Box 800, 9700 AV Groningen, The Netherlands \\ 3 SRON Netherlands Institute for Space Research, PO Box 800, 9700 AV Groningen, The Netherlands \\ ${ }_{4}$ Department of Physics and Astronomy, University of Toledo, 2801 West Bancroft Street, Toledo, OH 43606, USA \\ 5 Jodrell Bank Centre for Astrophysics, School of Physics and Astronomy, University of Manchester, Manchester, M13 9PL, UK \\ ${ }^{6}$ Department of Physics and Astronomy, University of Calgary, Calgary, T2N 1N4, AB, Canada
}

Received 17 September 2014 / Accepted 10 February 2015

\section{ABSTRACT}

\begin{abstract}
Context. The massive and luminous star-forming region W49A is a well-known Galactic candidate to probe the physical conditions and chemistry similar to those expected in external starburst galaxies.

Aims. We aim to probe the physical and chemical structure of W49A on a spatial scale of $\sim 0.8 \mathrm{pc}$ based on the JCMT Spectral Legacy Survey, which covers the frequency range between 330 and $373 \mathrm{GHz}$.

Methods. The wide $2 \times 2$ arcmin field and the high spectral resolution of the HARP instrument on JCMT provides information on the spatial structure and kinematics of the cloud traced by the observed molecular lines. For species where multiple transitions are available, we estimate excitation temperatures and column densities using a population diagram method that takes beam dilution and optical depth corrections into account.

Results. We detected 255 transitions corresponding to 63 species in the 330-373 GHz range at the center position of W49A. Excitation conditions can be probed for 14 molecules, including the complex organic molecules $\mathrm{CH}_{3} \mathrm{CCH}, \mathrm{CH}_{3} \mathrm{CN}$, and $\mathrm{CH}_{3} \mathrm{OH}$. The chemical composition suggests the importance of shock, photon-dominated region (PDR), and hot core chemistry. Many molecular lines show a significant spatial extent across the maps including $\mathrm{CO}$ and its isotopologues, high density tracers (e.g., $\mathrm{HCN}, \mathrm{HNC} \mathrm{CS}, \mathrm{HCO}^{+}$), and tracers of UV irradiation (e.g., $\mathrm{CN}$ and $\mathrm{C}_{2} \mathrm{H}$ ). The spatially extended species reveal a complex velocity-structure of W49A with possible infall and outflow motions. Large variations are seen between the subregions with mostly blue-shifted emission toward the eastern tail, mostly red-shifted emission toward the northern clump, and emission peaking around the expected source velocity toward the southwest clump.

Conclusions. A comparison of column density ratios of characteristic species observed toward W49A to Galactic PDRs suggests that while the chemistry toward the W49A center is driven by a combination of UV irradiation and shocks, UV irradiation dominates for the northern clump, eastern tail, and southwest clump regions. A comparison to a starburst galaxy and an active galactic nucleus suggests similar $\mathrm{C}_{2} \mathrm{H}, \mathrm{CN}$, and $\mathrm{H}_{2} \mathrm{CO}$ abundances (with respect to the dense gas tracer ${ }^{34} \mathrm{CS}$ ) between the $\sim 0.8$ pc scale probed for W49A and the $>1 \mathrm{kpc}$ regions in external galaxies with global star formation.
\end{abstract}

Key words. stars: formation - ISM: molecules

\section{Introduction}

Star formation in galaxies occurs on different scales, from isolated cores/globules with sizes of $<1 \mathrm{pc}$ through giant molecular clouds of a few $10 \mathrm{pc}$ up to the scales of starburst galaxies representing star formation on scales of a few $100 \mathrm{pc}$, and with luminosities of $L_{\mathrm{IR}}>10^{11} L_{\odot}$ for luminous infrared galaxies (LIRGs) and $L_{\mathrm{IR}}>10^{12} L_{\odot}$ for ultraluminous infrared galaxies (ULIRGs). These regions also represent a large spread in star formation rates with the $3 M_{\odot} / \mathrm{yr}$ in the Milky Way to $\sim 10^{3}$ $M_{\odot} / \mathrm{yr}$ in starburst galaxies (e.g., Solomon \& Vanden Bout 2005). The different types of star-forming regions in galaxies may trace different physical processes that control star formation in these different environments.

\footnotetext{
* Appendices are available in electronic form at http://www. aanda.org
}

The Galactic starburst analogue W49A, with its more than $\sim 100$ pc size (Simon et al. 2001), represents an intermediate case between the star formation seen in Galactic star-forming regions and in starburst galaxies. With its intense star formation compared to other Galactic sources, studying its physical and chemical properties can give insights into the mechanism behind the starburst phenomenon. In our previous work based on part of the data presented in this paper, we focused on the physical properties of W49A (Nagy et al. 2012). In this work we focus more on the chemistry of this Galactic starburst analogue. W49A is one of the most massive $\left(M \sim 10^{6} M_{\odot}\right.$, Sievers et al. 1991) and luminous $\left(>10^{7} L_{\odot}\right.$, Ward-Thompson \& Robson 1990) star-forming regions in the Galaxy. Even though its luminosity is lower than that of starburst galaxies, W49A is more comparable to ULIRGs in terms of its luminosity per unit mass of gas. In ULIRGs values of $\sim 100 L_{\odot} / M_{\odot}$ are measured 
(e.g., Solomon et al. 1997), while the value for W49A is $>10 L_{\odot} / M_{\odot}$. The luminosity of W49A is a result of an embedded stellar cluster containing the equivalent of about $100 \mathrm{O} 7$ stars (Conti \& Blum 2002), corresponding to four stellar clusters in a $5^{\prime} \times 5^{\prime}(16 \times 16 \mathrm{pc})$ region around the center based on $J, H$, and $K_{\mathrm{S}}$ images (Alves \& Homeier 2003). Wu et al. (2014) discovered a very massive star corresponding to the central cluster of $\mathrm{W} 49 \mathrm{~A}$, and estimated its mass to be in the range between 90 and $250 M_{\odot}$. Apart from the stellar population observed at near-infrared wavelengths, the ongoing star formation, which appears to be forming a comparable mass of stars, can be studied in the radio continuum (e.g., De Pree et al. 1997, 2004, 2005) and at mm- and sub-mm (e.g., Wilner et al. 2001; Galvan-Madrid et al. 2013) wavelengths as hot cores and ultracompact (UC) HII regions.

The distance of W49A was recently estimated to be $11.11_{-0.69}^{+0.79} \mathrm{kpc}$ (Zhang et al. 2013), which is consistent with the $11.4 \mathrm{kpc}$ value derived by Gwinn et al. (1992). Because of the large distance of W49A, its small-scale structure, including hot cores, outflows, and UC HII regions, require interferometric observations to be fully resolved (e.g., Wilner et al. 2001; De Pree et al. 2005; Galvan-Madrid et al. 2013). However, previous studies using single-dish telescopes have already provided information on the chemical complexity of $\mathrm{W} 49 \mathrm{~A}$, and the physical and chemical properties of the region.

$\mathrm{HCO}^{+}$1-0 lines with red-shifted absorption and blue-shifted emission were observed by Welch et al. (1987) and interpreted as evidence of global collapse toward the central $\sim 2 \mathrm{pc}$ region of W49A. An alternative explanation for the large number of O-stars contributing to the luminosity of W49A was proposed by Serabyn et al. (1993), who suggested a cloud-cloud collision based on multiple transitions of CS and $C^{34} S$ (from $J=3-2$ to $J=10-9$ ). Based on more recent observations, however, this explanation is less probable than the global cloud collapse scenario. Large-scale maps of W49A (e.g., Galvan-Madrid et al. 2013) show a hierarchical network of filaments, converging from larger scales to the center of W49A (W49N).

Signatures of the high star formation activity were discovered using Spitzer mid-IR images and position-velocity diagrams based on ${ }^{13} \mathrm{CO} J=2-1$ and $\mathrm{C}^{18} \mathrm{O} J=2-1$ maps by Peng et al. (2010), who have identified two expanding shells toward W49A.

Vastel et al. (2001) studied the physical conditions in the photon-dominated region (PDR) component of W49A using the FIR lines of [OI] and [CII] observed with the Long Wavelength Spectrometer of the Infrared Space Observatory. They also studied the rotational lines of $\mathrm{CO}\left(\mathrm{CO} 1-0, \mathrm{C}^{18} \mathrm{O} 2-1\right)$ observed with the $15-\mathrm{m}$ SEST telescope, and derived a radiation field of $G_{0}=3 \times 10^{5}$ (in units of $1.3 \times 10^{-4} \mathrm{erg} \mathrm{s}^{-1} \mathrm{~cm}^{-2} \mathrm{sr}^{-1}$ ) and an average gas density of $10^{4} \mathrm{~cm}^{-3}$.

A recent study based on data from the JCMT Spectral Legacy Survey (SLS, Plume et al. 2007) focused on an extended warm and dense gas component toward W49A seen in $\mathrm{H}_{2} \mathrm{CO}$ (Nagy et al. 2012), and characterized the physical properties and excitation of the region using $\mathrm{CH}_{3} \mathrm{OH}, \mathrm{SO}_{2}, \mathrm{H}_{2} \mathrm{CO}$, and $\mathrm{HCN}$ transitions. Another study used the SLS line survey data to focus on dense gas tracers (Roberts et al. 2011), such as HCN, HNC, $\mathrm{DCN}, \mathrm{HCO}^{+}$, and their isotopologues, and compared the line ratios of these gas tracers to line ratios measured toward starburst galaxies and active galactic nuclei (AGN), as well as to chemical models, and found that W49A is a template for starburst galaxies rather than for AGNs.

In this paper, we present results on the chemical inventory of W49A based on the SLS, carried out with the James
Clerk Maxwell Telescope (JCMT) at a resolution of $\sim 15^{\prime \prime}$. This line survey provides the largest frequency coverage data toward W49A to date, and as such, it provides a useful starting point for future higher resolution studies with instruments such as ALMA.

\section{Observations and data reduction}

We carried out the SLS observations using the 16-receptor (spatial pixel) Heterodyne Array Receiver Programme B (HARP$\mathrm{B}, 325-375 \mathrm{GHz}$ ) and the Auto-Correlation Spectral Imaging System (ACSIS) correlator (Buckle et al. 2009) at the James Clerk Maxwell Telescope ${ }^{1}$ (JCMT) on Mauna Kea, Hawaii. We carried out the observations in jiggle position switch mode, sampled every $7.5^{\prime \prime}$ for a $2 \times 2$ arcmin field centered on RA(J2000) $=$ $19^{\mathrm{h}} 10^{\mathrm{m}} 13^{\mathrm{s}} .4 ; \operatorname{Dec}(\mathrm{J} 2000)=09^{\circ} 06^{\prime} 14^{\prime \prime}$. We calibrated the spectra using an off-position corresponding to an offset $\left(+840^{\prime \prime},+840^{\prime \prime}\right)$ compared to the central position. We carried out the observations toward the reference position to confirm that the off position is reliable and is without emission at the observed frequencies. We checked the pointing every hour and estimated that it is accurate to $1.5^{\prime \prime}$. The angular resolution of the JCMT is $\sim 15^{\prime \prime}$ at $345 \mathrm{GHz}$, equivalent to $\sim 0.8 \mathrm{pc}$ at the distance of $\mathrm{W} 49$. The spectral resolution at the observed frequencies is $\sim 0.8 \mathrm{~km} \mathrm{~s}^{-1}$ and the beam efficiency is 0.63 (Buckle et al. 2009). The original line survey was carried out in the $330-360 \mathrm{GHz}$ frequency range and was later extended to $373 \mathrm{GHz}$. This paper summarizes the results from the whole $330-373 \mathrm{GHz}$ line survey.

We reduced the data using a combination of tasks from the Starlink package and the ORAC Data Reduction pipeline (ORAC-DR). We reduced the observed time-series cubes using the ORAC-DR pipeline, which creates three-dimensional cubes after checking for consistency between the calculated $T_{\text {sys }}$ and observed rms noise in the data; checking for variations in the rms noise measured by each receptor across the map, removes baselines from every spectrum and coadds spectra that corresponds to the same position and frequency. The results of the pipeline were checked and were corrected for remaining bad data, such as spectra with high rms noise level, bad baselines, and spectral ranges affected by spikes, using a combination of Starlink tasks.

Figure 1 shows the typical rms noise levels as a function of frequency in the $330-373 \mathrm{GHz}$ frequency range measured at every $2 \mathrm{GHz}$. Typical noise levels in $T_{\mathrm{A}}^{*}$ units are in the range between 0.02 and $0.12 \mathrm{~K}$. The highest noise level measured at $\sim 368 \mathrm{GHz}$ is due to the poor atmospheric transmission as shown in the top panel of Fig. 1. Atmospheric transmissions are shown for typical precipitable water vapor (PWV) levels of $1 \mathrm{~mm}$ and $3.75 \mathrm{~mm}$ on Mauna Kea.

\section{Results}

\subsection{Line identification}

We have identified the lines detected toward the center of W49A $\left(\operatorname{RA}(J 2000)=19^{\mathrm{h}} 10^{\mathrm{m}} 13.4 ; \operatorname{Dec}(\mathrm{J} 2000)=09^{\circ} 06^{\prime} 14^{\prime \prime}\right)$ in the frequency range between 330 and $373 \mathrm{GHz}$. Analyzed positions other then the center include the eastern tail $\left(\operatorname{RA}(J 2000)=19^{\mathrm{h}} 10^{\mathrm{m}} 16^{\mathrm{s}} \cdot 6 ; \operatorname{Dec}(\mathrm{J} 2000)=09^{\circ} 05^{\prime} 48^{\prime \prime}\right)$, northern clump $\left(\mathrm{RA}(\mathrm{J} 2000)=19^{\mathrm{h}} 10^{\mathrm{m}} 13.6 ; \operatorname{Dec}(\mathrm{J} 2000)=09^{\circ} 06^{\prime} 48^{\prime \prime}\right)$,

1 The James Clerk Maxwell Telescope is operated by the Joint Astronomy Centre on behalf of the Science and Technology Facilities Council of the United Kingdom, the Netherlands Organisation for Scientific Research, and the National Research Council of Canada. 


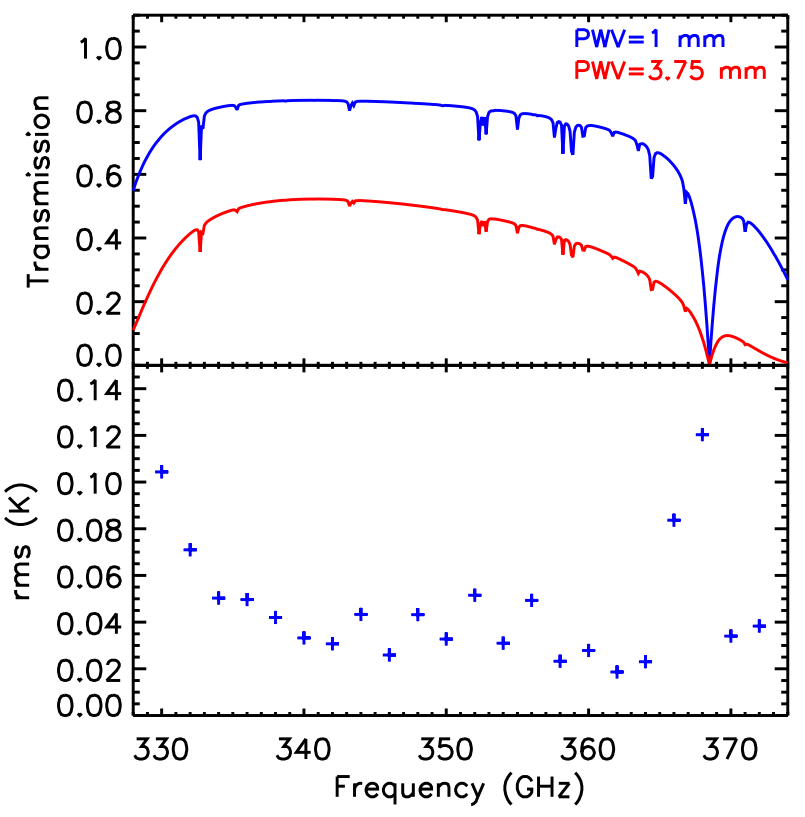

Fig. 1. Typical rms noise levels in $T_{\mathrm{A}}^{*}$ units measured in the SLS survey as a function of the frequency (bottom panel) and the corresponding atmospheric transmission for typical levels of water vapor at Mauna Kea (top panel).

and southwest clump $\left(\mathrm{RA}(\mathrm{J} 2000)=19^{\mathrm{h}} 10^{\mathrm{m}} 10 \mathrm{~s} \cdot 6 ; \operatorname{Dec}(\mathrm{J} 2000)=\right.$ $\left.09^{\circ} 05^{\prime} 18^{\prime \prime}\right)$. We selected these positions based on different kinematical signatures traced by dense gas tracers such as $\mathrm{HCO}^{+}$, HCN, and HNC (Roberts et al. 2011), and are shown for example in Fig. 3. Appendix A shows details on the detected species. We have added a comment when the lines were detected in the high-mass protostar AFGL 2591, another source in the JCMT SLS, based on Van der Wiel (2011). The spectroscopic data are based on the Cologne Database for Molecular Spectroscopy (CDMS, Müller et al. 2005) ${ }^{2}$ and the Jet Propulsion Laboratory (JPL, Pickett et al. 1998) ${ }^{3}$ molecular databases. The identification is based on an initial search range of transitions with an upper level energy of $400 \mathrm{~K}$, and was extended in particular cases such as vibrationally excited lines of HCN and HNC. In the case of weak ( $\$ 3 \sigma$ detections) lines, an additional check of the spatial distribution and a cross-check of the detection in data corresponding to different rest frequencies was applied to avoid possible artifacts, e.g., spurs or baseline errors. For the identification, we considered velocities in a range between $\pm 5 \mathrm{~km} \mathrm{~s}^{-1}$ around the expected source velocity of $10 \mathrm{~km} \mathrm{~s}^{-1}$ and extended it for the most asymmetric lines.

Toward the center position we detected about 255 lines in the $330-373 \mathrm{GHz}$ frequency range, which belong to 63 molecular species summarized in Table 1 . Most of the detected lines are related to sulphur-bearing species, such as $\mathrm{SO}_{2}$ and its isotopologues. Various species are detected that indicate the possible importance of shock and hot core chemistry and UV irradiation (see Sect. 4). The detected transitions cover a large energy range up to $\sim 1067 \mathrm{~K}$, corresponding to vibrationally excited HCN (Fuller et al., in prep.). However, most transitions have upper level energies below $400 \mathrm{~K}$ (Fig. 2). Excitation conditions can be probed for 16 species (Table 2) based on multiple detected transitions with a sufficient energy range. Among the $\sim 255$ detected lines, there are three unidentified lines

\footnotetext{
2 http://www.astro.uni-koeln.de/cdms/catalog

3 http://spec.jpl.nasa.gov
}

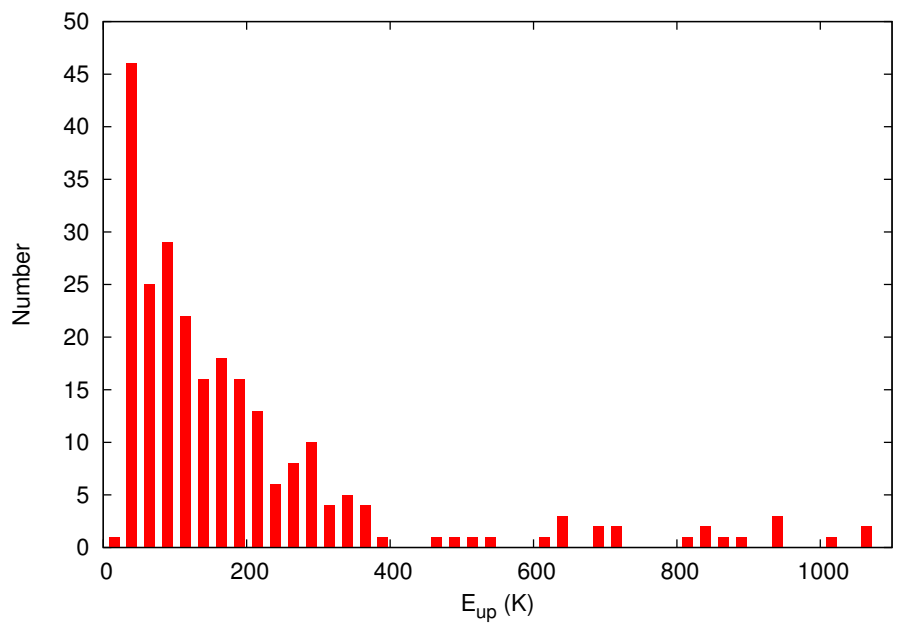

Fig. 2. Number of transitions detected versus the upper state energy of the transition in $25 \mathrm{~K}$ wide bins.

(U-lines in Appendix A.1). With the large number of spectral features, mis-assignment to species is possible. Also some of the line profiles are non-Gaussian, and the full width at half maximum (FWFM) widths of the lines vary over a relatively large range ( $\sim 7-19 \mathrm{~km} \mathrm{~s}^{-1}$ toward the central position). This may suggest that some of the identified lines are unresolved blends and contribute to the line emission from other molecules or transitions. However the excitation diagram analysis provides a crosscheck on the line identifications (and indeed highlighted some initial misassignments), and we believe that misindentified lines are not a signifiant issue.

To measure the spatial extent of the detected species, we apply a two-dimensional Gaussian fit to the integrated line intensities, using the BEAMFIT task in Starlink. The values shown in Table 1 correspond to the FWHM values of the fit. For molecules with multiple detected transitions, we include their largest spatial extent. For molecules with measured source sizes of $\sim 23 \times 23^{\prime \prime}$, the lines were only detected toward a few positions around the source center. In the following sections we quote sizes of $\sim 17 \times 17^{\prime \prime}$ for these species, to correct for the $\sim 15^{\prime \prime}$ beam size of JCMT around the observed frequencies. The center positions of the Gaussian fits, corresponding to the different species, are within a few arcseconds distance around the W49A center. In addition to $\mathrm{CO}$ and its isotopologues, high density tracers (e.g., $\mathrm{HCN}, \mathrm{HNC}, \mathrm{CS}, \mathrm{HCO}^{+}$) and tracers of UV-irradiation (e.g., $\mathrm{CN}$ and $\mathrm{C}_{2} \mathrm{H}$ ) show the largest spatial extent (Table 1). The measured position angles cover a large range indicating a complex source structure. Section 3.2 includes a summary of the detected molecules. We discuss the excitation conditions and column densities of the species with multiple detected transitions in Sect. 3.4.

\subsection{The detected species}

The detected species summarized in Table 1 can be related to various processes including shocks, UV irradiation by the embedded OB stars, and hot core chemistry, which includes complex organic molecules that are released from the grains into the gas-phase at temperatures of $100-300 \mathrm{~K}$ and are shielded from the dissociative UV radiation.

\subsubsection{Shock tracers}

A large number of the detected molecules can be related to shock chemistry (Fig. 3). All of these molecules are observed toward 
Table 1. The measured spatial extent of the species detected toward the center of W49A.

\begin{tabular}{|c|c|c|c|c|c|c|c|c|c|c|c|}
\hline \multirow[t]{2}{*}{ Species } & \multirow{2}{*}{$\begin{array}{l}\text { Number of } \\
\text { transitions }\end{array}$} & \multirow{2}{*}{$\begin{array}{l}E_{\text {up }} \\
(\mathrm{K})\end{array}$} & \multicolumn{3}{|c|}{ Spatial extent ${ }^{a}$} & \multirow[t]{2}{*}{ Species } & \multirow{2}{*}{$\begin{array}{l}\text { Number of } \\
\text { transitions }\end{array}$} & \multirow{2}{*}{$\begin{array}{l}E_{\text {up }} \\
(\mathrm{K})\end{array}$} & \multicolumn{3}{|c|}{ Spatial extent ${ }^{a}$} \\
\hline & & & $\begin{array}{l}\text { Major } \\
\text { axis (") }\end{array}$ & $\begin{array}{l}\text { Minor } \\
\text { axis (") }\end{array}$ & $\begin{array}{r}\text { Pos. angle } \\
\text { (deg) }\end{array}$ & & & & $\begin{array}{l}\text { Major } \\
\text { axis (") }\end{array}$ & $\begin{array}{l}\text { Minor } \\
\text { axis (") }\end{array}$ & $\begin{array}{r}\text { Pos. angle } \\
\text { (deg) }\end{array}$ \\
\hline $\mathrm{CO}$ & 1 & 33 & 98 & 60 & 90 & ${ }^{13} \mathrm{CO}$ & 1 & 32 & 72 & 54 & 87 \\
\hline $\mathrm{C}^{17} \mathrm{O}$ & 1 & 32 & 39 & 28 & 69 & $\mathrm{C}_{2} \mathrm{H}$ & 3 & 42 & 61 & 46 & 79 \\
\hline $\mathrm{CN}$ & 3 & 33 & 54 & 39 & 77 & $\mathrm{HCN}$ & 1 & 43 & 37 & 27 & 76 \\
\hline CS & 1 & 66 & 39 & 24 & 70 & $\mathrm{DCN}$ & 1 & 52 & 36 & 23 & 146 \\
\hline $\mathrm{CH}_{3} \mathrm{OH}$ & 26 & $17-372$ & 30 & 23 & 64 & $\mathrm{H}_{2} \mathrm{CO}$ & 7 & $52-241$ & 29 & 23 & 64 \\
\hline $\mathrm{C}^{34} \mathrm{~S}$ & 1 & 65 & 28 & 23 & 69 & $\mathrm{H}_{2} \mathrm{CS}$ & 6 & 91-209 & 30 & 23 & 142 \\
\hline NS & 1 & 70 & 29 & 23 & 9 & NO & 3 & $36-209$ & 29 & 23 & 62 \\
\hline${ }^{33} \mathrm{SO}$ & 3 & $78-87$ & 33 & 23 & 112 & $\mathrm{HNC}$ & 1 & 43 & 31 & 23 & 67 \\
\hline $\mathrm{HCO}^{+}$ & 1 & 43 & 32 & 26 & 179 & $\mathrm{H}$ & 2 & & 35 & 23 & 77 \\
\hline $\mathrm{SO}_{2}$ & 41 & $31-612$ & 23 & 23 & & $\mathrm{~N}_{2} \mathrm{H}^{+}$ & 1 & 45 & 23 & 23 & \\
\hline${ }^{33} \mathrm{SO}_{2}$ & 18 & $35-338$ & 23 & 23 & & ${ }^{13} \mathrm{CS}$ & 1 & 80 & 23 & 23 & \\
\hline${ }^{34} \mathrm{SO}_{2}$ & 33 & $35-547$ & 23 & 23 & & $\mathrm{H}_{3} \mathrm{O}^{+}$ & 1 & 140 & 23 & 23 & \\
\hline $\mathrm{SO}^{18} \mathrm{O}$ & 4 & $117-326$ & 23 & 23 & & $\mathrm{SiO}$ & 1 & 75 & 23 & 23 & \\
\hline $\mathrm{SO}^{17} \mathrm{O}$ & 1 & $58-180$ & 23 & 23 & & $\mathrm{SO}_{2}, v_{2}=1$ & 9 & $805-998$ & 23 & 23 & \\
\hline${ }^{13} \mathrm{CH}_{3} \mathrm{OH}$ & 1 & 45 & 23 & 23 & & SO & 6 & $26-143$ & 23 & 23 & \\
\hline $\mathrm{HCS}^{+}$ & 1 & 74 & 23 & 23 & & ${ }^{34} \mathrm{SO}$ & 5 & $25-85$ & 23 & 23 & \\
\hline $\mathrm{H}^{13} \mathrm{CN}$ & 1 & 41 & 23 & 23 & & OCS & 3 & $237-271$ & 25 & 23 & \\
\hline $\mathrm{SO}^{+}$ & 1 & 70 & 23 & 23 & & $\mathrm{HNCO}$ & 7 & $127-204$ & 23 & 23 & \\
\hline $\mathrm{O}^{13} \mathrm{C}^{34} \mathrm{~S}$ & 1 & 247 & 23 & 23 & & $\mathrm{CH}_{3} \mathrm{CN}$ & 5 & $151-215$ & 23 & 23 & \\
\hline $\mathrm{HC}^{15} \mathrm{~N}$ & 1 & 41 & 23 & 23 & & $\mathrm{H}^{13} \mathrm{CO}^{+}$ & 1 & 42 & 23 & 23 & \\
\hline $\mathrm{HN}^{13} \mathrm{C}$ & 1 & 42 & 23 & 23 & & $\mathrm{CH}_{3} \mathrm{CCH}$ & 4 & $172-254$ & 23 & 23 & \\
\hline $\mathrm{HCN}, v_{2}=1$ & 1 & 1067 & 23 & 23 & & $\mathrm{HC}_{3} \mathrm{~N}$ & 3 & $307-376$ & 23 & 23 & \\
\hline $\mathrm{HC}_{3} \mathrm{~N}, v_{7}=1$ & 4 & $629-663$ & 23 & 23 & & $\mathrm{HCO}$ & 1 & 74 & 23 & 23 & \\
\hline $\mathrm{CH}_{3} \mathrm{OCH}_{3}$ & 2 & $48-49$ & 23 & 23 & & $\mathrm{~S}^{18} \mathrm{O}$ & 3 & 91-99 & 23 & 23 & \\
\hline $\mathrm{CO}^{+}$ & 1 & 34 & 23 & 23 & & $\mathrm{H}_{2} \mathrm{~S}$ & 2 & $135-263$ & 23 & 23 & \\
\hline $\mathrm{S}^{17} \mathrm{O}$ & 2 & $76-103$ & 23 & 23 & & $\mathrm{H}_{2}^{13} \mathrm{CO}$ & 2 & $61-65$ & 23 & 23 & \\
\hline $\mathrm{CH}_{3} \mathrm{CHO}$ & 1 & 155 & 23 & 23 & & $\mathrm{HNC}, v_{2}=1$ & 2 & $709-710$ & 23 & 23 & \\
\hline $\mathrm{H}^{15} \mathrm{NC}$ & 1 & 43 & 23 & 23 & & $\mathrm{HC}^{17} \mathrm{O}^{+}$ & 1 & 42 & 23 & 23 & \\
\hline $\mathrm{H}_{2} \mathrm{CN}$ & 3 & $53-100$ & 23 & 23 & & $\mathrm{HO}^{13} \mathrm{C}^{+}$ & 1 & 41 & 23 & 23 & \\
\hline $\mathrm{HC}^{18} \mathrm{O}^{+}$ & 1 & 41 & 23 & 23 & & $\mathrm{H}_{2} \mathrm{C}^{34} \mathrm{~S}$ & 1 & 118 & 23 & 23 & \\
\hline $\mathrm{CH}_{3} \mathrm{COCH}_{3}$ & 1 & 282 & 23 & 23 & & $\mathrm{H}_{2}^{33} \mathrm{~S}$ & 1 & 154 & 23 & 23 & \\
\hline $\mathrm{H}_{2}^{34} \mathrm{~S}$ & 1 & 154 & 23 & 23 & & & & & & & \\
\hline
\end{tabular}

Notes. The position angle is measured from north through east. ${ }^{(a)}$ The error corresponding to the fitted major and minor axis values is a few arcseconds. ${ }^{(b)}$ Position angles are only derived for species with significant spatial extent and elongation to minimize the uncertainty.

$\mathrm{a} \lesssim 20^{\prime \prime} \times 20^{\prime \prime}$ field around the center, but not detected toward the other subregions analyzed in this paper. The molecules related to shock chemistry include:

- SiO was detected in hot and shocked regions, such as molecular outflows (e.g., Nisini et al. 2007) and supernova remnants (e.g., Ziurys et al. 1989). The observations suggest that silicon is released from grain mantles in these regions. A process that can release silicon into the gas phase is the sputtering of (charged) grains by heavy neutral particles in C-shocks (Schilke et al. 1997; Gusdorf et al. 2008). SiO is detected toward W49A with a spatial extent of $\sim 17^{\prime \prime} \times 17^{\prime \prime}$ (corrected for the $15^{\prime \prime}$ beam size of JCMT), covering the positions corresponding to the central stellar cluster in its $J=8-7$ transition. The $\mathrm{SiO} J=2-1$ transition has previously been detected toward W49A by Lucas \& Liszt (2000).

- Most of the detected species are sulphur-bearing molecules, such as $\mathrm{SO}$ and $\mathrm{SO}_{2}$ and their isotopologues ${ }^{34} \mathrm{SO}_{2},{ }^{33} \mathrm{SO}_{2}$, $\mathrm{SO}^{17} \mathrm{O}, \mathrm{SO}^{18} \mathrm{O},{ }^{34} \mathrm{SO},{ }^{33} \mathrm{SO}, \mathrm{S}^{17} \mathrm{O}$, and $\mathrm{S}^{18} \mathrm{O}$. Sulphur is frozen onto grain mantles and can be sputtered in shocks, leading to the formation of the species mentioned above (e.g., Wakelam et al. 2004). We also detected nine transitions of vibrationally excited $\mathrm{SO}_{2}$. These molecules are only detected toward the center of W49A and spatially confined to a region of $\sim 17^{\prime \prime} \times 17^{\prime \prime}$, similar to $\mathrm{SiO}$.

- Other detected sulphur-bearing species include $\mathrm{H}_{2} \mathrm{~S}$, OCS (and its isotopologue $\mathrm{O}^{13} \mathrm{C}^{34} \mathrm{~S}$ ). $\mathrm{H}_{2} \mathrm{~S}$ and OCS show spatial extents similar to that of $\mathrm{SO}_{2}, \mathrm{SO}$, and their isotopologues; and to $\mathrm{SiO}$.

- We also detected $\mathrm{SO}^{+}$, which has been reported to be a diagnostic of dissociative shock chemistry (Turner 1992), but has also been detected toward various PDRs (see Sect. 3.2.2).

- We detected six transitions of HNCO, which has also been reported to trace shocks toward the Galactic center and in molecular outflows, such as L1157 (Rodríguez-Fernández et al. 2010).

\subsubsection{Tracers of UV irradiation}

The embedded clusters of $\mathrm{O}$ and B stars in the W49 region (e.g., Alves \& Homeier 2003) are expected to create PDRs. Several 
Z. Nagy et al.: Physical and chemical differentiation of W49A

Table 2. Results of the rotation and population diagram analysis for molecules with multiple transitions.

\begin{tabular}{lrlllll}
\hline \hline Species & $\begin{array}{r}T_{\text {rot }} \\
(\mathrm{K})\end{array}$ & $\begin{array}{l}N_{\text {tot }} \\
\left(\mathrm{cm}^{-2}\right)\end{array}$ & $\begin{array}{l}T_{\text {ex }} \\
(\mathrm{K})\end{array}$ & $\begin{array}{l}N_{\text {tot, }} \\
\left(\mathrm{cm}^{-2}\right)\end{array}$ & $\begin{array}{l}\text { Source size } \\
\left({ }^{\prime \prime}\right)\end{array}$ & Optical depth $^{c}$ \\
\hline $\mathrm{SO}_{2}{ }^{b}$ & $170 \pm 23$ & $(3.1 \pm 0.02) \times 10^{16}$ & $118 \pm 7$ & $2.1_{-0.0}^{+0.3} \times 10^{18}$ & $2.9_{-0.0}^{+0.0}$ & $0.1-9.3$ \\
${ }^{33} \mathrm{SO}_{2}$ & $105 \pm 18$ & $(1.3 \pm 0.01) \times 10^{15}$ & $95_{-8}^{+11}$ & $7.6_{-2.0}^{+1.1} \times 10^{17}$ & $1.0 \pm 0.0$ & $0.8-5.3$ \\
${ }^{34} \mathrm{SO}_{2}$ & $140 \pm 9$ & $(4.0 \pm 0.02) \times 10^{15}$ & $133 \pm 11$ & $4.8_{-4.3}^{+11.8} \times 10^{16}$ & $4.3_{-1.9}^{+9.3}$ & $0.01-0.2$ \\
$\mathrm{SO}_{2}, v_{2}=1$ & $146 \pm 26$ & $(1.9 \pm 0.06) \times 10^{17}$ & $143_{-31}^{+64}$ & $2.0_{-1.5}^{+500} \times 10^{17}$ & $14.5_{-13.5}^{+0.5}$ & $0.001-0.004$ \\
$\mathrm{CH}_{3} \mathrm{OH}^{b}$ & $75 \pm 5$ & $(7.7 \pm 0.04) \times 10^{15}$ & $65_{-2}^{+7}$ & $5.0_{-0.6}^{+0.0} \times 10^{17}$ & $2.4_{-0}^{+0}$ & $0.14-2.69$ \\
$\mathrm{H}_{2} \mathrm{CO}^{b}$ & $213 \pm 96$ & $(8.6 \pm 0.09) \times 10^{14}$ & $159_{-51}^{+90}$ & $1.4_{-1.2}^{+2.0} \times 10^{16}$ & $3.3_{-0.7}^{+4.9}$ & $0.10-1.50$ \\
$\mathrm{HNCO}$ & $61 \pm 20$ & $(4.9 \pm 0.2) \times 10^{14}$ & $54_{-34}^{+43}$ & $7.6_{-3.8}^{+6599} \times 10^{14}$ & $12.7_{-9.8}^{+2.3}$ & $0.01-0.05$ \\
$\mathrm{H}_{2} \mathrm{CS}$ & $196 \pm 85$ & $(1.6 \pm 0.02) \times 10^{14}$ & $195_{-97}^{+104}$ & $1.7_{-0.5}^{+723} \times 10^{14}$ & $14.5_{-13.5}^{+0.7}$ & $0.001-0.005$ \\
$\mathrm{CH}_{3} \mathrm{CN}$ & $70 \pm 30$ & $(1.1 \pm 0.1) \times 10^{13}$ & $69_{-27}^{+94}$ & $3.1_{-1.1}^{+16.9} \times 10^{14}$ & $3.0_{-1.6}^{+0.0}$ & $0.16-0.27$ \\
${ }^{34} \mathrm{SO}^{13}$ & $31 \pm 3$ & $(5.9 \pm 0.04) \times 10^{14}$ & $33_{-6}^{+8}$ & $6.2_{-1.2}^{+16.7} \times 10^{14}$ & $15.0_{-7.5}^{+0.0}$ & $0.01-0.22$ \\
$\mathrm{SO}^{18} \mathrm{O}$ & $196 \pm 26$ & $(8.4 \pm 0.05) \times 10^{14}$ & $173_{-105}^{+126}$ & $1.91_{-1.89}^{+7.4} \times 10^{17}$ & $1.0_{-0.0}^{+14.0}$ & $0.20-0.70$ \\
$\mathrm{CH}_{3} \mathrm{CCH}$ & $31 \pm 7$ & $(2.8 \pm 0.1) \times 10^{16}$ & $32 \pm 7$ & $2.6_{-1.4}^{+91} \times 10^{16}$ & $15.0_{-12.1}^{+0.0}$ & $0.01-0.03$ \\
$\mathrm{HC}_{3} \mathrm{~N}$ & $101 \pm 39$ & $(4.7 \pm 0.2) \times 10^{13}$ & $101^{a}$ & $4.8_{-0.6}^{+377} \times 10^{13}$ & $15.0_{-13.5}^{+0.0}$ & $0.004-0.006$ \\
$\mathrm{OCS}$ & $92 \pm 46$ & $(1.6 \pm 0.08) \times 10^{15}$ & $92^{a}$ & $1.7_{-0.3}^{+953} \times 10^{15}$ & $14.4_{-13.2}^{+0.6}$ & $0.008-0.010$ \\
\hline
\end{tabular}

Notes. ${ }^{(a)}$ Fixed parameter in the fit; ${ }^{(b)}$ Transitions in the frequency range between 360 and $373 \mathrm{GHz}$ were included in Nagy et al. (2012). ${ }^{(c)}$ The optical depth is calculated for each transition. Here we show the highest and lowest value for each molecule.

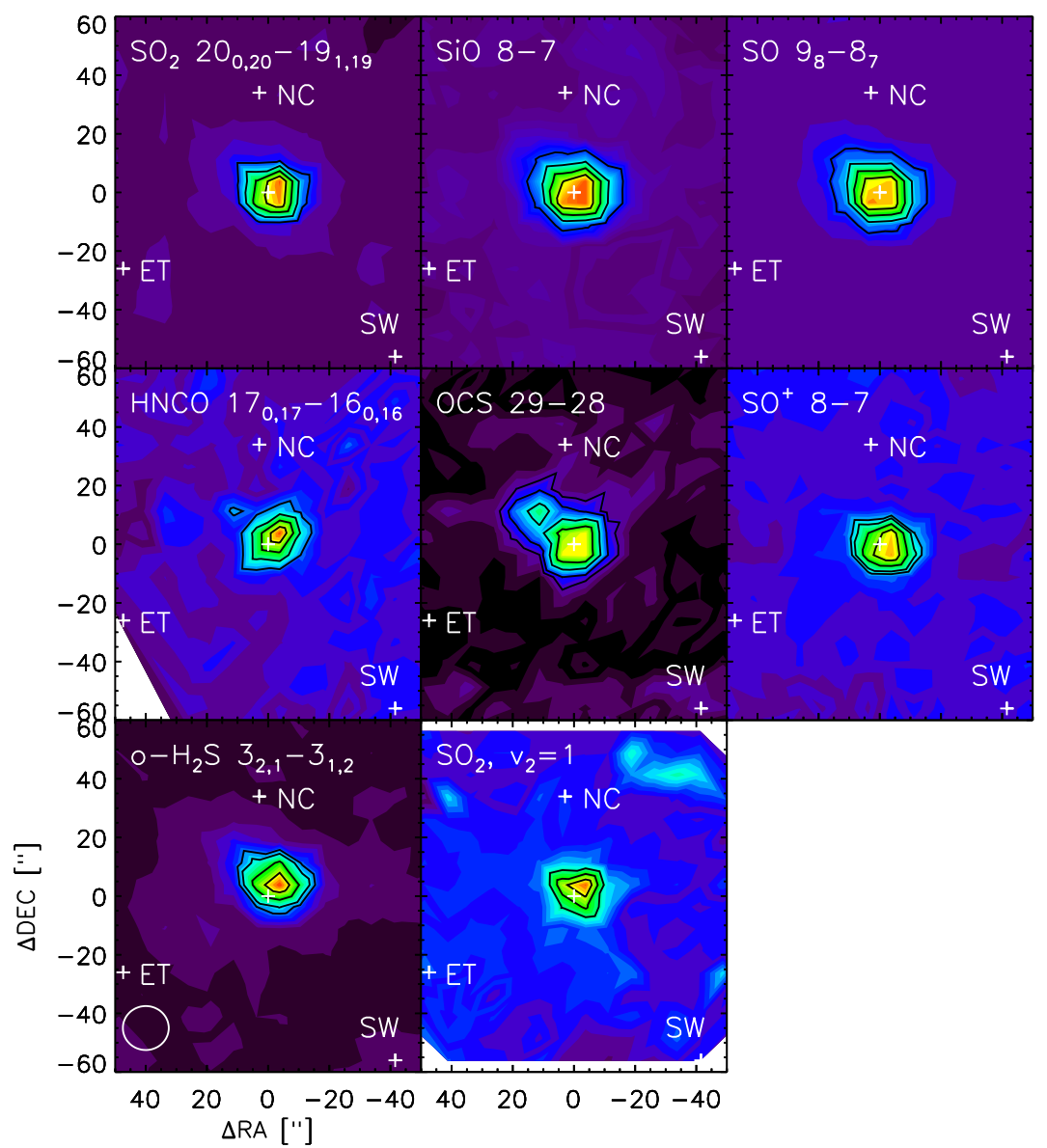

Fig. 3. Spatial distribution of species that can be related to shock chemistry. The contours correspond to the $20 \%, 40 \%, 60 \%$, and $80 \%$ of the maximum line intensity. The $\mathrm{SO}_{2}, v_{2}=1$ integrated intensity was calculated as the total integrated intensity of the three blended $\mathrm{SO}_{2}, v_{2}=1$ lines around $366.1 \mathrm{GHz}$. 


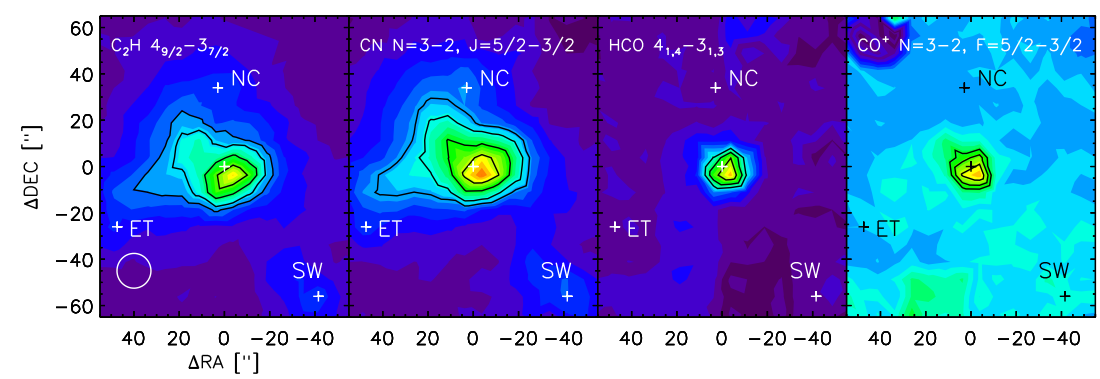

Fig. 4. Spatial distribution of species that can be related to PDR chemistry. For $\mathrm{C}_{2} \mathrm{H}$ and $\mathrm{CN}$, the contours correspond to the $30 \%$, $40 \%$, $60 \%$, and $80 \%$ of the maximum line intensity. For $\mathrm{CO}^{+}$and $\mathrm{HCO}$ the contours correspond to the $45 \%, 60 \%$, and $80 \%$ of the maximum line intensity. The different percentages used for the contour levels for the $\mathrm{CO}^{+}$and $\mathrm{HCO}$ integrated intensities is due to the lower signal-to-noise of these species compared to $\mathrm{C}_{2} \mathrm{H}$ and $\mathrm{CN}$.

species that can be related to PDR chemistry are detected in the SLS frequency range (Fig. 4), some of them with a significant spatial extent.

- We detected CN with a large extent $\left(\sim 54^{\prime \prime} \times 39^{\prime \prime}\right)$, including all subregions. This suggests the possible importance of PDR chemistry in W49A and may be tested using the ratio of $\mathrm{CN} / \mathrm{HCN}$, which is a well-known tracer of PDRs (e.g., Fuente et al. 1996). The spatial distribution of HCN and its isotopomer HNC based on SLS data were analyzed in Roberts et al. (2011).

- $\mathrm{C}_{2} \mathrm{H}$ (ethynyl) is a commonly observed molecule in the interstellar medium, including in PDRs. In star-forming regions $\mathrm{C}_{2} \mathrm{H}$ was proposed to be related to the earliest stages of massive star formation (Beuther et al. 2008). We detected three transitions toward W49A, with a very large spatial extent $\left(\sim 61^{\prime \prime} \times 46^{\prime \prime}\right)$.

- HCO (formyl radical) is also detected in a $\sim 17^{\prime \prime} \times 17^{\prime \prime}$ region around the center. $\mathrm{HCO}$ was found to be a tracer of illuminated cloud interfaces as it was detected in several PDRs, including the Orion Bar, NGC 2023, NGC 7023, and S140 (Schilke et al. 2001), and the Horsehead PDR (Gerin et al. 2009).

- The $\mathrm{SO}^{+}$and $\mathrm{CO}^{+}$reactive ions have been detected in various PDRs, such as the Orion Bar (Fuente et al. 2003), and in other regions of high FUV and X-ray irradiation. We detected both $\mathrm{CO}^{+}$and $\mathrm{SO}^{+}$toward the center of W49A with a spatial extent of $\sim 17^{\prime \prime} \times 17^{\prime \prime}$.

\subsubsection{Complex organic molecules}

We have detected a number of complex organic molecules (e.g., Herbst \& Van Dishoeck 2009), which are most likely related to hot cores.

- $\mathrm{CH}_{3} \mathrm{OH}$ is detected over a $30^{\prime \prime} \times 20^{\prime \prime}$ region around the center. Its spatial distribution is shown in Nagy et al. (2012). Its ${ }^{13} \mathrm{CH}_{3} \mathrm{OH}$ isotopologue is also detected.

- We detected five transitions of $\mathrm{CH}_{3} \mathrm{CN}$ (methyl cyanide, Fig. 5), which has been detected in hot cores, including Orion KL. The $J=12-11$ transition of $\mathrm{CH}_{3} \mathrm{CN}$ was previously detected toward W49 using the BIMA array and is likely connected to hot cores and also follows the compact dust concentrations (Wilner et al. 2001).

- We detected four transitions of $\mathrm{HC}_{3} \mathrm{~N}$ (cyanoacetylene, Fig. 5), which has been detected toward various Galactic star-forming regions as well as in a number of dust-rich galaxies (Lindberg et al. 2011). As it is destroyed by UV radiation and in reactions with the $\mathrm{C}^{+}$ion, $\mathrm{HC}_{3} \mathrm{~N}$ is expected

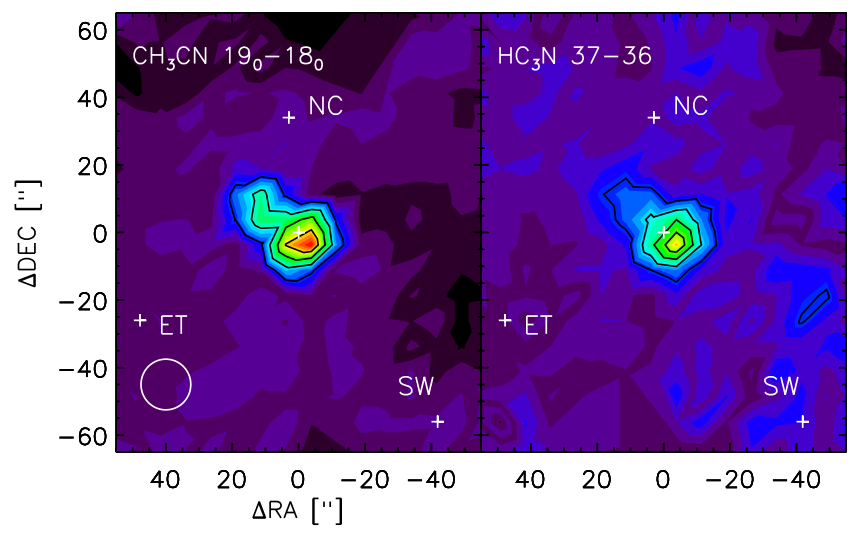

Fig. 5. Spatial distribution of the detected most spatially extended complex organic molecules. The crosses correspond to subregions: source center, eastern tail, northern clump, and southwest clump. The contours correspond to the $20 \%, 40 \%, 60 \%$, and $80 \%$ of the maximum line intensity.

to trace warm, dense, and shielded regions. This may explain the fact that $\mathrm{HC}_{3} \mathrm{~N}$ is seen to be confined toward the central $\sim 17^{\prime \prime} \times 17^{\prime \prime}$ region. Some of the vibrational transitions of $\mathrm{HC}_{3} \mathrm{~N}\left(v_{7}=1\right)$ have also been detected toward the W49A center. Vibrationally excited $\mathrm{HC}_{3} \mathrm{~N}$ has also been detected toward several hot cores (Wyrowski et al. 1999), as well as the galaxy NGC 4418 (Costagliola \& Aalto 2010).

- One transition of $\mathrm{CH}_{3} \mathrm{CHO}$ (acetaldehyde) is detected toward the W49A center. A previous survey by Ikeda et al. (2001) detected this molecule toward various hot molecular clouds.

- Four transitions of $\mathrm{CH}_{3} \mathrm{CCH}$ (propyne) are detected in our line survey at a few central pixels with a spatial extent of $\sim 17^{\prime \prime} \times 17^{\prime \prime}$. This molecule has been detected in a number of starburst galaxies, such as M82 (Aladro et al. 2011a).

\subsubsection{Other detected species}

- $\mathrm{CO},{ }^{13} \mathrm{CO}$, and $\mathrm{C}^{17} \mathrm{O}$ are covered by the SLS survey, and are detected at each position of the SLS field (Fig. 7), allowing us to probe the gas kinematics and column density.

- The only deuterated species we detected toward W49A is DCN (Roberts et al. 2011). An upper limit for the covered but not detected, $\mathrm{DCO}^{+} J=5-4$ transition was also reported by Roberts et al. (2011). A low abundance of deuterated ions is expected as deuterated molecular ions are destroyed rapidly at gas temperatures above $30 \mathrm{~K}$ (e.g., Roberts \& Millar 2007). 
Z. Nagy et al.: Physical and chemical differentiation of W49A

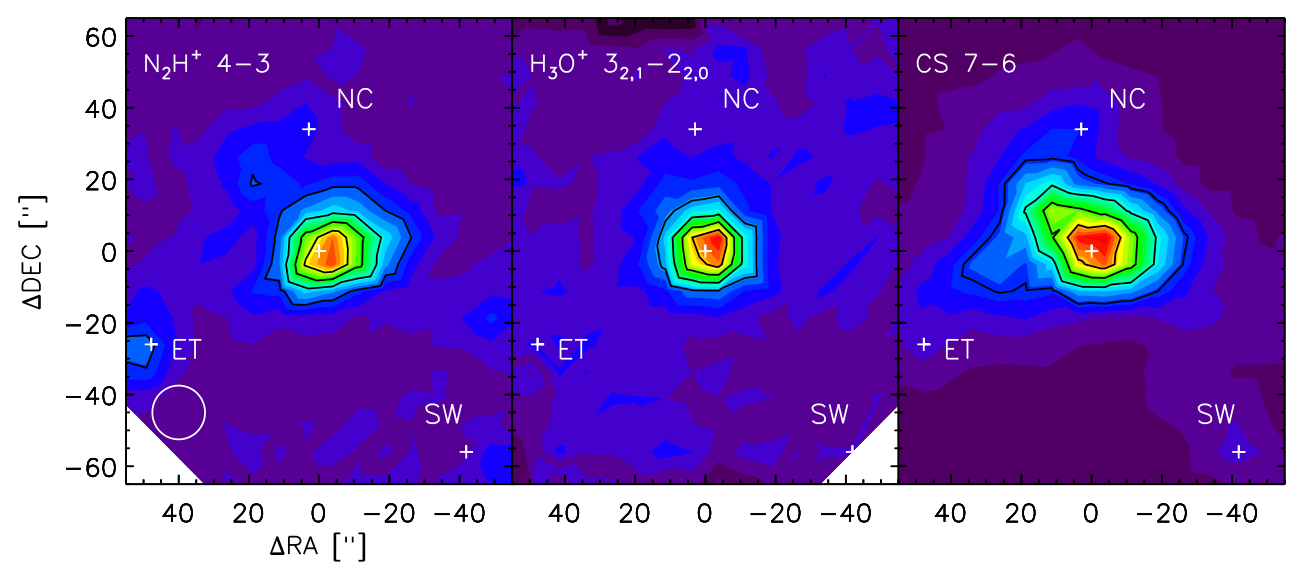

Fig. 6. Spatial distribution of $\mathrm{N}_{2} \mathrm{H}^{+}$and $\mathrm{H}_{3} \mathrm{O}^{+}$ions and CS. The crosses correspond to subregions: source center, eastern tail (ET), northern clump (NC), and southwest clump (SW). The contours correspond to the $20 \%, 40 \%, 60 \%$, and $80 \%$ of the maximum line intensity.

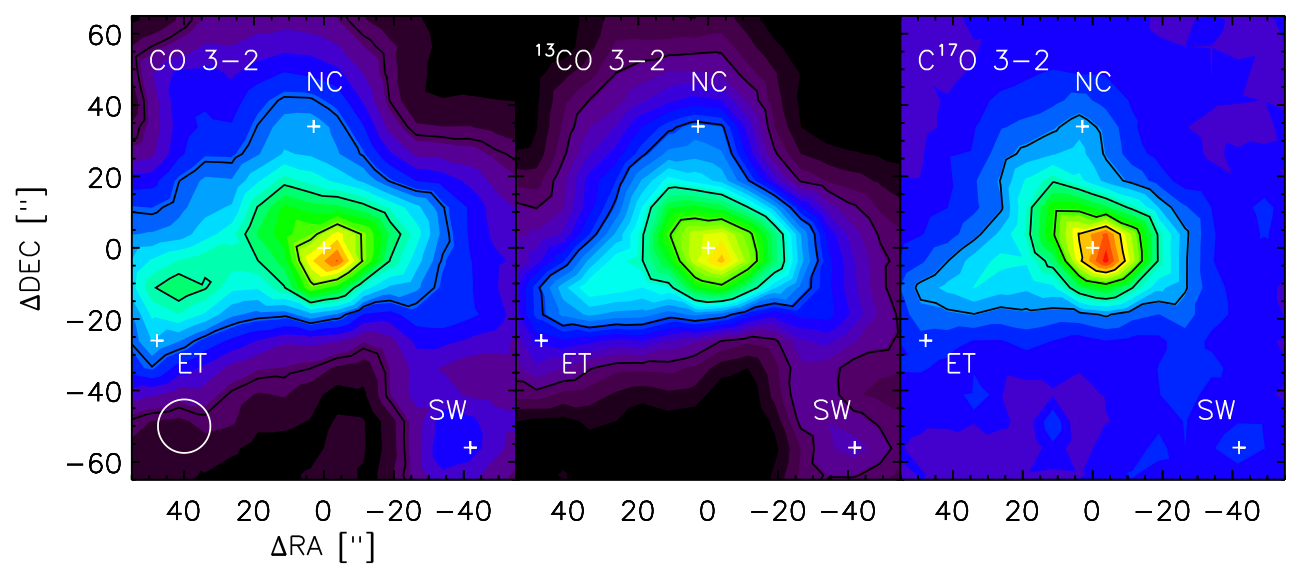

Fig. 7. Spatial distribution of $\mathrm{CO},{ }^{13} \mathrm{CO}$, and $\mathrm{C}^{17} \mathrm{O}$ line emission. The crosses correspond to subregions: source center, eastern tail (ET), northern clump (NC), and southwest clump (SW). The contours correspond to the $20 \%, 40 \%, 60 \%$, and $80 \%$ of the maximum line intensity.

- $\mathrm{H}_{2} \mathrm{CO}$ (formaldehyde) is a commonly used temperature and density tracer in Galactic star-forming regions (e.g., Mangum \& Wootten 1993) and in external galaxies (e.g., Mangum et al. 2008) as well. Previous results from this line survey focused on this molecule, which revealed a large $\sim 3 \times 3 \mathrm{pc}$ region around the W49A center with kinetic temperatures of $>100 \mathrm{~K}$ and densities of $>10^{5} \mathrm{~cm}^{-3}$ (Nagy et al. 2012). Its isotopologue $\mathrm{H}_{2}^{13} \mathrm{CO}$ is also detected toward the center.

- Similar to $\mathrm{H}_{2} \mathrm{CO}, \mathrm{H}_{2} \mathrm{CS}$ (thio-formaldehyde) is a nearprolate rotor, which allows for the estimation of both kinetic temperature and density. Though its spatial extent $\left(30^{\prime \prime} \times 17^{\prime \prime}\right)$ given by a 2D Gaussian fit toward the center is similar to that of $\mathrm{H}_{2} \mathrm{CO}, \mathrm{H}_{2} \mathrm{CS}$ was not detected toward the other main regions (eastern tail, northern clump, and southwest clump). Its isotopologue $\mathrm{H}_{2} \mathrm{C}^{34} \mathrm{~S}$ is also detected.

- We detected a few ions, including $\mathrm{HCO}^{+}$and its isotopologues $\mathrm{HC}^{18} \mathrm{O}^{+}, \mathrm{H}^{13} \mathrm{CO}^{+}$, and $\mathrm{HC}^{17} \mathrm{O}^{+}$. As a dense gas tracer, $\mathrm{HCO}^{+}$data in the SLS survey were analyzed by Roberts et al. (2011). Other detected ions not mentioned in the sections above include $\mathrm{H}_{3} \mathrm{O}^{+}$and $\mathrm{N}_{2} \mathrm{H}^{+} . \mathrm{N}_{2} \mathrm{H}^{+}$shows a larger spatial extent than $\mathrm{H}_{3} \mathrm{O}^{+}$(Fig. 6) covering the eastern tail and northern clump regions. $\mathrm{H}_{3} \mathrm{O}^{+}$is an important chemical ingredient of molecular clouds as it can be related to the formation of water in the gas phase and is formed via $\mathrm{OH}^{+}$and $\mathrm{H}_{2} \mathrm{O}^{+}$ions, which have recently been found to be important tracers of the cosmic-ray ionization rate in the diffuse interstellar medium (e.g., Neufeld et al. 2010; Gerin et al. 2010; Hollenbach et al. 2012). Strong emission lines of $\mathrm{OH}^{+}, \mathrm{H}_{2} \mathrm{O}^{+}$, and $\mathrm{H}_{3} \mathrm{O}^{+}$ions are also found in AGNs (e.g., Van der Werf et al. 2010). Detections of $\mathrm{H}_{3} \mathrm{O}^{+}$transitions toward starburst galaxies and AGNs have also been reported with the JCMT (e.g., Aalto et al. 2011; and Van der Tak et al. 2008).

- CS is a well-known tracer of dense gas that has been analyzed both in Galactic star-forming regions (e.g., Helmich \& van Dishoeck 1997) and in external galaxies, including starburst galaxies (e.g., Aladro et al. 2011b). Multiple rotational transitions of CS have previously been studied in W49A by Serabyn et al. (1993), leading to the idea that the large star formation activity in W49A has been triggered by a cloudcloud collision. CS $J=7-6$ has been detected toward W49A with a spatial extent of $\sim 40^{\prime \prime} \times 24^{\prime \prime}$ (Fig. 6). Its isotopologues ${ }^{13} \mathrm{CS}$ and $\mathrm{C}^{34} \mathrm{~S}$ have also been detected in the SLS survey.

- We detected two hydrogen recombination lines: H26 $\alpha$ at $353.6 \mathrm{GHz}$ and $\mathrm{H} 33 \beta$ at $335.2 \mathrm{GHz}$. Their measured widths of $\sim 37 \mathrm{~km} \mathrm{~s}^{-1}$ and $\sim 31 \mathrm{~km} \mathrm{~s}^{-1}$ are larger than the widths found for molecular lines detected toward the W49A center in the line survey and are similar to those of other $\mathrm{H}$ recombination lines detected toward 


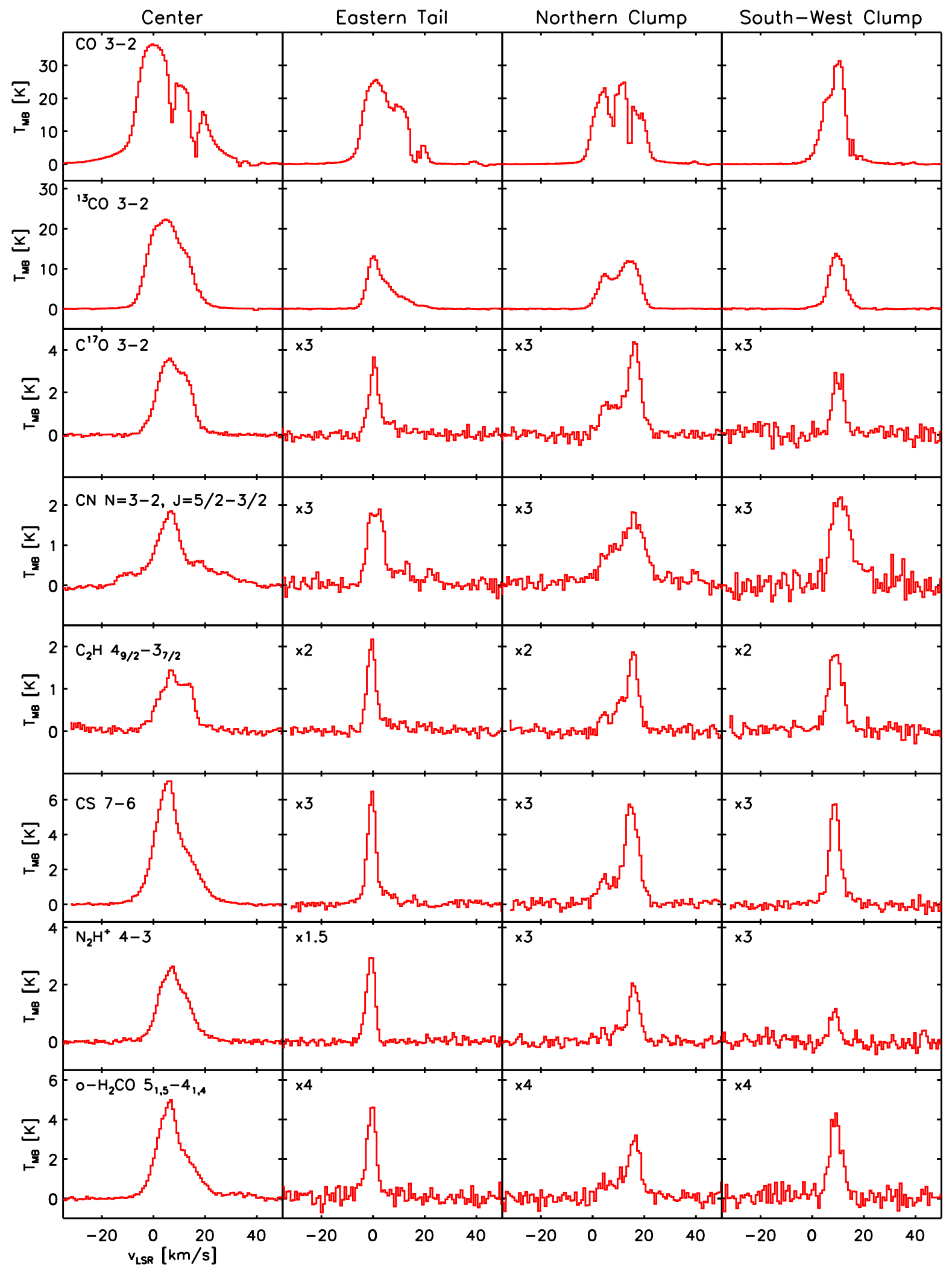

Fig. 8. Line profiles of molecules observed toward the various subregions of W49A.

W49A (e.g., Galvan-Madrid et al. 2013). The velocity of the $\mathrm{H} 26 \alpha$ line is consistent with that of the $\mathrm{H} 41 \alpha$ line detected by Galvan-Madrid et al. (2013). Other hydrogen recombination lines were previously detected in W49A by Gordon \& Walmsley (1990) and Galvan-Madrid et al. (2013).

\subsection{Line profiles and velocity structure}

Figure 8 shows typical line profiles observed toward the various subregions of W49A for the most spatially extended molecular lines, with the exception of $\mathrm{HCN}, \mathrm{HNC}$, and $\mathrm{HCO}^{+}$, which were 


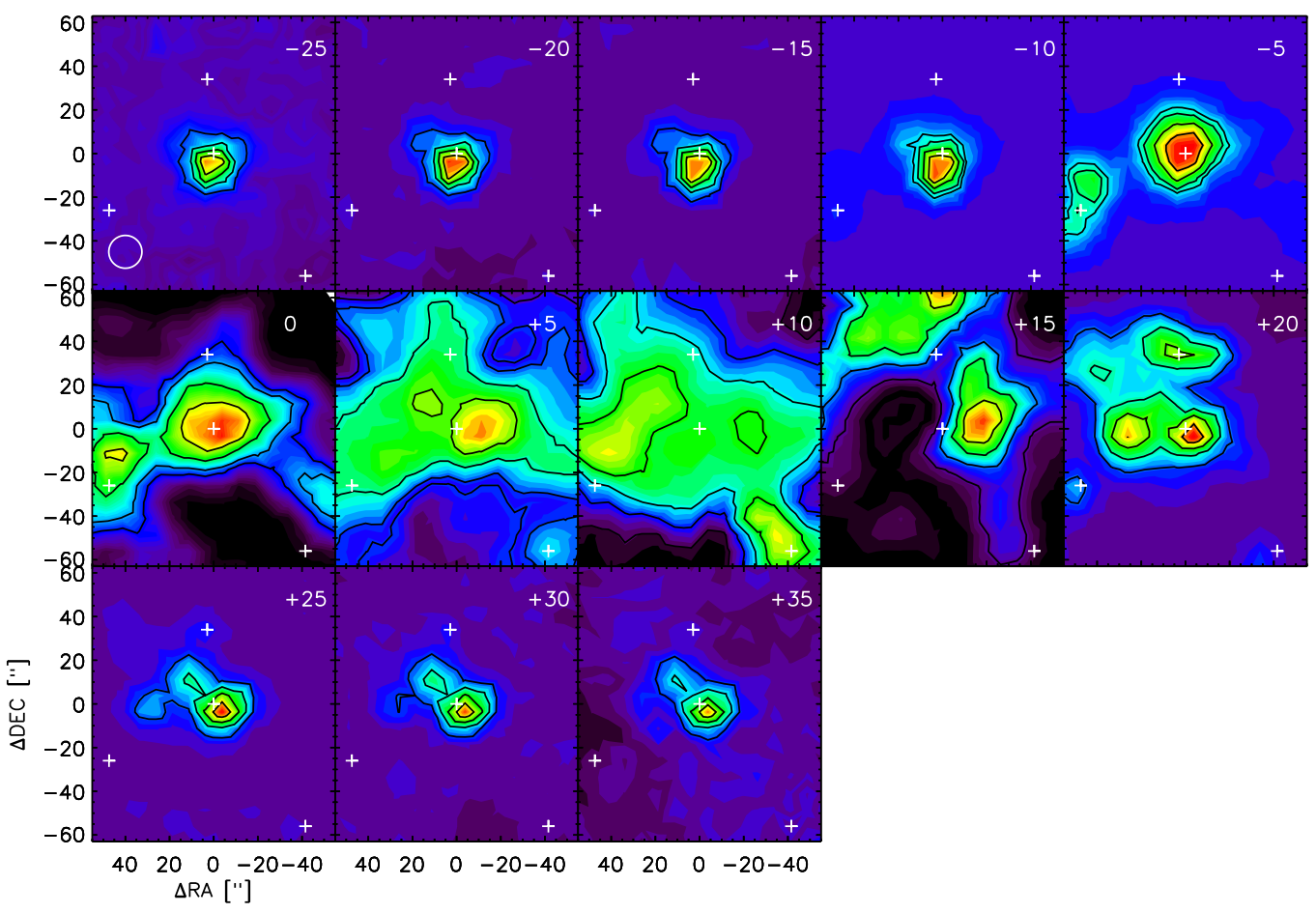

Fig. 9. Channel maps of $\mathrm{CO}$ at a resolution of $\sim 15^{\prime \prime}$ at the given LSR velocities (in $\mathrm{km} \mathrm{s}^{-1}$ ).

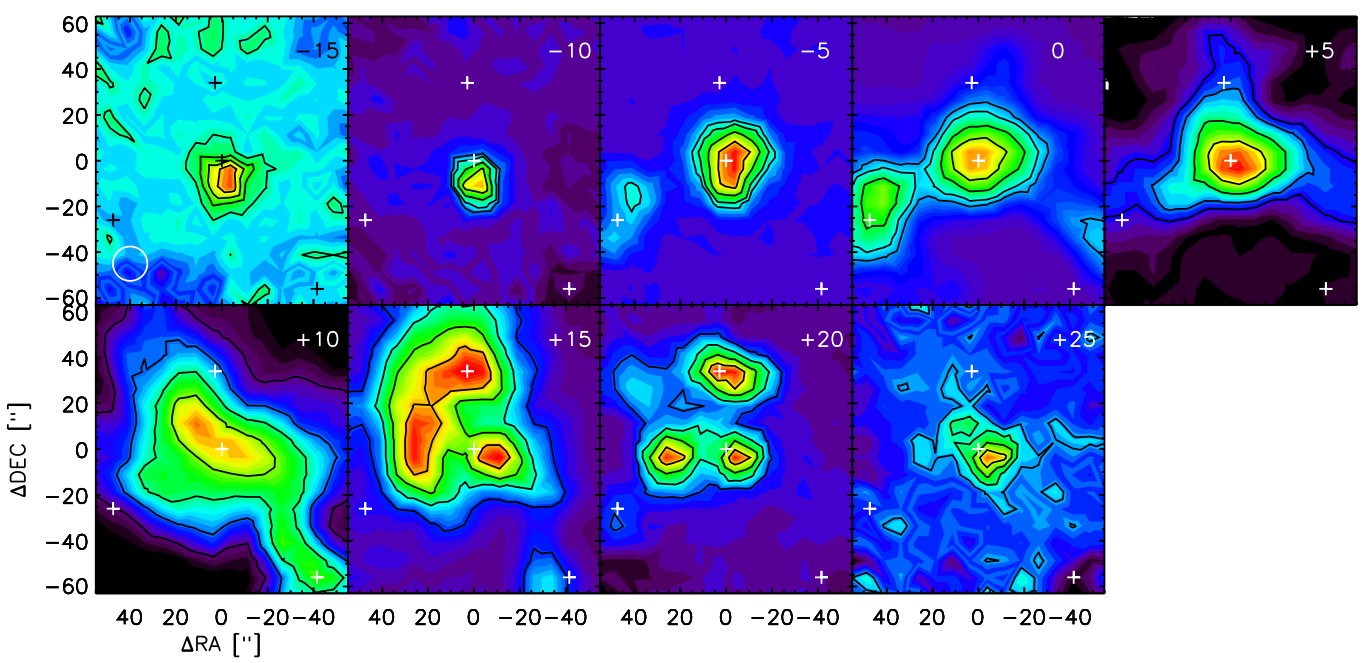

Fig. 10. Channel maps of ${ }^{13} \mathrm{CO}$ at a resolution of $\sim 15^{\prime \prime}$ at the given LSR velocities (in $\mathrm{km} \mathrm{s}^{-1}$ ).

analyzed in Roberts et al. (2011). Most of the detected lines are double-peaked or asymmetric. While lines observed toward the center and the southwest clump peak around the source velocity of $\sim 7-8 \mathrm{~km} \mathrm{~s}^{-1}$ (e.g., Roberts et al. 2011 and references therein), line emission is seen mainly blue-shifted toward the eastern tail, and red-shifted toward the northern clump, similar to what is seen for $\mathrm{HNC}, \mathrm{HCN}$, and $\mathrm{HCO}^{+}$, and their isotopologues (Roberts et al. 2011). Some of the asymmetric, double-peaked line profiles have a brighter blue-shifted than a red-shifted peak, which can be interpreted as gas infalling toward the W49A center. These line profiles, which are consistent with infall signatures, have been observed toward the source center in $\mathrm{HCO}^{+} 1-0$ (Welch et al. 1987, Serabyn et al. 1993) and in the SLS survey traced by $\mathrm{HCN} 4-3$ and 3-2, $\mathrm{HNC}_{4}-3$, and $\mathrm{HCO}^{+} 4-3$ (Roberts et al. 2011). Though most observed lines show an excess emission in the blue-shifted wing of the line profile toward the source center, the line profiles that are most consistent with infall signatures are seen in $\mathrm{HCN} 4-3$ and $3-2$, HNC 4-3, and $\mathrm{HCO}^{+} 4-3$.

To investigate the velocity distribution of the observed line emission, we obtained channel maps for the species shown in Fig. 8. CO 3-2 shows emission in a large range of velocities between -25 and $+35 \mathrm{~km} \mathrm{~s}^{-1}$ (Fig. 9). The most extended line emission is seen around the expected source velocity, in the range between +5 and $+10 \mathrm{~km} \mathrm{~s}^{-1}$. Toward the eastern tail, significant blue-shifted line emission is seen at velocities between -5 and $0 \mathrm{~km} \mathrm{~s}^{-1}$. The northern clump is more prominent at velocities of $\sim 20 \mathrm{~km} \mathrm{~s}^{-1}$, but also shows significant emission around the source velocity. The southwest clump is only seen around the expected source velocity (between 5 and $10 \mathrm{~km} \mathrm{~s}^{-1}$ ). Significant line emission is seen at a velocity of $+15 \mathrm{~km} \mathrm{~s}^{-1}$, which does not correspond to any of the analyzed subregions, including the center. The ${ }^{13} \mathrm{CO} 3-2$ line shows a very similar structure (Fig. 10) to that traced by $\mathrm{CO} 3-2$ with the main difference 


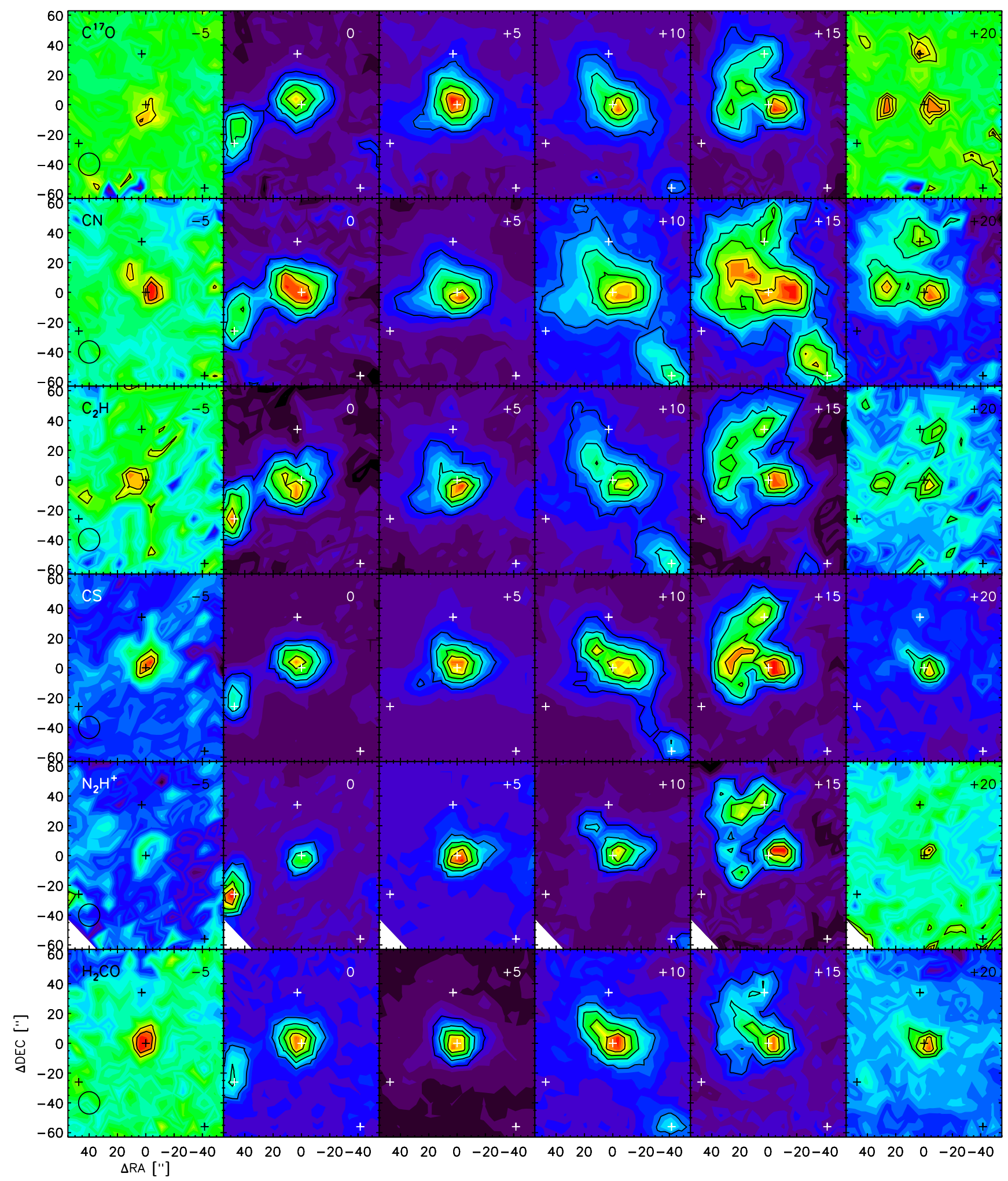

Fig. 11. Channel maps of the most spatially extended species in the SLS at the given LSR velocities (in $\mathrm{km} \mathrm{s}^{-1}$ ).

at a velocity of $+15 \mathrm{~km} \mathrm{~s}^{-1}$, likely related to a self-absorption of the optically thick CO 3-2 lines around the center.

Lines that show spatially extended emission other than $\mathrm{CO}$ and ${ }^{13} \mathrm{CO}$ are distributed over the velocity range between -5 and $+20 \mathrm{~km} \mathrm{~s}^{-1}$ (Fig. 11). The strongest emission is seen in $\mathrm{CN}$, especially the red-shifted wings of the $\mathrm{CN}$ lines. While other molecules show emission toward the center and the northern clump at a velocity of $+15 \mathrm{~km} \mathrm{~s}^{-1}, \mathrm{CN}$ emission at this velocity is also detected toward the southwest clump.

\subsection{Excitation and column densities}

Table 2 includes a number of species with at least three detected transitions covering a sufficient energy range to probe 

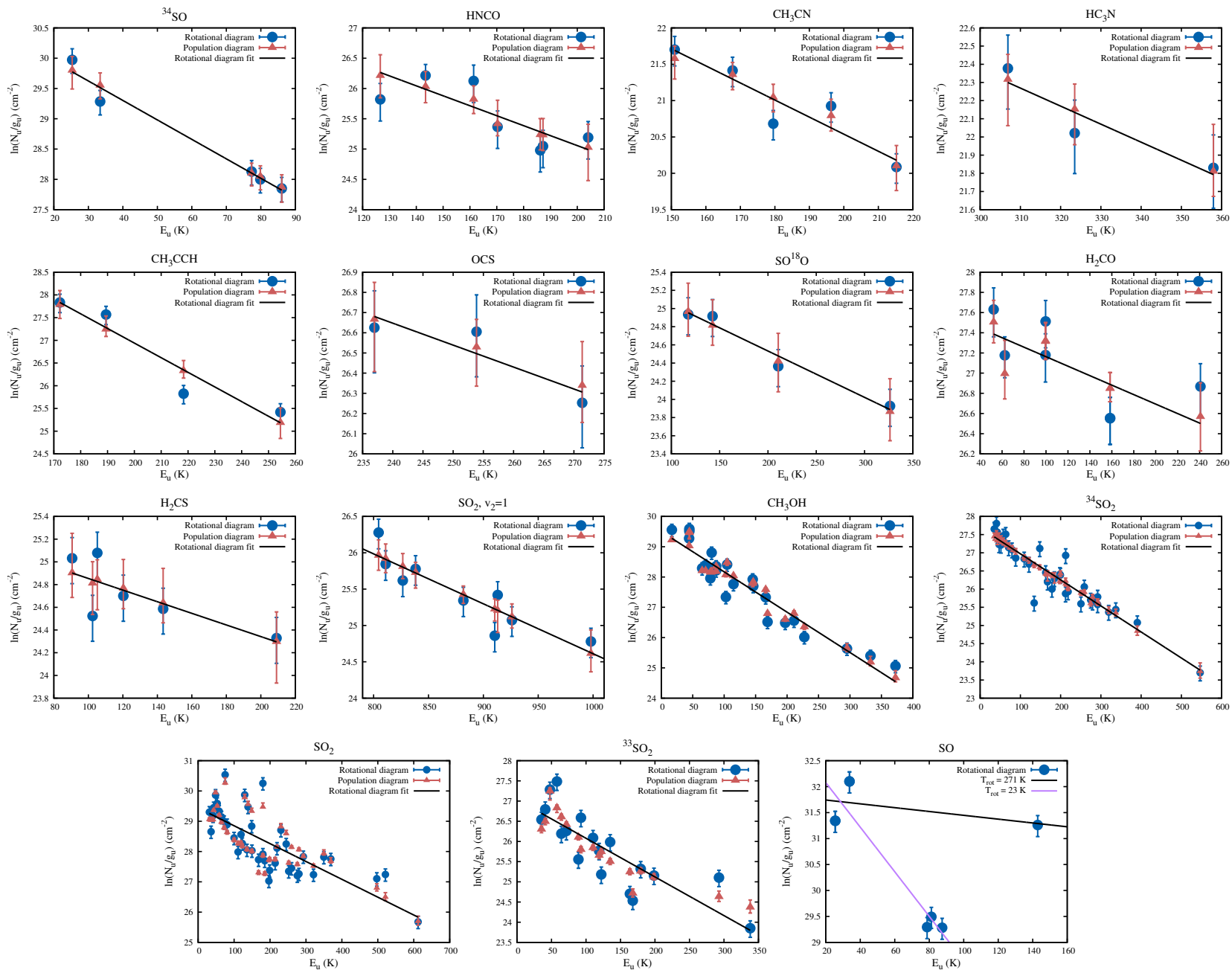

Fig. 12. Rotation and population diagram plots toward the center. For $\mathrm{CH}_{3} \mathrm{OH}, \mathrm{H}_{2} \mathrm{CO}$, and $\mathrm{SO}_{2}$, the transitions from the high-frequency extension of the SLS (between 360-373 GHz) were originally analyzed in Nagy et al. (2012).

excitation conditions. We estimate the excitation conditions of these molecules using the population diagram method introduced by Goldsmith \& Langer (1999). Column densities $\left(N_{\mathrm{tot}, 1}\right)$ and excitation temperatures $\left(T_{\mathrm{ex}}\right)$ can be estimated based on

$\ln \left(\frac{N_{\mathrm{u}}}{g_{\mathrm{u}}}\right)=\ln \left(\frac{N_{\mathrm{tot}, 1}}{Q_{\mathrm{rot}}}\right)-\frac{E_{\mathrm{u}}}{k T_{\mathrm{ex}}}+\ln \left(\frac{\Omega_{\mathrm{s}}}{\Omega_{\mathrm{a}}}\right)-\ln \left(C_{\tau}\right)$,

where $N_{\mathrm{u}}$ is the observed upper state column density of the molecule including line opacity and beam-source coupling effects; $g_{\mathrm{u}}$ is the degeneracy of the upper state; $Q_{\mathrm{rot}}$ is the rotational partition function; $k$ is the Boltzmann constant; $E_{\mathrm{u}}$ is the upper level energy; $f=\Omega_{\mathrm{s}} / \Omega_{\mathrm{a}}$ is the source filling factor, with $\Omega_{\mathrm{a}} \sim 15^{\prime \prime}$ the beam size and $\Omega_{\mathrm{s}}$ the source size; and $C_{\tau}=\tau /\left(1-\mathrm{e}^{-\tau}\right)$, where $\tau$ is the optical depth. For a uniform beam filling $\left(\Omega_{\mathrm{a}} \sim \Omega_{\mathrm{s}}\right)$ and low optical depth, Eq. (1) reduces to a rotational diagram with a rotational temperature $T_{\text {rot }}$ and total column density $N_{\text {tot }}$. For a rotational diagram Eq. (1) is simplified to only the first two terms on the right side of the equation. The terms containing the correction for the opacity and the beam filling factor are not included.

We evaluate Eq. (1) for a set of $N_{\text {tot, }, 1}, T_{\mathrm{ex}}, f$, and $C_{\tau}\left(N_{\mathrm{tot}, 1}\right.$, $T_{\text {ex }}$ ). We apply $\Omega_{\mathrm{s}}$ in the range between $1^{\prime \prime}$ and $15^{\prime \prime}$ (uniform beam filling) and a column density in the range between $10^{12} \mathrm{~cm}^{-2}$ and $10^{18} \mathrm{~cm}^{-2}$. For molecules with just three detected transitions we use $T_{\text {rot }}=T_{\text {ex }}$ to derive a best-fit source size and column density.

The results are summarized in Table 2 . The rotation- and population diagrams are shown on Fig. 12. The $\ln \left(N_{\mathrm{u}} / g_{\mathrm{u}}\right)$ values corresponding to the rotational and the population diagrams are close to each other, as many of the observed lines are optically thin (with a few exceptions of some of the $\mathrm{SO}_{2}, \mathrm{CH}_{3} \mathrm{OH}$, $\mathrm{H}_{2} \mathrm{CO}$, and ${ }^{33} \mathrm{SO}_{2}$ lines). The scatter in the population diagram values is smaller than in the rotational diagrams as we corrected the optically thicker lines for their optical depth. In addition, we corrected the lines for the best-fit size of the emitting region.

Most molecular lines have excitation temperatures between $\sim 100$ and $\sim 200 \mathrm{~K}$, with $\mathrm{H}_{2} \mathrm{CO}, \mathrm{H}_{2} \mathrm{CS}$, and $\mathrm{SO}^{18} \mathrm{O}$ showing the highest excitation. The population diagrams suggest a nonuniform beam filling in several cases and result in emission originating in regions of $\sim 1-4^{\prime \prime}$, which are consistent with the sizes of hot cores and UCHII regions revealed by high resolution studies (e.g., Wilner et al. 2001). Together with the spatially extended emission seen in the maps, this is evidence that the gas in W49A has a clumpy structure.

$\mathrm{CH}_{3} \mathrm{CCH}$ has a very low excitation temperature toward the center $(\sim 32 \mathrm{~K})$, suggesting an origin in colder material around the central cluster of UC HII regions and hot cores. $\mathrm{CH}_{3} \mathrm{CCH}$ was also found to originate in a lower excitation component toward Sgr B2, Orion and DR 21 (Churchwell \& Hollis 1983). 
Table 3. Column densities toward the four analyzed subregions of W49A (in case of detections) derived in the LTE optically thin approximation.

\begin{tabular}{|c|c|c|c|c|c|}
\hline \multirow[t]{2}{*}{ Species } & \multirow[t]{2}{*}{ Transition } & \multicolumn{4}{|c|}{ Column density $\left(\mathrm{cm}^{-2}\right)$} \\
\hline & & Center & Eastern tail & Northern clump & Southwest clump \\
\hline $\mathrm{C}_{2} \mathrm{H}$ & $4_{9 / 2}-3_{7 / 2}$ & $(1.7-2.8) \times 10^{15}$ & $(3.8-6.0) \times 10^{14}$ & $(5.7-9.0) \times 10^{14}$ & $(5.2-8.3) \times 10^{14}$ \\
\hline NO & $7 / 2-5 / 2^{+}$ & $(1.0-1.8) \times 10^{17}$ & $(0.8-1.4) \times 10^{16}$ & $(1.0-1.9) \times 10^{16}$ & $(4.8-8.8) \times 10^{15}$ \\
\hline $\mathrm{H}^{13} \mathrm{CN}$ & $4-3$ & $(1.0-1.4) \times 10^{14}$ & $(6.5-9.8) \times 10^{11}$ & $(2.0-3.0) \times 10^{12}$ & $(1.6-2.5) \times 10^{12}$ \\
\hline $\mathrm{H}^{13} \mathrm{CO}^{+}$ & $4-3$ & $(2.5-3.8) \times 10^{13}$ & $(0.8-1.3) \times 10^{12}$ & $(1.3-2.0) \times 10^{12}$ & $(1.3-1.9) \times 10^{12}$ \\
\hline $\mathrm{CN}$ & $N=3-2, J=5 / 2-3 / 2$ & $(1.4-2.2) \times 10^{15}$ & $(3.0-4.8) \times 10^{14}$ & $(4.3-6.9) \times 10^{14}$ & $(3.7-5.9) \times 10^{14}$ \\
\hline $\mathrm{N}_{2} \mathrm{H}^{+}$ & $4-3$ & $(3.0-4.4) \times 10^{13}$ & $(0.7-1.1) \times 10^{13}$ & $(4.1-6.0) \times 10^{12}$ & $(1.4-2.0) \times 10^{12}$ \\
\hline $\mathrm{CS}$ & $7-6$ & $(4.4-5.6) \times 10^{14}$ & $(4.7-6.1) \times 10^{13}$ & $(7.5-9.6) \times 10^{13}$ & $(4.8-6.2) \times 10^{13}$ \\
\hline$C^{34} S$ & $7-6$ & $(6.2-8.8) \times 10^{13}$ & $(2.2-2.8) \times 10^{12 a}$ & $(4.2-6.1) \times 10^{12}$ & $(5.8-8.3) \times 10^{11}$ \\
\hline $\mathrm{H}_{2} \mathrm{~S}$ & $3_{2,1}-3_{1,2}$ & $4.9 \times 10^{15}$ & & & \\
\hline $\mathrm{HCO}$ & $4_{1,4}-3_{1,3}$ & $(1.4-2.4) \times 10^{15}$ & & & \\
\hline $\mathrm{CO}^{+}$ & $N=3-2, F=5 / 2-3 / 2$ & $(1.4-2.2) \times 10^{13}$ & & & \\
\hline $\mathrm{HCS}^{+}$ & $8-7$ & $(1.8-2.2) \times 10^{13}$ & & & \\
\hline $\mathrm{SO}^{+}$ & $8-7$ & $(4.4-5.7) \times 10^{14}$ & & & \\
\hline $\mathrm{H}_{3} \mathrm{O}^{+}$ & $3_{2,1}-2_{2,0}$ & $(4.8-5.3) \times 10^{14}$ & & & \\
\hline $\mathrm{SiO}$ & $8-7$ & $(0.8-1.0) \times 10^{14}$ & & & \\
\hline
\end{tabular}

Notes. The two values correspond to adopted temperatures of $75 \mathrm{~K}$ and $150 \mathrm{~K}$, respectively. The error of these column densities is about a factor of 2 dominated by the uncertainty in $T_{\mathrm{ex}} \cdot{ }^{\left({ }^{a}\right)}$ Based on $N(\mathrm{CS})$ and an isotopic ratio of ${ }^{32} \mathrm{~S} /{ }^{34} \mathrm{~S}$ of 22 (Frerking et al. 1980).

The other species that shows a very low excitation temperature is ${ }^{34} \mathrm{SO}\left(T_{\mathrm{ex}} \sim 33 \mathrm{~K}\right)$. Results for SO are not listed in Table 2 as the detected SO transitions are not consistent with a single excitation temperature. This may suggest that some of the detected SO lines originate in the low-excitation component suggested by the ${ }^{34}$ SO lines, and the others from the high-excitation $(\gtrsim 100 \mathrm{~K})$ component in which most detected molecular lines originate. Apart from different excitation conditions, gas components with different source sizes (beam-filling factors) may contribute to the observed line emission, as suggested by the large range of source sizes corresponding to the different sulphur-bearing molecules. Distinguishing between these explanations requires observations of more SO transitions.

Differences in the excitation of the subregions (northern clump, eastern tail, and southwest clump) are well traced by $\mathrm{H}_{2} \mathrm{CO}$, as we detected more of its transitions with a spatial extent large enough to include the discussed subregions. More details are shown in Nagy et al. (2012).

For molecules with no information available on their excitation we derive a column density in the optically thin LTE approximation, assuming rotation temperatures in the range between $75 \mathrm{~K}$ and $150 \mathrm{~K}$, as most molecules with multiple detected transitions have rotation temperatures that fall into this range. Then we derive column densities using

$N_{\text {tot }}=\frac{8 \pi k v^{2}}{h c^{3}} \frac{Q\left(T_{\text {rot }}\right)}{g_{\mathrm{u}} A_{\mathrm{ul}}} \mathrm{e}^{E_{\mathrm{u}} / k T_{\text {rot }}} \int T_{\mathrm{mb}} \mathrm{d} v$.

Table 3 includes a selection of molecules that have been detected in AGNs, starburst galaxies, and in Galactic star-forming regions. We obtained the line intensities using a Gaussian fit for nearly Gaussian line profiles. For double-peaked or asymmetric line profiles, we used the velocity-integrated intensity to calculate column densities. The error of these column densities is about a factor of 2 , dominated by the uncertainty in $T_{\mathrm{ex}}$. For the species where the number of detected transitions was not high enough to correct for the opacity, the optically thin approximation results in an underestimate of the column densities. This is the case for some of the species detected toward the center, such as for $\mathrm{CS}$ and $\mathrm{CN}$. The CS column density is underestimated by a factor of $\sim 3$, by applying the observed $N\left(\mathrm{C}^{34} \mathrm{~S}\right)$ and an isotopic ratio of ${ }^{32} \mathrm{~S} /{ }^{34} \mathrm{~S}$ of 22 (Frerking et al. 1980). The column density of $\mathrm{CN}$ cannot be constrained as no isotopologue of $\mathrm{CN}$ was detected in the SLS survey. Therefore, the value derived from CN is a lower limit of the $\mathrm{CN}$ column density. We discuss these column densities in more details in Sect. 4.

\section{Discussion}

The data reveal a complex structure both in terms of kinematics and chemical composition. The molecular line tracers detected in the SLS frequency range can be related to various physical components, including shocked regions and PDRs. Some of the detected molecules can be used for a comparison between the local starburst seen in W49A and global starburst phenomenon seen for some external galaxies. In the next sections, we follow up on the simple classification of the detected species presented in Sect. 3.2 based on kinematics and a comparison to wellknown environments of UV-dominated and shock chemistry.

\subsection{The kinematics of chemically related species}

In Table A.1 we list the basic line parameters derived from fitting a single profile Gaussian to each line. Here we use the velocities and FWHM line widths to obtain information on the kinematics of the previously discussed species. This is a simplification, as the fitting of most line profiles requires more components than a single Gaussian fit (e.g., Galvan-Madrid et al. 2013; Roberts et al. 2011). In this simple comparison fitting the line profiles accurately is beyond our scope; we provide a comparison of the line widths and velocities of the observed lines, which gives information on the regions where the bulk of emission arises from. For the most asymmetric lines we use only the line width derived using a Gaussian fit, and for its $v_{\text {LSR }}$ we use the velocity of the peak intensity. Most of the observed lines correspond to species associated with shock tracers (as explained in Sect. 3.2.1) including sulphur-bearing molecules. Figure 14 includes a summary of the fitted FWHM and peak velocity values observed for the molecules associated with shock tracers. The lines of molecules with a possible origin in shocks covers a large range of line widths $\left(9-19 \mathrm{~km} \mathrm{~s}^{-1}\right)$ and peak velocities $\left(7-14 \mathrm{~km} \mathrm{~s}^{-1}\right)$, but most species peak at a velocity of $9-10 \mathrm{~km} \mathrm{~s}^{-1}$ and have a width of $15-16 \mathrm{~km} \mathrm{~s}^{-1}$. The large range in the observed parameters is possibly related to the large region $(\sim 0.8 \mathrm{pc})$ covered by 


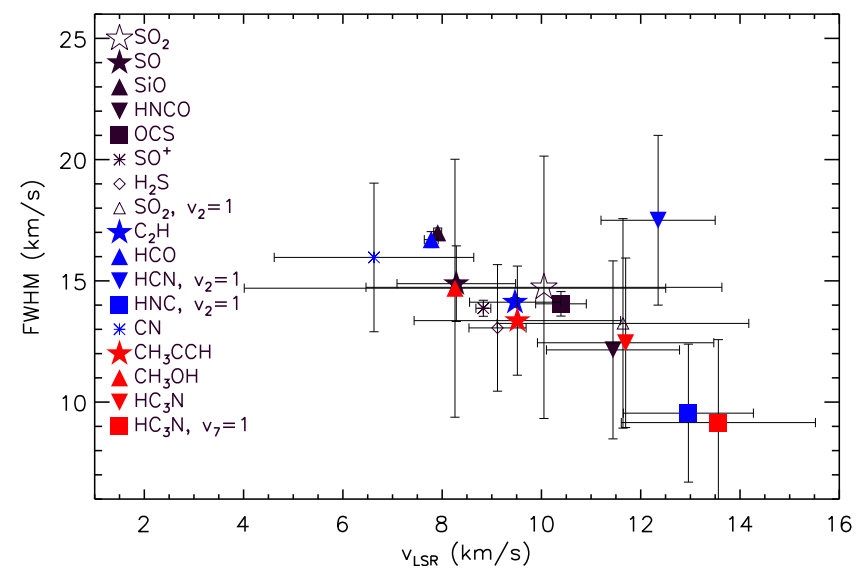

Fig. 13. Measured line widths vs. peak velocities of species detected toward the W49A center. The molecules classified as shock tracers are shown in black, the molecules classified as PDR tracers are shown in blue, and the molecules classified as complex organic molecules are shown in red. Molecules with double-peaked line profiles are not included in this plot.

the JCMT beam in the $345 \mathrm{GHz}$ band, which covers multiple sources that are only fully resolved with interferometers, such as in the maps presented in Galvan-Madrid et al. (2013). Figure 13 shows the fitted peak velocities vs the line widths for species corresponding to shock tracers (black color), PDR tracers (blue), and complex organic molecules (red). The error bars represent the range of the parameters for the different transitions. All three groups cover a substantial fraction of the parameter space for both velocities and line widths that we discuss. The highest velocities are found for the vibrationally excited molecules. For $\mathrm{HCN}, v_{2}=1$, the high peak velocities are combined with large line widths $\left(F W H M \sim 20 \mathrm{~km} \mathrm{~s}^{-1}\right)$. The fact that the molecules that trace different chemistries do not correlate with kinematics may be related to the fact that different individual sources are covered by the JCMT beam. The kinematical signatures are likely to be dependent on the source that they correspond to, and not on the different chemical groups to which they can be related.

Another method to test whether the assumed chemically related species indeed trace different regions is a comparison of the distributions of the observed line parameters. As most observed transitions correspond to shock tracers, we compare the distribution of the observed $v_{\text {LSR }}$ and FWHM line widths to those of every other transition. Figure 14 shows the $v_{\text {LSR }}$ and FWHM line width distributions of the species classified as shock tracers and of all the other transitions. We compare the distribution of the fitted parameters of these groups of species using Kolmogorov-Smirnov tests, as shown in Fig. 14. The probability that the two distributions are different is about $20 \%$ for the line widths and about $38 \%$ for the peak velocities. This supports our conclusion that the classification of chemically related species is not confirmed by the measured line parameters, likely, because on the scale probed in this paper they correspond to many different sources, which are not resolved by the JCMT beam. The larger difference seen in the velocity distributions compared to the distributions of the line widths is likely due to the slightly asymmetric line profiles included in the samples.

\subsection{The importance of UV irradiation}

We probe the importance of UV irradiation in the chemistry of W49A with a comparison of the observed column density ratios
Table 4. Summary of observed column density ratios in W49A (center, eastern tail, northern clump and southwest clump, when available) and in Galactic PDRs.

\begin{tabular}{lccccc}
\hline \hline Ratio & W49A & $\begin{array}{c}\text { Orion } \\
\text { Bar }\end{array}$ & $\begin{array}{c}\text { NGC } \\
7023\end{array}$ & $\begin{array}{c}\text { Mon } \\
\text { R2 }\end{array}$ & $\begin{array}{c}\text { Horse- } \\
\text { head }\end{array}$ \\
\hline $\mathrm{CN} / \mathrm{HCN}$ center & 0.55 & $3.0^{a}$ & $4.5^{b}$ & $2-12^{c}$ & \\
$\mathrm{CN} / \mathrm{HCN} \mathrm{ET}$ & $3.8-6.9$ & & & & \\
$\mathrm{CN} / \mathrm{HCN} \mathrm{NC}$ & $2-4.6$ & & & & \\
$\mathrm{CN} / \mathrm{HCN} \mathrm{SWC}$ & $0.4-0.95$ & & & & \\
$\mathrm{SiO} / \mathrm{H}^{13} \mathrm{CO}^{+}$ & $2.7-3.3$ & $3-14^{d}$ & $0.54^{d}$ & $0.03-0.37^{e}$ & \\
$\mathrm{HCO} / \mathrm{SiO}$ & $16-23$ & $2-9^{d}$ & $>4.6-10^{d}$ & $50-720^{e}$ & \\
$\mathrm{CO}^{+} / \mathrm{H}^{13} \mathrm{CO}^{+}$ & $<0.56$ & $0.52^{a, f}$ & $0.6^{b, f}$ & $0.53^{c}$ & \\
$\mathrm{CS}^{-} \mathrm{HCS}^{+}$ & $76-108$ & & & $11-25^{c}$ & $175^{g}$ \\
$\mathrm{SO}^{+} / \mathrm{SO}_{2}$ & $<0.02$ & $0.4-1^{f}$ & $>0.4^{f}$ & $0.2-0.7^{c}$ & \\
\hline
\end{tabular}

References. (a) Fuente et al. (1996); (b) Fuente et al. (1993); (c) Ginard et al. (2012); (d) Schilke et al. (2001); (e) Rizzo et al. (2005); (f) Fuente et al. (2003); (g) Goicoechea et al. (2006).

to values observed toward well-known Galactic PDRs, and by a comparison to predictions of PDR models.

For the comparison of column density ratios to those observed toward PDRs, we select a sample of lines that have been observed both in the SLS survey and in multiple PDRs (Table 4). One of the most commonly used tracers of PDRs is the $[\mathrm{CN}] /[\mathrm{HCN}]$ abundance ratio, which was found to be larger than unity in NGC 7023 (Fuente et al. 1993). An increase in the abundance ratio of $[\mathrm{CN}] /[\mathrm{HCN}]$ in PDRs is a result of the production of $\mathrm{CN}$ via the photodissociation of $\mathrm{HCN}$. Using the $\mathrm{HCN}$ column densities presented in (Nagy et al. 2012) derived in a nonLTE approximation and CN column densities from Table 2, we estimate a $[\mathrm{CN}] /[\mathrm{HCN}]$ abundance ratio of $\sim 2.0-4.6$ toward the northern clump, $\sim 3.8-6.9$ toward the eastern tail, $\sim 0.4-0.95$ toward the southwest clump, and $[\mathrm{CN}] /[\mathrm{HCN}]=0.04-0.07$ toward the center. The low $[\mathrm{CN}] /[\mathrm{HCN}]$ ratio toward the center suggests that the chemistry is only partially affected by UV-irradiation.

Though $\mathrm{SiO}$ is a tracer of shocks, it has been detected toward several PDRs as well (e.g., Schilke et al. 2001; Rizzo et al. 2005). Typical $[\mathrm{SiO}] /\left[\mathrm{H}^{13} \mathrm{CO}^{+}\right]$abundance ratios of $0.03-0.37$ were found toward Mon R2 (Rizzo et al. 2005), 0.54 toward NGC 7023 (Schilke et al. 2001), and 3-14 toward the Orion Bar (Schilke et al. 2001), based on detections toward multiple positions. We measure an $[\mathrm{SiO}] /\left[\mathrm{H}^{13} \mathrm{CO}^{+}\right]$abundance ratio of 2.7-3.3 toward the W49A center. The HCO abundance with respect to the $\mathrm{SiO}$ abundance was found to be 50-720 in Mon R2 (Rizzo et al. 2005), while it is in the range between 16 and 23 toward the W49A center. The $[\mathrm{HCO}] /[\mathrm{SiO}]$ abundance ratio of >4.6-10 measured toward NGC 7023 (Schilke et al. 2001 ) is based on an upper limit for the $\mathrm{SiO}$ column density. The $[\mathrm{HCO}] /[\mathrm{SiO}]$ abundance ratio toward the Orion Bar was measured to be $2-9$, based on detections toward multiple positions. Though the large $\left(\sim 17 \mathrm{~km} \mathrm{~s}^{-1}\right)$ line width of SiO detected toward the W49A center (Table A.1) suggests an origin in shocks rather than PDRs, the $[\mathrm{HCO}] /[\mathrm{SiO}]$ and $[\mathrm{SiO}] /\left[\mathrm{H}^{13} \mathrm{CO}^{+}\right]$abundance ratios do not confirm it.

The $\mathrm{CO}^{+}$ion is a tracer of warm $\left(T_{\text {gas }} \gtrsim 500 \mathrm{~K}\right)$ PDR surfaces, corresponding to the region of $A_{V}<2$ mag (Fuente et al. 2003; Rizzo et al. 2003). We measure an abundance ratio of $\left[\mathrm{CO}^{+}\right] /\left[\mathrm{H}^{13} \mathrm{CO}^{+}\right]$of $0.54-0.56$. This is an upper limit on the $\left[\mathrm{CO}^{+}\right] /\left[\mathrm{H}^{13} \mathrm{CO}^{+}\right]$ratio as the $\mathrm{CO}^{+}$line may be blended with $\mathrm{SO}^{17} \mathrm{O}$. Ginard et al. (2012) measure $\left[\mathrm{CO}^{+}\right] /\left[\mathrm{H}^{13} \mathrm{CO}^{+}\right]=0.53$ in Mon R2, similar to that in the Orion $\operatorname{Bar}(0.52$, based on Fuente et al. 1996, 2003) and to that in NGC $7023(0.6$, based on Fuente et al. 1993, 2003). The detection of $\mathrm{CO}^{+}$at several positions 

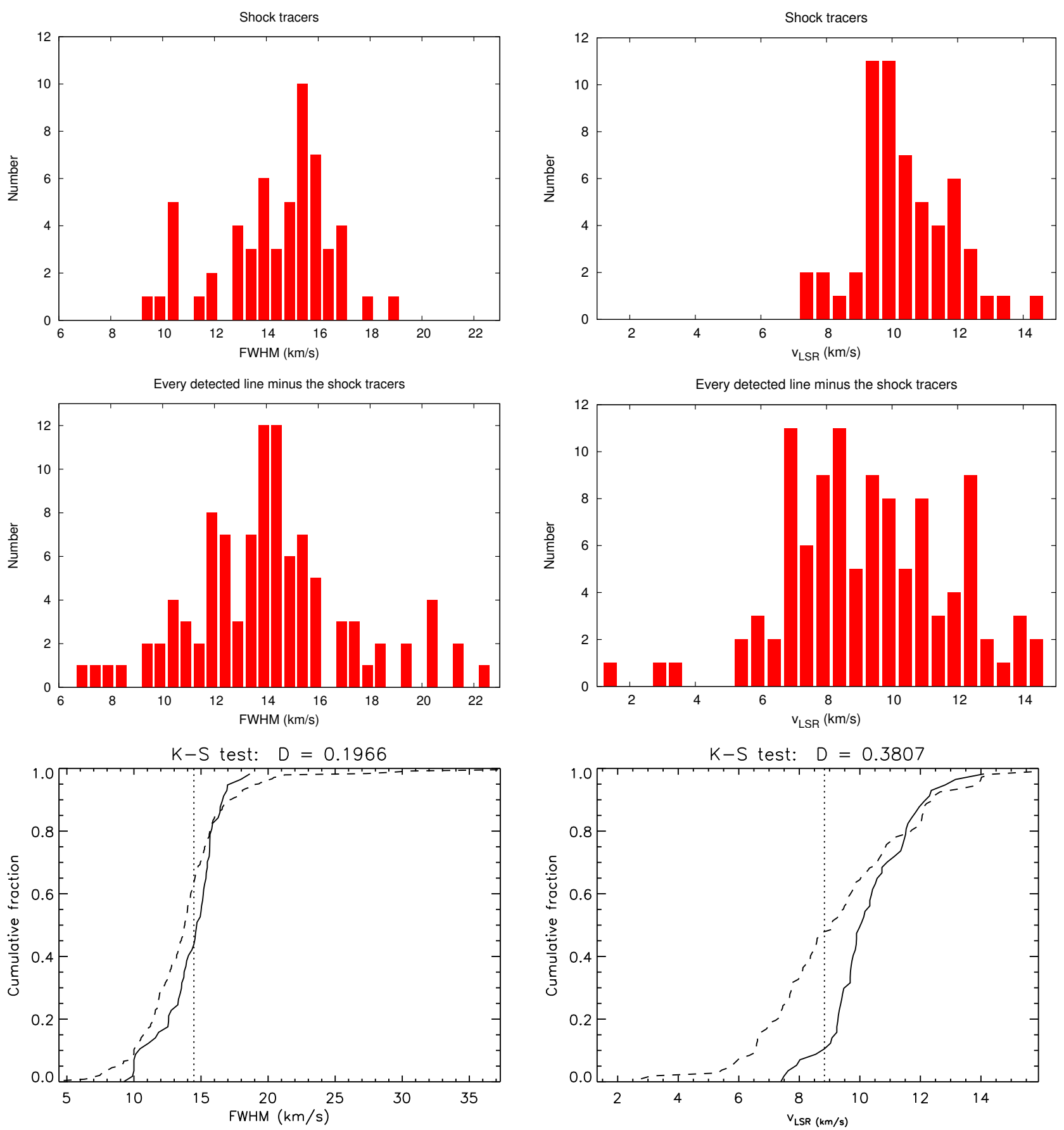

Fig. 14. Top: distribution of the fitted line widths (left) and peak velocities (right) of the lines corresponding to molecules classified as "shock tracers". Middle: distribution of the fitted line widths (left) and peak velocities (right) of every detected line in the line survey, except for the double-peaked and very asymmetric line profiles and the shock tracers shown above. Bottom: K-S test for the line width (left) and peak velocity (right) distributions shown above, corresponding to shock tracers only and to every other molecule that is not classified as a shock tracer. The parameter $D$ is the maximum distance between the cumulative fraction of the shock tracers and the whole sample of lines and is related to the probability of a difference between the distributions. The part of the vertical dotted lines between the two distributions is equivalent to the $D$ parameter.

toward the center suggests the existence of a hot gas component directly exposed to UV irradiation by a radiation field of $G_{0} \sim 3 \times 10^{5}$ (Vastel et al. 2001).

$\mathrm{HCS}^{+}$was found to be abundant in PDRs (Ginard et al. 2012). We measure a CS abundance with respect to that of $\mathrm{HCS}^{+}$of $\sim 76-108$. This is between the lower ratios of 11 and 25 found toward the ionization front and MP2 positions in Mon R2 (Ginard et al. 2012) and the higher $[\mathrm{CS}] /\left[\mathrm{HCS}^{+}\right]$ratio of 175 measured toward the Horsehead nebula (Goicoechea et al. 2006).
This suggests that the $[\mathrm{CS}] /\left[\mathrm{HCS}^{+}\right]$ratio is not a good tracer to probe the contribution of UV irradiation in the chemistry of W49A.

The sulphur-bearing species $\mathrm{SO}^{+}$and $\mathrm{SO}_{2}$ can be related to shock chemistry, but have also been detected in several PDRs. In PDRs $\mathrm{SO}^{+}$is formed near the PDR surface (at $A_{V}<2$ ) through the reaction $\mathrm{S}^{+}+\mathrm{OH} \rightarrow \mathrm{SO}^{+}+\mathrm{H}$ and destroyed by dissociative recombination into $\mathrm{S}$ and $\mathrm{O}$. $\mathrm{SO}$ and $\mathrm{SO}_{2}$ form in the more shielded regions in the PDR. The $\left[\mathrm{SO}^{+}\right] /\left[\mathrm{SO}_{2}\right]$ abundance 
Table 5. Comparison of the column density ratios measured toward the center of W49A to PDR models with pressures of $10^{7} \mathrm{~cm}^{-3} \mathrm{~K}$ and $10^{8} \mathrm{~cm}^{-3} \mathrm{~K}$ illuminated by a radiation field of $\chi=3.5 \times 10^{5} \chi_{0}$.

\begin{tabular}{lccc}
\hline \hline & & \multicolumn{3}{c}{ Models } \\
Ratio & W49A center & $P=10^{7} \mathrm{~cm}^{-3} \mathrm{~K}$ & $P=10^{8} \mathrm{~cm}^{-3} \mathrm{~K}$ \\
\hline $\mathrm{CN} / \mathrm{HCN}$ & 0.55 & 42.4 & 7.1 \\
$\mathrm{HCO} / \mathrm{H}^{13} \mathrm{CO}^{+}$ & $43.2-75.9$ & 31.5 & 5.0 \\
$\mathrm{CO}^{+} / \mathrm{H}^{13} \mathrm{CO}^{+}$ & $<0.56$ & 0.81 & 0.86 \\
$\mathrm{CS} / \mathrm{HCS}^{+}$ & $76-108$ & 7151 & 5696 \\
$\mathrm{SO}^{+} / \mathrm{SO}_{2}$ & $<0.02$ & 6564 & 4892 \\
\hline
\end{tabular}

ratio of $<0.02$ that we measured toward the center of W49A (based on the beam-averaged estimate for $\mathrm{SO}_{2}$ in Table 2) is at least an order of magnitude below the value measured in several PDRs, such as in the Orion Bar $\left(\left[\mathrm{SO}^{+}\right] /\left[\mathrm{SO}_{2}\right] \sim 0.4-1\right.$, Fuente et al. 2003). This difference may also be interpreted as the effect of shocks on the $\mathrm{SO}_{2}$ and $\mathrm{SO}^{+}$abundances toward the W49 center.

The importance of FUV irradiation in the chemistry of W49A can be probed by comparing the observed column density ratios to predictions of PDR models. Vastel et al. (2001) derive a radiation field toward the $\mathrm{W} 49 \mathrm{~A}$ center that is equivalent to $\chi=3.5 \times 10^{5} \chi_{0}$ in Draine (1978) units, with $\chi_{0}=$ $2.7 \times 10^{-3} \mathrm{erg} \mathrm{s}^{-1} \mathrm{~cm}^{-2}$. We use the 1.4 .4 version of the Meudon code (Le Petit et al. 2006; Goicoechea \& Le Bourlot 2007; Le Bourlot et al. 2012) to calculate column densities for the radiation field derived by Vastel et al. (2001) for expected densities of $10^{5} \mathrm{~cm}^{-3}$ and $10^{6} \mathrm{~cm}^{-3}$ and temperatures of order $100 \mathrm{~K} \mathrm{de}-$ rived by previous studies, corresponding to isobaric models with pressures of $10^{7} \mathrm{~cm}^{-3} \mathrm{~K}$ and $10^{8} \mathrm{~cm}^{-3} \mathrm{~K}$. We apply a cosmic-ray ionization rate of $2 \times 10^{-16} \mathrm{~s}^{-1} / \mathrm{H}_{2}$ molecule (Hollenbach et al. 2012). Predictions of column density ratios of selected species are summarized in Table 5.

The $[\mathrm{HCO}] /\left[\mathrm{H}^{13} \mathrm{CO}^{+}\right]$and $\left[\mathrm{CO}^{+}\right] /\left[\mathrm{H}^{13} \mathrm{CO}^{+}\right]$line ratios may be consistent with the $10^{7} \mathrm{~cm}^{-3} \mathrm{~K}$ pressure PDR model, showing that the abundance of some species may be described by PDR chemistry. The importance of PDR chemistry toward the region in W49A that we analyze here is further confirmed by Spitzer $8 \mu \mathrm{m}$ observations analyzed by Peng et al. (2010), showing emission along arcs and other structures toward most of the region where extended emission is seen in PDR tracers such as $\mathrm{C}_{2} \mathrm{H}$ and $\mathrm{CN}$. However, the $[\mathrm{CN}] /[\mathrm{HCN}],[\mathrm{CS}] /\left[\mathrm{HCS}^{+}\right]$, and $\left[\mathrm{SO}^{+}\right] /\left[\mathrm{SO}_{2}\right]$ column density ratios clearly show that the chemistry of the W49A center cannot be explained by a PDR component only, and different effects such as shocks also play an important role. The orders of magnitude difference seen in the $[\mathrm{CS}] /\left[\mathrm{HCS}^{+}\right]$and $\left[\mathrm{SO}^{+}\right] /\left[\mathrm{SO}_{2}\right]$ column density ratios may suggest the importance of shock chemistry. The result that the abundances (and abundance ratios) of sulphur-bearing molecules cannot be explained by a predominantly PDR chemistry is not surprising. Sulphur is released from grains into the gas phase in high temperature regions (such as PDRs and hot cores), but can also be evaporated by shocks. The regions with different chemistries (and different evaporation processes of sulphur from the grains) result in very different abundances for the sulphur-bearing species. This has been observationally confirmed toward regions where the different types of chemistries can more easily be disentangled. One example is Orion KL, where very different abundance ratios of sulphur-bearing molecules have been measured toward the plateau region (dominated by shock chemistry) and the extended ridge (dominated by PDR chemistry) as presented in Tercero et al. (2010).
Table 6. Comparison of the column density ratios measured toward the center of W49A to values measured toward regions with shock chemistry.

\begin{tabular}{lccccc}
\hline \hline Ratio & $\begin{array}{c}\text { W49A } \\
\text { center }\end{array}$ & $\begin{array}{c}\text { Orion KL } \\
\text { Plateau }\end{array}$ & L1157 & W3 IRS5 & $\begin{array}{c}\text { NGC 1333 } \\
\text { IRAS 4A }\end{array}$ \\
\hline $\mathrm{OCS} / \mathrm{CS}$ & $0.9-1.3$ & 5 & 0.2 & 0.4 & \\
$\mathrm{CS} / \mathrm{HCS}^{+}$ & $76-108$ & 50 & 63 & 321 & \\
$\mathrm{CS} / \mathrm{HCO}^{+}$ & $0.5-1.0$ & 5.6 & 3.7 & 1.1 & 19.5 \\
$\mathrm{CS} / \mathrm{H}_{2} \mathrm{CO}$ & $1.6-2.3$ & 0.1 & $0.3-0.9$ & 2.4 & 0.6 \\
$\mathrm{HCO}^{+} / \mathrm{HCS}^{+}$ & $90-165$ & 9 & 17 & 304 & \\
$\mathrm{H}_{2} \mathrm{CO} / \mathrm{H}_{2} \mathrm{CS}$ & $5.0-5.1$ & 18 & $2-5.3$ & 6.2 & \\
$\mathrm{SiO} / \mathrm{H}_{2} \mathrm{CO}$ & 0.1 & 0.8 & $0.1-0.3$ & 0.06 & 0.04 \\
$\mathrm{SO}_{2} / \mathrm{H}_{2} \mathrm{CO}$ & $35.4-36.7$ & 140 & $0.4-1$ & 64.5 & $<0.55$ \\
\hline
\end{tabular}

References. Orion KL plateau $\mathrm{SiO} / \mathrm{H}_{2} \mathrm{CO}$ and $\mathrm{SO}_{2} / \mathrm{H}_{2} \mathrm{CO}$ (Persson et al. 2007), Orion KL plateau other column density ratios (Tercero et al. 2010); L1157 position B1 (Bachiller \& Pérez Gutiérrez 1997); W3 IRS5 (Helmich \& van Dishoeck 1997); NGC 1333 IRAS 4A outflow (Blake et al. 1995).

Based on this comparison we conclude that UV irradiation contributes to the chemistry of the W49A center, but it is not the dominant effect.

\subsection{Comparison to regions with shock chemistry}

As we have shown above, the chemistry toward the W49A center is mostly related to effects other than FUV irradiation. Shocks related to outflows and winds of the young massive stars and protostars in the central stellar cluster are another possible effect that impact the chemistry in the W49 center. The importance of shocks in W49A has previously been studied by Peng et al. (2010) through their relation to expanding shells identified in ${ }^{13} \mathrm{CO}$ data, and by Nagy et al. (2012) as a possible contribution to the gas heating. Here we select a sample of regions with shock chemistry for a comparison to the observed column density ratios (Table 6).

The Orion KL is a well-studied, high-mass star-forming region at a distance of $\sim 414 \mathrm{pc}$ (Menten et al. 2007). Spectral line surveys toward Orion KL show a component (plateau) related to outflows and shocks (e.g., Tercero et al. 2010, and references therein). We use the $\mathrm{OCS} / \mathrm{CS}, \mathrm{CS} / \mathrm{HCS}^{+}, \mathrm{CS} / \mathrm{HCO}^{+}, \mathrm{CS} / \mathrm{H}_{2} \mathrm{CO}$, $\mathrm{HCO}^{+} / \mathrm{HCS}^{+}$, and $\mathrm{H}_{2} \mathrm{CO} / \mathrm{H}_{2} \mathrm{CS}$ column density ratios measured for the Orion KL plateau by Tercero et al. (2010). The $\mathrm{SiO} / \mathrm{H}_{2} \mathrm{CO}$ and $\mathrm{SO}_{2} / \mathrm{H}_{2} \mathrm{CO}$ ratios correspond to the low-velocity flow component reported by Persson et al. (2007).

The low-mass Class 0 protostar L1157 drives a strong molecular outflow (e.g., Bachiller \& Pérez Gutiérrez 1997, and references therein). Its blue lobe has a heating rate related to shocks that is comparable to that in the shocked region of Orion KL. The column density ratios shown in Table 6 correspond to the B 1 position of the blue lobe measured by Bachiller \& Pérez Gutiérrez (1997).

W3 IRS5 is a bright infrared source located in the W3 starforming region at a distance of $2.0 \mathrm{kpc}$ (e.g., Chavarría et al. 2010 and references therein). The embedded young massive stars drive several outflows, which affect the chemistry of the region. In Table 6 we show the column density ratios measured by Helmich \& van Dishoeck (1997).

The NGC 1333 star-forming region is located at a distance of 253 pc (Hirota et al. 2008) and contains several low-mass protostars, including IRAS 4A, which is also a source of outflows. 


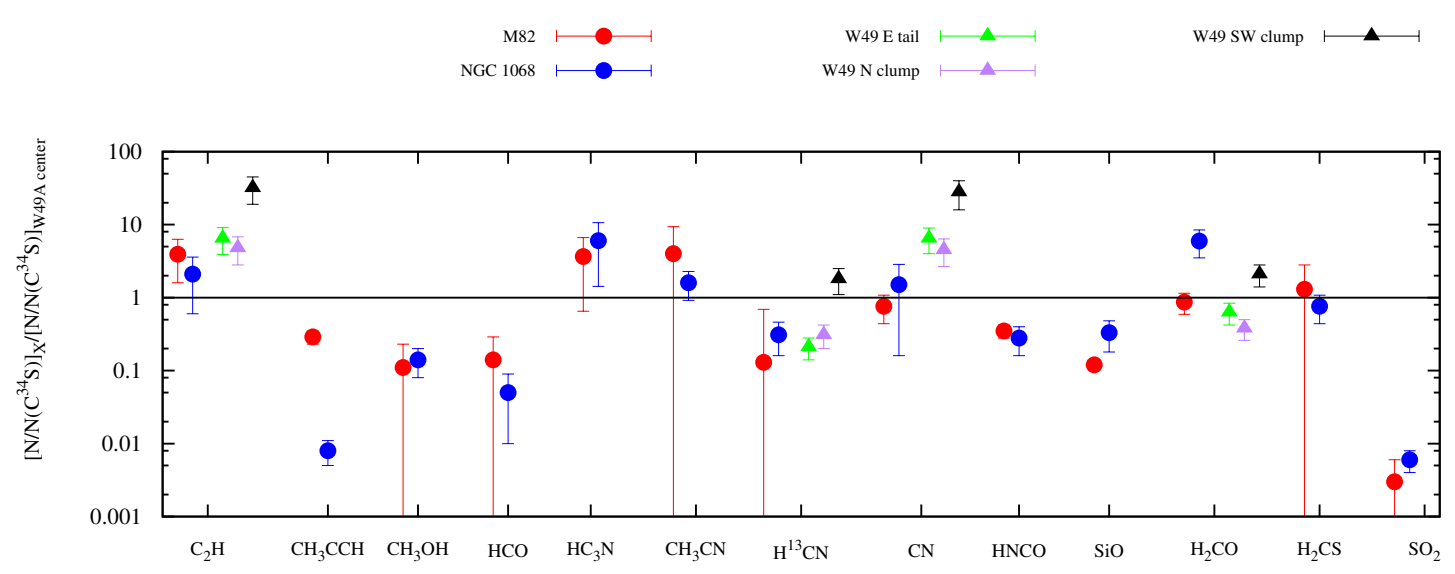

Fig. 15. Comparison of column density ratios (with respect to that of $\mathrm{C}^{34} \mathrm{~S}$ ) estimated in $\mathrm{W} 49 \mathrm{~A}$ and its subregions to those measured in the starburst galaxy M82 (Aladro et al. 2011b) and in the AGN NGC 1068 (Aladro et al. 2013). The error bars for HNCO and SiO toward M82 and those for $\mathrm{H}_{2} \mathrm{CO}, \mathrm{H}_{2} \mathrm{CS}$, and $\mathrm{SO}_{2}$ toward NGC 1068 are an underestimate of the error bars for the ratios, as these values were calculated based on upper limits for the column densities.

The column density ratios toward this source in Table 6 are from Blake et al. (1995).

The column density ratios observed toward the W49A center are closest to those measured toward the W3 IRS5 source, and match within a factor of 3 . The lower limit that we derive for the $\mathrm{CS} / \mathrm{HCS}^{+}$column density ratio is also close to those toward the Orion KL plateau and the $\mathrm{L} 1157$ regions. While the CS/HCS ${ }^{+}$ column density is not explained by any of the PDR models shown above, they match those observed toward regions with shock chemistry reasonably well. The $\mathrm{CS} / \mathrm{H}_{2} \mathrm{CO}$ column density ratios are also consistent with those measured toward L1157 (within a factor of 2) and NGC 1333 IRAS 4A (within a factor of 3). The $\mathrm{SiO} / \mathrm{H}_{2} \mathrm{CO}$ and $\mathrm{H}_{2} \mathrm{CO} / \mathrm{H}_{2} \mathrm{CS}$ column density ratios are very similar to that measured toward L1157. The largest differences between the regions compared in Table 6 are seen in the $\mathrm{SO}_{2} / \mathrm{H}_{2} \mathrm{CO}$ line ratios. As we have shown in the population diagram analysis, the $\mathrm{SO}_{2}$ lines probably originate in clumps smaller than the beam-size (Table 2). Therefore, the large differences in the $\mathrm{SO}_{2} / \mathrm{H}_{2} \mathrm{CO}$ column density ratio between the regions may be related to the combination of the different spatial scales that correspond to the observations and to the clumpy structure of $\mathrm{SO}_{2}$.

Even this simple comparison of observed column density ratios shows the importance of shocks in the chemistry of the center of W49A. Further evidence could be given by a comparison to shock models and by smaller scale observations of sulphurbearing molecules in particular.

\subsection{Comparison to starburst galaxies and AGNs}

Several molecules seen in our line survey have been detected in starburst galaxies and AGNs. A comparison between these regions and W49A is shown in Fig. 15, based on line surveys toward the starburst galaxy M82 (Aladro et al. 2011b, carried out using the IRAM-30 m with beam sizes of $14^{\prime \prime}-19^{\prime \prime}$ at $\sim 2 \mathrm{~mm}$ and $9^{\prime \prime}-10^{\prime \prime}$ at $\sim 1.3 \mathrm{~mm}$ ) and the AGN NGC 1068 (Aladro et al. 2013, carried out using the IRAM-30 m with a $\sim 21^{\prime \prime}-29^{\prime \prime}$ beam). For the column density ratios toward the center, the beam-averaged column densities are used in the case of species with multiple detected transitions, and not the value corresponding to the best-fit source size indicated by the population diagrams (see Sect. 3.4). We use $\mathrm{C}^{34} \mathrm{~S}$ as a reference for the column density ratios to be consistent with Aladro et al. (2011b) and
Aladro et al. (2013). $\mathrm{C}^{34} \mathrm{~S}$ is also a dense gas tracer and is expected to be optically thin. The error bars of the ratios presented in Fig. 15 are based on the error bars for $N(\mathrm{X}) / N\left(\mathrm{C}^{34} \mathrm{~S}\right)$ for M82 (Aladro et al. 2011b), the column densities for NGC 1068 (Aladro et al. 2013), and the column densities presented here and in Nagy et al. (2012). For some of the abundance ratios this is only a lower limit of the error, as those were calculated using column density upper limits given for M82 ( $\mathrm{HNCO}$ and $\mathrm{SiO}$, Aladro et al. 2011b) and for NGC $1068\left(\mathrm{H}_{2} \mathrm{CO}, \mathrm{H}_{2} \mathrm{CS}\right.$, and $\mathrm{SO}_{2}$, Aladro et al. 2013). Although a comparison of Galactic scales to those probed in external galaxies, such as a scale of $\sim 0.8 \mathrm{pc}$ for W49A to the 1.5-2 kpc for NGC 1068 (Aladro et al. 2013) and 158-333 pc for M82 (Aladro et al. 2011b) is an oversimplification, a few conclusions can be drawn.

The most similar abundances with respect to that of $\mathrm{C}^{34} \mathrm{~S}$ between $\mathrm{W} 49 \mathrm{~A}$ and the $\mathrm{AGN}$ and starburst environments are seen for $\mathrm{C}_{2} \mathrm{H}, \mathrm{HC}_{3} \mathrm{~N}, \mathrm{CH}_{3} \mathrm{CN}, \mathrm{CN}, \mathrm{H}_{2} \mathrm{CS}$, and for $\mathrm{H}_{2} \mathrm{CO}$ in the case of $\mathrm{M} 82$. In the case of $\mathrm{H}_{2} \mathrm{CO}$, the northern clump, eastern tail, and southwest clump regions also show similar abundances, w.r.t. $\mathrm{C}^{34} \mathrm{~S}$ as M82 and the W49A center. $\mathrm{C}_{2} \mathrm{H}, \mathrm{CN}$, and $\mathrm{H}_{2} \mathrm{CO}$ are detected with a large spatial extent in W49A. The similarity of the fractional abundance of these species between M82 and W49A, and for $\mathrm{C}_{2} \mathrm{H}$ and $\mathrm{CN}$, as well as to that toward NGC 1068, suggests that they are good tracers to compare physical and chemical properties of in Galactic star-forming regions at scales of $<1 \mathrm{pc}$ and global star formation seen in external galaxies on scales of $>1 \mathrm{kpc}$.

Among the four subregions of W49A, the largest differences compared to the other regions in the observed fractional abundances w.r.t. $C^{34} S$ are seen for the southwest clump. As $C^{34} S$ is a dense-gas tracer, the explanation is possibly related to the lower average $\mathrm{H}_{2}$ volume density of the southwest clump compared to the other regions, which is traced by $\mathrm{HCN} 3-2$ and 4-3 line intensity ratios (Nagy et al. 2012).

The largest difference compared both to the AGN and starburst examples is seen in the $\mathrm{SO}_{2}$ fractional abundance. This is probably related to the difference between the spatial scales of the observations. $\mathrm{SO}_{2}$ and most sulphur-bearing species in W49A are detected in a $\sim 20^{\prime \prime} \times 20^{\prime \prime}$ region around the center and therefore are least likely to trace "global" properties of $>\mathrm{kpc}$ regions, such as the more spatially extended species $\mathrm{C}_{2} \mathrm{H}, \mathrm{CN}$, and $\mathrm{H}_{2} \mathrm{CO}$. Other species with a similar spatial extent to $\mathrm{SO}_{2}$ also show significant differences in Fig. 15; i.e., $\mathrm{CH}_{3} \mathrm{CCH}, \mathrm{HCO}$, and $\mathrm{SiO}$. 
Z. Nagy et al.: Physical and chemical differentiation of W49A

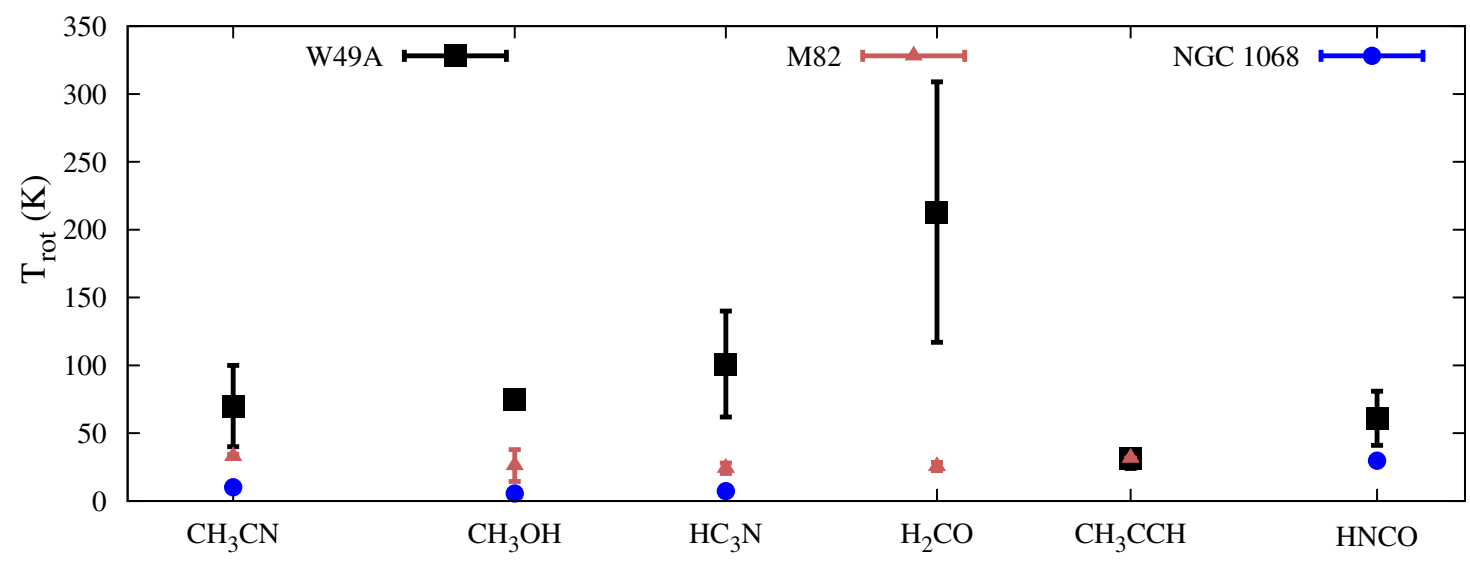

Fig. 16. Comparison of the rotational temperatures measured toward the W49A center and those measured toward M82 (Aladro et al. $2011 \mathrm{~b}$ ) and toward NGC 1068 (Aladro et al. 2013).

Apart from the abundances, the excitation conditions can also be used to compare W49A to external galaxies. We presented an excitation analysis for 14 molecules, and estimated rotational and excitation temperatures. These temperatures can also be compared to those found toward M82 and NGC 1068. In the following, we use the derived rotational temperatures to be consistent with the values derived toward these two galaxies, and to use the values that correspond to uniform beam filling equivalent to emitting regions of $\sim 0.8 \mathrm{pc}$ (the JCMT beam at the observed frequencies). Figure 16 shows the comparison of the species that have a measured excitation temperature toward the W49A center, M82 (Aladro et al. 2011b), and NGC 1068 (Aladro et al. 2013). Most of the rotational temperatures measured toward the W49A center are significantly higher (factors of 2-10) compared to those measured toward M82 and NGC 1068. This is not surprising because of the very different spatial scales that the measurements correspond to. $\mathrm{CH}_{3} \mathrm{CCH}$ is an exception as the rotational temperature of this molecule is very close to the value derived toward M82. Apart from $\mathrm{CH}_{3} \mathrm{CCH}$ toward $\mathrm{M} 82, \mathrm{CH}_{3} \mathrm{CN}$ toward $\mathrm{M} 82$ and $\mathrm{HNCO}$ toward NGC 1068 have rotational temperatures close to the value measured toward the W49A center. The largest difference is seen for $\mathrm{H}_{2} \mathrm{CO}$, which is not the case when comparing the abundances toward W49A, M82, and NGC 1068. This comparison toward $\mathrm{W} 49 \mathrm{~A}$ is biased toward the highest density region with the highest excitation. For a better comparison, more transitions toward the off-center regions (such as the northern clump, eastern tail, and southwest clump) could be compared to similar observations in starburst galaxies and AGNs.

\section{Summary}

We presented results from the SLS survey carried out using the JCMT at an angular resolution of $\sim 15^{\prime \prime}$ in the $330-373 \mathrm{GHz}$ frequency range toward the luminous and massive star-forming region $\mathrm{W} 49 \mathrm{~A}$. Our maps cover a $2 \times 2$ arcmin region around the center of W49A, including the regions referred to as the center, the eastern tail, the northern clump, and the southwest clump, which were selected based on a combination of the morphology and the kinematics of the detected molecular lines; these regions were also previously used in Roberts et al. (2011). Our results and conclusions can be summarized as follows:

- The detected 255 transitions correspond to 63 species including shock-, and PDR-tracers, and complex organic molecules. Excitation conditions can be probed using multiple detected transitions for 14 species.

- The species detected with the largest spatial extent include $\mathrm{CO}$ (and its ${ }^{13} \mathrm{CO}$ and $\mathrm{C}^{17} \mathrm{O}$ isotopologues), $\mathrm{N}_{2} \mathrm{H}^{+}$, and $\mathrm{H}_{2} \mathrm{CO}$; the dense gas tracers including $\mathrm{CS}, \mathrm{HCN}, \mathrm{HNC}$, and $\mathrm{HCO}^{+}$; PDR tracers such as $\mathrm{CN}$ and $\mathrm{C}_{2} \mathrm{H}$.

- The most spatially extended species reveal a complex spatial and kinematic structure, covering the velocity range of $[-5$, $+20] \mathrm{km} \mathrm{s}^{-1}$. Most of the observed lines toward the center are double-peaked or asymmetric, with signatures of infall and outflow motions. Mostly blue-shifted emission is detected toward the eastern tail region, mostly red-shifted emission toward the northern clump region, while emission toward the southwest clump is detected around the source velocity.

- Based on column density ratios of characteristic species, a combination of shock and PDR chemistry affects the physical and chemical structure of the central $17^{\prime \prime} \times 17^{\prime \prime}$ region, while UV-irradiation dominates outside of this region, including the northern clump, eastern tail, and southwest clump regions. A preliminary comparison to a starburst galaxy and to an AGN suggests similar $\mathrm{C}_{2} \mathrm{H}, \mathrm{CN}$, and $\mathrm{H}_{2} \mathrm{CO}$ abundances between the $\sim 0.8 \mathrm{pc}$ scale probed for $\mathrm{W} 49 \mathrm{~A}$ and the $>1 \mathrm{kpc}$ regions in external galaxies with global star formation, suggesting these to be best tracers for a comparison of physical and chemical properties of star formation seen on different scales.

In the future, observations with interferometers, such as with ALMA, will probe the chemistry of the W49A region on the smaller scales, indicating the differences between the individual components of the source that are not resolved by single dish telescopes.

Acknowledgements. We thank the referee for the useful suggestions and the editor Malcolm Walmsley for additional comments. We thank Kuo-Song Wang for providing his population diagram code and John Black for useful comments on an earlier version of this manuscript.

\section{References}

Aalto, S., Costagliola, F., van der Tak, F., \& Meijerink, R. 2011, A\&A, 527, A69 Aladro, R., Martín-Pintado, J., Martín, S., Mauersberger, R., \& Bayet, E. 2011a, A\&A, 525, A89

Aladro, R., Martín, S., Martín-Pintado, J., et al. 2011b, A\&A, 535, A84

Aladro, R., Viti, S., Bayet, E., et al. 2013, A\&A, 549, A39

Alves, J., \& Homeier, N. 2003, ApJ, 589, 45

Bachiller, R., \& Pérez Gutiérrez, M. 1997, ApJ, 487, L93

Blake, G. A., Sandell, G., van Dishoeck, E. F., et al. 1995, ApJ, 441, 689

Buckle, J. V., Hills, R. E., Smith, H., et al. 2009, MNRAS, 399, 1026 
Chavarría, L., Herpin, F., Jacq, T., et al. 2010, A\&A, 521, L37

Churchwell, E., \& Hollis, J. M. 1983, ApJ, 272, 591

Conti, P. S., \& Blum, R. D. 2002, ApJ, 564, 827

Costagliola, F., \& Aalto, S. 2010, A\&A, 515, A71

De Pree, C. G., Mehringer, D. M., \& Goss, W. M. 1997, ApJ, 482, 307

De Pree, C. G., Wilner, D. J., Mercer, A. J., et al. 2004, ApJ, 600, 286

De Pree, C. G., Wilner, D. J., Deblasio, J., Mercer, A. J., \& Davis, L. E. 2005, ApJ, 624, 101

Draine, B. T. 1978, ApJS, 36, 595

Frerking, M. A., Wilson, R. W., Linke, R. A., \& Wannier, P. G. 1980, ApJ, 240, 65

Fuente, A., Martín-Pintado, J., Cernicharo, J., \& Bachiller, R. 1993, A\&A, 276, 473

Fuente, A., Rodríguez-Franco, A., \& Martín-Pintado, J. 1996, A\&A, 312, 599

Fuente, A., Rodríguez-Franco, A., García-Burillo, S., Martín-Pintado, J., \& Black, J. H. 2003, A\&A, 406, 899

Galvan-Madrid, R., Liu, H. B., Zhang, Z.-Y., et al. 2013, ApJ, 779, 121

Gerin, M., Goicoechea, J. R., Pety, J., \& Hily-Blant, P. 2009, A\&A, 494, 977

Gerin, M., de Luca, M., Black, J., et al. 2010, A\&A, 518, L110

Ginard, D., González-García, M., Fuente, A., et al. 2012, A\&A, 543, A27

Goicoechea, J. R., \& Le Bourlot, J. 2007, A\&A, 467, 1

Goicoechea, J. R., Pety, J., \& Gerin, M. 2006, A\&A, 456, 565

Goldsmith, P. F., \& Langer, W. D. 1999, ApJ, 517, 209

Gordon, M. A., \& Walmsley, C. M. 1990, ApJ, 365, 606

Gusdorf, A., Cabrit, S., Flower, D. R., \& Pineau Des Forêts, G. 2008, A\&A, 482, 809

Gwinn, C. R., Moran, J. M., \& Reid, M. J. 1992, ApJ, 393, 149

Helmich, F. P., \& van Dishoeck, E. F., 1997, A\&AS, 124, 205

Herbst, E., \& Van Dishoeck, E. F. 2009, ARA\&A, 47, 427

Hirota, T., Bushimata, T., Choi, Y. K., et al. 2008, PASJ, 60, 37

Hollenbach, D., Kaufman, M. J., Neufeld, D., Wolfire, M., \& Goicoechea, J. R. 2012, ApJ, 754, 105

Ikeda, M., Ohishi, M., Nummelin, A., et al. 2001, ApJ, 560, 792

Le Bourlot, J., Le Petit, F., Pinto, C., Roueff, E., \& Roy, F. 2012, A\&A, 541, A76

Le Petit, F., Nehmé, C., Le Bourlot, J., \& Roueff, E. 2006, ApJS, 164, 506

Lindberg, J. E., Aalto, S., Costagliola, F., et al. 2011, A\&A, 527, A150

Lucas, R., \& Liszt, H. S. 2000, A\&A, 355, 327

Mangum, J. G., \& Wootten, A. 1993, ApJS, 89, 123

Mangum, J. G., Darling, J., Menten, K. M., \& Henkel, C. 2008, ApJ, 673, 832

Menten, K. M., Reid, M. J., Forbrich, J., \& Brunthaler, A. 2007, A\&A, 474, 515

Müller, H. S. P., Schlöder, F., Stutzki, J., \& Winnewisser, G. 2005, J. Mol. Struct., 742,215

Nagy, Z., Van der Tak, F. F. S., Fuller, G. A., Spaans, M., \& Plume, R. 2012, A\&A, 542, A6
Neufeld, D. A., Goicoechea, J. R., Sonnentrucker, P., et al. 2010, A\&A, 521, L10

Nisini, B., Codella, C., Giannini, T., et al. 2007, A\&A, 462, 163

Peng, T., Wyrowski, F., van der Tak, F. F. S., Menten, K. M., \& Walmsley, C. M. 2010, A\&A, 520, A84

Persson, C. M., Olofsson, A. O. H., Koning, N., et al. 2007, A\&A, 476, 807

Pickett, H. M., Poynter, R. L., Cohen, E. A., et al. 1998, J. Quant. Spectr. Radiat. Transf., 60, 883

Plume, R., Fuller, G. A., Helmich, F., et al. 2007, PASP, 119, 102

Rizzo, J. R., Fuente, A., Rodríguez-Franco, A., \& García-Burillo, S. 2003, ApJ, 597, 153

Rizzo, J. R., Fuente, A., \& García-Burillo, S. 2005, ApJ, 634, 1133

Roberts, H., \& Millar, T. J. 2007, A\&A, 471, 849

Roberts, H., Van der Tak, F. F. S., Fuller, G. A., Plume, R., \& Bayet, E. 2011, A\&A, 525, A107

Rodríguez-Fernández, N. J., Tafalla, M., Gueth, F., \& Bachiller, R. 2010, A\&A, 516, A98

Schilke, P., Walmsley, C. M., Pineau des Forets, G., \& Flower, D. R. 1997, A\&A 321, 293

Schilke, P., Pineau des Forets, G., Walmsley, C. M., \& Martín-Pintado, J. 2001, A\&A, 372, 291

Serabyn, E., Guesten, R., \& Schulz, A. 1993, ApJ, 413, 571

Sievers, A. W., Mezger, P. G., Bordeon, M. A., et al. 1991, A\&A, 251, 231

Simon, R., Jackson, J. M., Clemens, D. P., Bania, T. M., \& Heyer, M. H. 2001, ApJ, 551, 747

Solomon, P. M., \& Vanden Bout, P. A. 2005, ARA\&A, 43, 677

Solomon, P. M., Downes, D., Radford, S. J. E., \& Barrett, J. W. 1997, ApJ, 478, 144

Tercero, B., Cernicharo, J., Pardo, J. R., \& Goicoechea, J. R. 2010, A\&A, 517, A96

Turner, B. E. 1992, ApJ, 396, L107

Van der Tak, F. F. S., Aalto, S., \& Meijerink, R. 2008, A\&A, 477, L5

Van der Werf, P. P., Isaak, K. G., Meijerink, R., et al. 2010, A\&A, 518, L42

Van der Wiel, M. H. D. 2011, Ph.D. Thesis, Univ. Groningen

Vastel, C., Spaans, M., Ceccarelli, C., Tielens, A. G. G. M., \& Caux, E. 2001, A\&A, 376, 1064

Wakelam, V., Castets, A., Ceccarelli, C., et al. 2004, A\&A, 413, 609

Ward-Thompson, D., \& Robson, E. I. 1990, MNRAS, 244, 458

Welch, W. J., Dreher, J. W., Jackson, J. M., Terebey, S., \& Vogel, S. N. 1987, Science, 238,1550

Wilner, D. J., De Pree, C. G., Welch, W. J., \& Goss, W. M. 2001, ApJ, 550, L81

Wu, S.-W., Bik, A., Henning, T., et al. 2014, A\&A, 568, L13

Wyrowski, F., Schilke, P., \& Walmsley, C. M. 1999, A\&A, 341, 882

Zhang, B., Reid, M. J., Menten, K. M., et al. 2013, ApJ, 775, 79

Ziurys, L. M., Snell, R. L., \& Dickman, R. L. 1989, ApJ, 341, 857 


\section{Appendix A: The detected lines toward the center of W49A}

Table A.1. The detected species toward the central position of the SLS field.

\begin{tabular}{|c|c|c|c|c|c|c|c|c|}
\hline Molecule & Transition & $\begin{array}{l}\text { Freq. } \\
\text { (MHz) }\end{array}$ & $\begin{array}{l}E_{\text {up }} \\
(\mathrm{K})\end{array}$ & $\begin{array}{l}A_{\mathrm{ij}} \\
\left(\mathrm{s}^{-1}\right)\end{array}$ & $\begin{array}{l}\int T_{\mathrm{mb}} \mathrm{d} v \\
\left(\mathrm{~K} \mathrm{~km} \mathrm{~s}^{-1}\right)\end{array}$ & $\begin{array}{l}V_{\mathrm{LSR}} \\
\left(\mathrm{km} \mathrm{s}^{-1}\right)\end{array}$ & $\begin{array}{l}F W H M \\
\left(\mathrm{~km} \mathrm{~s}^{-1}\right)\end{array}$ & $\begin{array}{l}T_{\text {peak }} \\
(\mathrm{K})\end{array}$ \\
\hline HNCO & $15_{0,15}-14_{0,14}$ & 329664.4 & 126.6 & $5.04 \times 10^{-4}$ & $12.09 \pm 0.95$ & $9.68 \pm 0.64$ & $16.57 \pm 1.50$ & $0.69 \pm 0.14$ \\
\hline${ }^{34} \mathbf{S O}_{2}$ & $8_{2,6}-7_{1,7}$ & 330191.1 & 42.8 & $1.19 \times 10^{-4}$ & $8.90 \pm 0.58$ & $10.70 \pm 0.42$ & $13.31 \pm 1.02$ & $0.63 \pm 0.12$ \\
\hline${ }^{13} \mathrm{CO}^{\star}$ & $3-2$ & 330588.0 & 31.7 & $2.19 \times 10^{-6}$ & $616.28 \pm 1.01$ & $5.31 \pm 0.01$ & $15.88 \pm 0.03$ & $36.46 \pm 1.80$ \\
\hline${ }^{34} \mathrm{SO}_{2}$ & $21_{2,20}-21_{1,21}$ & 330667.6 & 218.9 & $1.45 \times 10^{-4}$ & $5.39 \pm 0.61$ & $10.70 \pm 0.87$ & $14.77 \pm 1.66$ & $0.34 \pm 0.07$ \\
\hline $\mathrm{CH}_{3} \mathrm{OH}^{\star}$ & $8_{-3}-9_{-2} E$ & 330793.9 & 146.3 & $5.39 \times 10^{-5}$ & $4.62 \pm 0.45$ & $9.85 \pm 0.86$ & $17.44 \pm 1.80$ & $0.25 \pm 0.09$ \\
\hline HNCO & $15_{1,14}-14_{1,13}$ & 330848.6 & 170.3 & $5.01 \times 10^{-4}$ & $7.77 \pm 0.51$ & $11.35 \pm 0.49$ & $14.65 \pm 1.08$ & $0.50 \pm 0.10$ \\
\hline $\mathrm{CH}_{3} \mathrm{CN}^{\star}$ & $18_{3}-17_{3}$ & 331014.3 & 215.2 & $3.07 \times 10^{-3}$ & $2.59 \pm 0.25$ & $9.68 \pm 1.00$ & $10.51 \pm 1.00$ & $0.23 \pm 0.11$ \\
\hline $\mathrm{CH}_{3} \mathrm{CN}^{\star}$ & $18_{2}-17_{2}$ & 331046.1 & 179.5 & $3.12 \times 10^{-3}$ & $3.53 \pm 0.77$ & $11.88 \pm 1.32$ & $13.05 \pm 2.96$ & $0.25 \pm 0.10$ \\
\hline $\mathrm{CH}_{3} \mathrm{CN}^{\star}$ & $18_{0}-17_{0}$ & 331071.5 & 151.0 & $3.16 \times 10^{-3}$ & $9.50 \pm 0.26$ & $11.91 \pm 1.00$ & $17.90 \pm 1.00$ & $0.50 \pm 0.11$ \\
\hline $\mathrm{CH}_{3} \mathrm{OH}^{\star}$ & $11_{1}-11_{0} E^{\mp}$ & 331502.4 & 169.0 & $3.93 \times 10^{-4}$ & $15.60 \pm 0.64$ & $8.11 \pm 0.31$ & $15.01 \pm 0.72$ & $0.98 \pm 0.12$ \\
\hline $\mathbf{S O}_{2}$ & $11_{6,6}-12_{5,7}$ & 331580.2 & 149.0 & $4.35 \times 10^{-5}$ & $17.80 \pm 0.61$ & $10.77 \pm 0.24$ & $14.73 \pm 0.61$ & $1.14 \pm 0.12$ \\
\hline $\mathrm{SO}_{2}^{\star}$ & $21_{2,20}-21_{1,21}$ & 332091.4 & 219.5 & $1.51 \times 10^{-4}$ & $52.14 \pm 0.69$ & $10.08 \pm 0.10$ & $16.28 \pm 0.26$ & $3.01 \pm 0.17$ \\
\hline${ }^{34} \mathbf{S O}_{2}$ & $23_{3,21}-23_{2,22}$ & 332173.6 & 275.1 & $2.54 \times 10^{-4}$ & $9.10 \pm 0.63$ & $11.94 \pm 0.47$ & $14.16 \pm 1.21$ & $0.60 \pm 0.13$ \\
\hline $\mathrm{SO}_{2}^{\star}$ & $4_{3,1}-3_{2,2}$ & 332505.2 & 31.3 & $3.29 \times 10^{-4}$ & $75.04 \pm 0.77$ & $9.11 \pm 0.08$ & $15.99 \pm 0.20$ & $4.41 \pm 0.15$ \\
\hline${ }^{34} \mathbf{S O}_{2}$ & $16_{4,12}-16_{3,13}$ & 332836.2 & 163.1 & $3.02 \times 10^{-4}$ & $17.88 \pm 1.02$ & $12.26 \pm 0.44$ & $16.58 \pm 1.23$ & $1.01 \pm 0.13$ \\
\hline $\mathbf{S}^{17} \mathbf{O}$ & $8_{9}-7_{8}$ & 333121.6 & 75.9 & $4.50 \times 10^{-4}$ & $3.89 \pm 0.67$ & $12.38 \pm 1.49$ & $15.91 \pm 2.71$ & $0.23 \pm 0.12$ \\
\hline${ }^{34} \mathbf{S O}^{\star}$ & $7_{8}-6_{7}$ & 333901.0 & 79.9 & $4.69 \times 10^{-4}$ & $47.01 \pm 0.42$ & $9.57 \pm 0.06$ & $14.00 \pm 0.15$ & $3.15 \pm 0.11$ \\
\hline $\mathbf{S O}_{2}^{\star}$ & $8_{2,6}-7_{1,7}$ & 334673.4 & 43.1 & $1.27 \times 10^{-4}$ & $42.19 \pm 0.69$ & $10.38 \pm 0.12$ & $15.60 \pm 0.30$ & $2.54 \pm 0.09$ \\
\hline $\mathrm{CH}_{3} \mathrm{OH}^{\star}$ & $2{ }_{2}-3_{1} \mathrm{~A}^{-}$ & 335133.7 & 44.7 & $2.69 \times 10^{-5}$ & $6.95 \pm 0.46$ & $12.41 \pm 0.66$ & $19.32 \pm 1.30$ & $0.34 \pm 0.09$ \\
\hline H & $33 \beta$ & 335207.3 & & & $5.19 \pm 0.65$ & $6.62 \pm 1.91$ & $30.50 \pm 4.37$ & $0.16 \pm 0.07$ \\
\hline $\mathrm{CH}_{3} \mathrm{CHO}$ & $18_{0,18,2}-17_{0,17,2}$ & 335318.1 & 154.9 & $1.30 \times 10^{-3}$ & $6.53 \pm 0.41$ & $11.02 \pm 0.61$ & $20.02 \pm 1.44$ & $0.31 \pm 0.06$ \\
\hline $\mathrm{CH}_{3} \mathrm{OH}^{\star}$ & $7_{1}-6_{1} A^{+}$ & 335582.0 & 79.0 & $1.63 \times 10^{-4}$ & $20.67 \pm 0.32$ & $7.68 \pm 0.11$ & $14.28 \pm 0.24$ & $1.36 \pm 0.11$ \\
\hline $\mathbf{S O}_{2}^{\star}$ & $23_{3,21}-23_{2,22}$ & 336089.2 & 276.0 & $2.67 \times 10^{-4}$ & $37.35 \pm 0.41$ & $10.02 \pm 0.08$ & $14.77 \pm 0.20$ & $2.38 \pm 0.10$ \\
\hline $\mathrm{HC}_{3} \mathbf{N}$ & $37-36$ & 336520.1 & 306.9 & $3.05 \times 10^{-3}$ & $7.16 \pm 0.62$ & $10.74 \pm 0.60$ & $14.57 \pm 1.64$ & $0.46 \pm 0.09$ \\
\hline SO & $11_{10}-10_{10}$ & 336553.8 & 142.9 & $6.12 \times 10^{-6}$ & $22.03 \pm 1.01$ & $9.40 \pm 0.31$ & $13.57 \pm 0.69$ & $1.52 \pm 0.08$ \\
\hline $\mathbf{S O}_{2}$ & $16_{7,9}-17_{6,12}$ & 336669.6 & 245.1 & $5.84 \times 10^{-5}$ & $16.22 \pm 0.11$ & $11.05 \pm 0.17$ & $11.46 \pm 0.34$ & $1.33 \pm 0.14$ \\
\hline $\mathrm{CH}_{3} \mathrm{OH}^{\star}$ & $12_{1}-12_{0} \mathrm{~A}^{\mp}$ & 336865.1 & 197.1 & $4.07 \times 10^{-4}$ & $14.71 \pm 0.44$ & $7.69 \pm 0.22$ & $15.21 \pm 0.53$ & $0.91 \pm 0.10$ \\
\hline $\mathbf{C}^{17} \mathbf{O}^{\star}$ & $3-2$ & 337062.0 & 32.4 & $4.30 \times 10^{-7}$ & $84.53 \pm 0.39$ & $7.39 \pm 0.03$ & $13.62 \pm 0.07$ & $5.83 \pm 0.32$ \\
\hline${ }^{33} \mathbf{S O}$ & $7_{8}-6_{7}$ & 337199.4 & 80.5 & $4.65 \times 10^{-4}$ & $19.13 \pm 0.64$ & $10.11 \pm 0.24$ & $14.68 \pm 0.59$ & $1.22 \pm 0.10$ \\
\hline $\mathbf{H C}_{3} \mathbf{N}, v_{7}=1$ & $J=37-36$ & 337344.7 & 628.5 & $3.05 \times 10^{-3}$ & $3.19 \pm 0.55$ & $14.02 \pm 1.04$ & $11.72 \pm 2.43$ & $0.26 \pm 0.09$ \\
\hline $\mathbf{C}^{34} \mathbf{S}^{\star}$ & $7-6$ & 337396.5 & 50.2 & $8.00 \times 10^{-4}$ & $24.98 \pm 0.56$ & $8.02 \pm 0.15$ & $13.79 \pm 0.35$ & $1.70 \pm 0.15$ \\
\hline${ }^{34} \mathbf{S O}^{\star}$ & $8_{8}-7_{7}$ & 337580.1 & 86.1 & $4.89 \times 10^{-4}$ & $46.74 \pm 0.43$ & $9.08 \pm 0.06$ & $13.66 \pm 0.14$ & $3.21 \pm 0.10$ \\
\hline $\mathbf{C H}_{3} \mathbf{O H}, v_{t}=1$ & $7_{7,1}-6_{6,1} \mathrm{~A}^{+}$ & 337643.9 & 365.4 & $1.69 \times 10^{-4}$ & $3.54 \pm 0.50$ & $9.49 \pm 1.20$ & $15.61 \pm 2.04$ & $0.21 \pm 0.06$ \\
\hline $\mathrm{CH}_{3} \mathrm{OCH}_{3}$ & $7_{4,3,0}-6_{3,4,0}$ & 337787.2 & 48.0 & $1.94 \times 10^{-4}$ & $1.80 \pm 0.53$ & $11.72 \pm 1.38$ & $10.13 \pm 3.81$ & $0.17 \pm 0.04$ \\
\hline${ }^{34} \mathrm{SO}$ & $3_{3}-2_{3}$ & 337892.2 & 25.3 & $1.40 \times 10^{-5}$ & $4.59 \pm 0.54$ & $10.81 \pm 0.70$ & $12.32 \pm 1.81$ & $0.35 \pm 0.08$ \\
\hline $\mathrm{H}_{2} \mathbf{C S}$ & $10_{1,10}-9_{1,9}$ & 338083.2 & 102.4 & $5.77 \times 10^{-4}$ & $7.31 \pm 0.57$ & $7.36 \pm 0.54$ & $13.65 \pm 1.17$ & $0.50 \pm 0.07$ \\
\hline $\mathrm{CH}_{3} \mathrm{OH}^{\star}$ & $7_{0}-6_{0} E$ & 338124.5 & 78.1 & $1.70 \times 10^{-4}$ & $15.87 \pm 0.88$ & $6.64 \pm 0.37$ & $13.31 \pm 0.85$ & $1.12 \pm 0.10$ \\
\hline $\mathrm{SO}_{2}^{\star}$ & $18_{4,14}-18_{3,15}$ & 338306.0 & 196.8 & $3.27 \times 10^{-4}$ & $29.82 \pm 0.39$ & $7.47 \pm 0.11$ & $18.52 \pm 0.28$ & $1.51 \pm 0.10$ \\
\hline $\mathrm{CH}_{3} \mathrm{OH}^{\star}$ & $7_{-1}-6_{-1} E$ & 338344.6 & 70.6 & $1.67 \times 10^{-4}$ & $23.99 \pm 0.31$ & $6.59 \pm 0.09$ & $13.53 \pm 0.21$ & $1.67 \pm 0.10$ \\
\hline $\mathrm{CH}_{3} \mathrm{OH}^{\star}$ & $7_{0}-6_{0} \mathrm{~A}^{+}$ & 338408.7 & 65.0 & $1.70 \times 10^{-4}$ & $22.13 \pm 0.30$ & $6.62 \pm 0.08$ & $12.26 \pm 0.19$ & $1.70 \pm 0.10$ \\
\hline $\mathrm{CH}_{3} \mathrm{OH}^{\star}$ & $7_{4,4}-6_{4,3} \mathrm{~A}^{-}$ & 338512.6 & 145.3 & $1.15 \times 10^{-4}$ & $10.32 \pm 0.48$ & $7.35 \pm 0.32$ & $13.83 \pm 0.72$ & $0.70 \pm 0.09$ \\
\hline $\mathrm{CH}_{3} \mathrm{OH}$ & $7_{3,4}-6_{3,3} \mathrm{~A}^{-}$ & 338543.2 & 114.8 & $1.39 \times 10^{-4}$ & $10.68 \pm 0.50$ & $7.97 \pm 0.33$ & $13.87 \pm 0.75$ & $0.72 \pm 0.09$ \\
\hline $\mathrm{SO}_{2}^{\star}$ & $20_{1,19}-19_{2,18}$ & 338611.8 & 198.9 & $2.87 \times 10^{-4}$ & $40.93 \pm 0.50$ & $6.95 \pm 0.09$ & $15.73 \pm 0.22$ & $2.44 \pm 0.10$ \\
\hline $\mathrm{CH}_{3} \mathrm{OH}$ & $7_{2}-6_{2} A^{+}$ & 338639.9 & 102.7 & $1.57 \times 10^{-4}$ & $7.93 \pm 0.90$ & $6.75 \pm 1.00$ & $11.79 \pm 1.00$ & $0.63 \pm 0.10$ \\
\hline $\mathrm{CH}_{3} \mathrm{OH}^{\star}$ & $7_{2}-6_{2} E$ & 338721.6 & 87.3 & $1.55 \times 10^{-4}$ & $21.52 \pm 0.27$ & $5.92 \pm 0.09$ & $13.16 \pm 0.20$ & $1.54 \pm 0.17$ \\
\hline${ }^{34} \mathbf{S O}_{2}$ & $14_{4,10}-14_{3,11}$ & 338785.7 & 134.5 & $3.08 \times 10^{-4}$ & $5.35 \pm 0.32$ & $11.23 \pm 0.38$ & $12.57 \pm 0.83$ & $0.40 \pm 0.04$ \\
\hline $\mathrm{SO}^{\star}$ & $3_{3}-3_{2}$ & 339341.5 & 25.5 & $1.45 \times 10^{-5}$ & $18.61 \pm 0.34$ & $8.02 \pm 0.12$ & $13.74 \pm 0.28$ & $1.27 \pm 0.08$ \\
\hline${ }^{33} \mathbf{S O}_{2}$ & $16_{4,12}-16_{3,13}$ & 339482.3 & 166.1 & $3.18 \times 10^{-4}$ & $2.72 \pm 0.31$ & $10.53 \pm 0.71$ & $12.33 \pm 1.53$ & $0.21 \pm 0.05$ \\
\hline $\mathrm{CN}^{\star}$ & $N=3-2, J=5 / 2-5 / 2$ & 339516.6 & 32.6 & $2.54 \times 10^{-5}$ & $3.15 \pm 0.50$ & $7.46 \pm 1.40$ & $17.27 \pm 3.49$ & $0.17 \pm 0.07$ \\
\hline${ }^{34} \mathbf{S O}^{\star}$ & $9_{8}-8_{7}$ & 339857.3 & 77.3 & $5.08 \times 10^{-4}$ & $70.69 \pm 0.33$ & $9.15 \pm 0.03$ & $14.43 \pm 0.08$ & $4.60 \pm 0.15$ \\
\hline $\mathbf{C N}^{\star}$ & $N=3-2, J=5 / 2-3 / 2$ & 340035.4 & 32.6 & $2.89 \times 10^{-4}$ & $51.50 \pm 1.01$ & $6.98 \pm 1.0$ & $17.38 \pm 0.68$ & $3.07 \pm 0.06$ \\
\hline $\mathrm{CH}_{3} \mathrm{OH}^{\star}$ & $2_{2}-3_{1} \mathrm{~A}^{+}$ & 340141.2 & 44.7 & $2.78 \times 10^{-5}$ & $4.38 \pm 0.35$ & $8.03 \pm 0.60$ & $14.91 \pm 1.39$ & $0.28 \pm 0.05$ \\
\hline $\mathrm{CN}^{\star \star}$ & $N=3-2, J=7 / 2-5 / 2$ & 340248.5 & 32.7 & $3.67 \times 10^{-4}$ & $51.14 \pm 0.99$ & & $13.25 \pm 1.42$ & $3.61 \pm 0.07$ \\
\hline${ }^{13} \mathrm{CH}_{3} \mathrm{OH}$ & $2_{2,1}-3_{1,2} \mathrm{~A}^{-}$ & 340313.9 & 44.6 & $2.82 \times 10^{-5}$ & $31.79 \pm 0.32$ & $8.21 \pm 0.07$ & $14.43 \pm 0.17$ & $2.07 \pm 0.08$ \\
\hline
\end{tabular}

Notes. The transitions marked with ${ }^{(\star)}$ were detected toward AFGL 2591 in the SLS survey (Van der Wiel 2011). The transitions marked with (^) have a non-Gaussian line profile. The FWHM line widths of these asymmetric lines are from a Gaussian fitting. Their intensity is obtained by integrating over the whole line and their $V_{\mathrm{LSR}}$ is the velocity at $T_{\text {peak }}$. The parameters without error bars are fixed parameters in the fit. ${ }^{(a)}$ May be blended with $\mathrm{SO}^{17} \mathrm{O}$. ${ }^{(b)}$ Probably blended with ${ }^{34} \mathrm{SO}_{2} 1_{2,14}-14_{1,13}$ at $358988 \mathrm{MHz} .{ }^{\left({ }^{c}\right)} \mathrm{HC}_{3} \mathrm{~N} 41-40$ is probable, but the line intensity is not consistent with its other three detected transitions. 
Table A.1. continued.

\begin{tabular}{|c|c|c|c|c|c|c|c|c|}
\hline Molecule & Transition & $\begin{array}{l}\text { Freq. } \\
(\mathrm{MHz})\end{array}$ & $\begin{array}{l}E_{\mathrm{up}} \\
(\mathrm{K})\end{array}$ & $\begin{array}{l}A_{\mathrm{ij}} \\
\left(\mathrm{s}^{-1}\right)\end{array}$ & $\begin{array}{l}\int T_{\mathrm{mb}} \mathrm{d} v \\
\left(\mathrm{~K} \mathrm{~km} \mathrm{~s}^{-1}\right)\end{array}$ & $\begin{array}{l}V_{\mathrm{LSR}} \\
\left(\mathrm{km} \mathrm{s}^{-1}\right)\end{array}$ & $\begin{array}{l}F W H M \\
\left(\mathrm{~km} \mathrm{~s}^{-1}\right)\end{array}$ & $\begin{array}{l}T_{\text {peak }} \\
(\mathrm{K})\end{array}$ \\
\hline $\mathrm{OCS}^{\star}$ & $28-27$ & 340449.3 & 237.0 & $1.15 \times 10^{-4}$ & $10.68 \pm 0.41$ & $10.38 \pm 0.29$ & $14.55 \pm 0.58$ & $0.69 \pm 0.08$ \\
\hline${ }^{33} \mathbf{S O}_{2}$ & $20_{1,19}-19_{2,18}$ & 340526.1 & 201.5 & $2.96 \times 10^{-4}$ & $4.61 \pm 0.32$ & $11.27 \pm 0.40$ & $11.24 \pm 0.86$ & $0.39 \pm 0.06$ \\
\hline $\mathbf{H C}^{18} \mathbf{O}^{+}$ & $4-3$ & 340630.7 & 40.9 & $3.11 \times 10^{-3}$ & $6.98 \pm 0.36$ & $7.58 \pm 0.29$ & $11.26 \pm 0.68$ & $0.58 \pm 0.07$ \\
\hline $\mathbf{S O}^{\star}$ & $7_{8}-6_{7}$ & 340714.2 & 81.2 & $4.99 \times 10^{-4}$ & $213.26 \pm 1.09$ & $7.49 \pm 0.04$ & $15.61 \pm 0.10$ & $12.83 \pm 0.52$ \\
\hline${ }^{33} \mathrm{SO}$ & $8_{8}-7_{7}$ & 340839.6 & 86.8 & $5.03 \times 10^{-4}$ & $22.64 \pm 0.26$ & $10.49 \pm 0.08$ & $13.84 \pm 0.18$ & $1.54 \pm 0.09$ \\
\hline $\mathbf{S O}_{2}$ & $21_{8,14}-22_{7,15}$ & 341275.5 & 369.1 & $6.86 \times 10^{-5}$ & $14.77 \pm 0.36$ & $11.60 \pm 0.15$ & $12.69 \pm 0.37$ & $1.09 \pm 0.10$ \\
\hline $\mathbf{H C S}^{+}$ & $8-7$ & 341350.2 & 73.7 & $8.35 \times 10^{-4}$ & $5.68 \pm 0.37$ & $9.40 \pm 0.46$ & $13.38 \pm 0.87$ & $0.40 \pm 0.08$ \\
\hline $\mathrm{CH}_{3} \mathrm{OH}^{\star}$ & $7_{1}-6_{1} \mathrm{~A}^{-}$ & 341415.6 & 80.1 & $1.71 \times 10^{-4}$ & $36.71 \pm 0.48$ & $10.62 \pm 0.14$ & $20.43 \pm 0.31$ & $1.69 \pm 0.19$ \\
\hline HCO & $4_{1,4}-3_{1,3}$ & 341671.6 & 73.8 & $3.46 \times 10^{-4}$ & $18.60 \pm 0.31$ & $7.78 \pm 0.14$ & $16.70 \pm 0.33$ & $1.05 \pm 0.09$ \\
\hline${ }^{33} \mathbf{S O}_{2}$ & $13_{2,12}-12_{1,11}$ & 341721.7 & 94.1 & $2.30 \times 10^{-4}$ & $10.70 \pm 1.45$ & $10.51 \pm 0.95$ & $17.67 \pm 2.28$ & $0.57 \pm 0.09$ \\
\hline $\mathrm{CH}_{3} \mathrm{CCH}$ & $20-19$ & 341741.0 & 172.2 & $1.39 \times 10^{-4}$ & $9.38 \pm 1.43$ & $9.82 \pm 0.86$ & $14.53 \pm 1.58$ & $0.61 \pm 0.09$ \\
\hline${ }^{34} \mathbf{S O}_{2}$ & $5_{3,3}-4_{2,2}$ & 342208.9 & 35.1 & $3.10 \times 10^{-4}$ & $15.29 \pm 0.51$ & $11.77 \pm 0.19$ & $11.83 \pm 0.46$ & $1.21 \pm 0.10$ \\
\hline${ }^{34} \mathbf{S O}_{2}$ & $20_{1,19}-19_{2,18}$ & 342231.6 & 198.5 & $3.06 \times 10^{-4}$ & $16.20 \pm 0.52$ & $11.60 \pm 0.21$ & $14.02 \pm 0.55$ & $1.09 \pm 0.10$ \\
\hline${ }^{34} \mathbf{S O}_{2}$ & $12_{4,8}-12_{3,9}$ & 342332.0 & 109.7 & $3.06 \times 10^{-4}$ & $15.07 \pm 0.47$ & $11.57 \pm 0.20$ & $12.77 \pm 0.46$ & $1.11 \pm 0.10$ \\
\hline $\mathrm{CH}_{3} \mathrm{OH}^{\star}$ & $13_{1}-13_{0} \mathrm{~A}^{\mp}$ & 342729.8 & 227.5 & $4.23 \times 10^{-4}$ & $9.95 \pm 0.38$ & $9.06 \pm 0.26$ & $13.71 \pm 0.57$ & $0.68 \pm 0.09$ \\
\hline $\mathbf{O}^{13} \mathbf{C}^{34} \mathbf{S}$ & $29-28$ & 342759.8 & 246.8 & $1.18 \times 10^{-4}$ & $17.47 \pm 0.35$ & $9.66 \pm 0.14$ & $13.45 \pm 0.34$ & $1.22 \pm 0.09$ \\
\hline $\mathbf{C S}^{\star}$ & $7-6$ & 342882.9 & 65.8 & $8.40 \times 10^{-4}$ & $155.79 \pm 0.66$ & $6.35 \pm 0.03$ & $14.21 \pm 0.08$ & $10.30 \pm 0.80$ \\
\hline $\mathrm{H}_{2} \mathrm{CS}$ & $10_{0,10}-9_{0,9}$ & 342946.4 & 90.6 & $6.08 \times 10^{-4}$ & $4.15 \pm 0.35$ & $9.11 \pm 0.52$ & $11.58 \pm 0.99$ & $0.34 \pm 0.06$ \\
\hline $\mathbf{H O}^{13} \mathrm{C}^{+}$ & $4-3$ & 342983.3 & 41.2 & $3.34 \times 10^{-3}$ & $2.49 \pm 0.35$ & $10.83 \pm 0.82$ & $11.91 \pm 2.29$ & $0.20 \pm 0.07$ \\
\hline${ }^{33} \mathrm{SO}$ & $9_{8}-8_{7}, F=\frac{17}{2}-\frac{15}{2}$ & 343087.3 & 78.0 & $5.09 \times 10^{-4}$ & $30.65 \pm 0.53$ & $9.66 \pm 0.12$ & $14.21 \pm 0.29$ & $2.03 \pm 0.10$ \\
\hline $\mathbf{H}_{2}^{13} \mathbf{C O}$ & $5_{15}-4_{14}$ & 343325.7 & 61.3 & $1.12 \times 10^{-3}$ & $8.76 \pm 0.58$ & $10.88 \pm 0.60$ & $18.27 \pm 1.50$ & $0.45 \pm 0.07$ \\
\hline $\mathrm{H}_{2} \mathrm{CS}$ & $10_{3,7}-9_{3,6}$ & 343414.1 & 209.1 & $5.56 \times 10^{-4}$ & $5.62 \pm 0.33$ & $12.12 \pm 0.48$ & $15.75 \pm 0.89$ & $0.33 \pm 0.09$ \\
\hline $\mathrm{H}_{2} \mathrm{CS}$ & $10_{2,8}-9_{2,7}$ & 343813.2 & 143.4 & $5.88 \times 10^{-4}$ & $2.56 \pm 0.41$ & $10.25 \pm 0.83$ & $11.91 \pm 2.68$ & $0.20 \pm 0.10$ \\
\hline $\mathbf{H C}^{15} \mathbf{N}^{\star}$ & $4-3$ & 344200.1 & 41.3 & $1.88 \times 10^{-3}$ & $13.85 \pm 0.46$ & $8.49 \pm 0.24$ & $14.51 \pm 0.56$ & $0.90 \pm 0.09$ \\
\hline${ }^{34} \mathbf{S O}_{2}$ & $10_{4,6}-10_{3,7}$ & 344245.3 & 88.5 & $2.96 \times 10^{-4}$ & $12.41 \pm 0.36$ & $10.85 \pm 0.17$ & $11.56 \pm 0.39$ & $1.01 \pm 0.07$ \\
\hline $\mathbf{S O}^{\star}$ & $88-77$ & 344310.6 & 87.5 & $5.19 \times 10^{-4}$ & $199.46 \pm 1.96$ & $7.39 \pm 0.07$ & $15.37 \pm 0.17$ & $12.19 \pm 0.50$ \\
\hline${ }^{34} \mathbf{S O}_{2}{ }^{\star}$ & $19_{1,19}-18_{0,18}$ & 344581.0 & 167.7 & $5.16 \times 10^{-4}$ & $20.86 \pm 0.43$ & $11.35 \pm 0.12$ & $12.20 \pm 0.30$ & $1.61 \pm 0.15$ \\
\hline${ }^{34} \mathbf{S O}_{2}{ }^{\star}$ & $13_{4,10}-13_{3,11}$ & 344807.9 & 121.6 & $3.17 \times 10^{-4}$ & $14.45 \pm 0.54$ & $11.54 \pm 0.25$ & $13.69 \pm 0.64$ & $0.99 \pm 0.12$ \\
\hline${ }^{34} \mathbf{S O}_{2}$ & $15_{4,12}-15_{3,13}$ & 344987.6 & 148.3 & $3.27 \times 10^{-4}$ & $26.22 \pm 0.64$ & $6.18 \pm 0.22$ & $17.92 \pm 0.49$ & $1.37 \pm 0.14$ \\
\hline${ }^{33} \mathrm{SO}_{2}$ & $14_{4,10}-4_{3,11}$ & 345134.4 & 137.1 & $3.19 \times 10^{-6}$ & $7.92 \pm 1.14$ & $12.86 \pm 0.93$ & $15.36 \pm 2.61$ & $0.48 \pm 0.12$ \\
\hline $\mathbf{S O}_{2}$ & $5_{5,1}-6_{4,2}$ & 345149.0 & 75.1 & $9.81 \times 10^{-6}$ & $8.51 \pm 1.20$ & $11.36 \pm 0.41$ & $9.70 \pm 1.13$ & $0.82 \pm 0.12$ \\
\hline${ }^{34} \mathrm{SO}_{2}$ & $8_{4,4}-8_{3,5}$ & 345168.7 & 71.0 & $2.75 \times 10^{-4}$ & $12.42 \pm 0.68$ & $12.25 \pm 0.28$ & $11.39 \pm 0.83$ & $1.02 \pm 0.12$ \\
\hline${ }^{34} \mathbf{S O}_{2}$ & $9_{4,6}-9_{3,7}$ & 345285.6 & 79.3 & $2.88 \times 10^{-4}$ & $13.67 \pm 0.66$ & $11.93 \pm 0.25$ & $10.22 \pm 0.56$ & $1.26 \pm 0.17$ \\
\hline $\mathbf{H}^{13} \mathbf{C N}^{\star}$ & $4-3$ & 345339.8 & 41.4 & $1.90 \times 10^{-3}$ & $110.90 \pm 0.72$ & $8.59 \pm 0.05$ & $15.70 \pm 0.12$ & $6.64 \pm 0.46$ \\
\hline $\mathrm{SO}_{2}$ & $26_{9,17}-27_{8,20}$ & 345449.0 & 521.0 & $7.63 \times 10^{-5}$ & $11.82 \pm 0.65$ & $11.12 \pm 0.35$ & $13.25 \pm 0.90$ & $0.84 \pm 0.17$ \\
\hline${ }^{34} \mathrm{SO}_{2}{ }^{\star}$ & $7_{4,4}-7_{3,5}$ & 345519.7 & 63.7 & $2.59 \times 10^{-4}$ & $14.84 \pm 0.63$ & $11.70 \pm 0.24$ & $11.80 \pm 0.62$ & $1.18 \pm 0.13$ \\
\hline${ }^{34} \mathbf{S O}_{2}$ & $6_{4,2}-6_{3,3}$ & 345553.1 & 57.3 & $2.35 \times 10^{-4}$ & $11.10 \pm 2.13$ & $12.08 \pm 0.96$ & $10.04 \pm 2.15$ & $1.04 \pm 0.13$ \\
\hline${ }^{33} \mathbf{S O}_{2}$ & $19_{1,19}-18_{0,18}$ & 345584.7 & 170.2 & $5.19 \times 10^{-4}$ & $4.22 \pm 0.60$ & $12.85 \pm 0.53$ & $7.71 \pm 1.28$ & $0.51 \pm 0.11$ \\
\hline $\mathbf{H C}_{3} \mathbf{N}$ & $38-37$ & 345609.0 & 323.5 & $3.30 \times 10^{-3}$ & $6.83 \pm 0.41$ & $12.06 \pm 0.35$ & $13.19 \pm 1.07$ & $0.49 \pm 0.09$ \\
\hline${ }^{34} \mathbf{S O}_{2}$ & $5_{4,2}-5_{3,3}$ & 345651.3 & 51.8 & $1.96 \times 10^{-4}$ & $6.12 \pm 0.78$ & $11.99 \pm 0.51$ & $7.46 \pm 0.98$ & $0.77 \pm 0.11$ \\
\hline${ }^{34} \mathbf{S O}_{2}$ & $4_{4,0}-4_{3,1}$ & 345678.8 & 47.2 & $1.31 \times 10^{-4}$ & $3.38 \pm 0.39$ & $11.30 \pm 0.61$ & $10.38 \pm 1.30$ & $0.31 \pm 0.09$ \\
\hline $\mathrm{CO}^{\star}$ & $3-2$ & 345796.0 & 33.2 & $2.50 \times 10^{-6}$ & $1161.6 \pm 4.43$ & $2.77 \pm 0.001$ & $21.22 \pm 0.10$ & $51.42 \pm 7.3$ \\
\hline${ }^{34} \mathbf{S O}_{2}$ & $17_{4,14}-17_{3,15}$ & 345929.3 & 178.8 & $3.37 \times 10^{-4}$ & $9.96 \pm 0.53$ & $12.02 \pm 0.32$ & $12.24 \pm 0.77$ & $0.76 \pm 0.09$ \\
\hline $\mathbf{N S}^{\star}$ & $15 / 2-13 / 2$ & 346220.1 & 71.0 & $7.38 \times 10^{-4}$ & $7.35 \pm 0.50$ & $10.84 \pm 0.68$ & $20.31 \pm 1.54$ & $0.34 \pm 0.06$ \\
\hline $\mathbf{S} \mathbf{O}_{2}, v_{2}=1$ & $19_{1,19}-18_{0,18}$ & 346379.2 & 930.6 & $5.16 \times 10^{-4}$ & $9.82 \pm 0.41$ & $9.03 \pm 0.38$ & $18.64 \pm 0.91$ & $0.49 \pm 0.09$ \\
\hline $\mathbf{H C}_{3} \mathbf{N}, v_{7}=1$ & $J=38-37$ & 346455.7 & 645.1 & $3.31 \times 10^{-3}$ & $2.10 \pm 0.34$ & $13.99 \pm 0.96$ & $9.77 \pm 2.07$ & $0.20 \pm 0.07$ \\
\hline $\mathbf{S O}^{\star}$ & $9_{8}-8_{7}$ & 346528.5 & 78.8 & $5.38 \times 10^{-4}$ & $231.50 \pm 0.69$ & $7.63 \pm 0.02$ & $16.69 \pm 0.06$ & $13.03 \pm 0.68$ \\
\hline${ }^{33} \mathrm{SO}_{2}$ & $5_{3,3}-4_{2,2}$ & 346589.8 & 36.0 & $1.76 \times 10^{-5}$ & $6.41 \pm 0.33$ & $10.91 \pm 0.30$ & $11.76 \pm 0.71$ & $0.51 \pm 0.06$ \\
\hline $\mathrm{SO}_{2}^{\star}$ & $19_{1,19}-18_{0,18}$ & 346652.2 & 168.1 & $5.22 \times 10^{-4}$ & $96.43 \pm 0.32$ & $9.42 \pm 0.03$ & $15.47 \pm 0.06$ & $5.85 \pm 0.14$ \\
\hline $\mathbf{H C}_{3} \mathbf{N} v_{7}=1$ & $J=38-37$ & 346949.1 & 645.6 & $3.32 \times 10^{-3}$ & $1.55 \pm 0.30$ & $13.93 \pm 0.73$ & $6.88 \pm 1.66$ & $0.21 \pm 0.09$ \\
\hline $\mathbf{H}^{13} \mathbf{C O}^{+\star}$ & $4-3$ & 346998.3 & 41.6 & $3.29 \times 10^{-3}$ & $50.66 \pm 0.41$ & $6.58 \pm 0.05$ & $11.86 \pm 0.12$ & $4.01 \pm 0.19$ \\
\hline $\mathrm{SiO}$ & $8-7$ & 347330.6 & 75.0 & $2.20 \times 10^{-3}$ & $68.67 \pm 0.66$ & $7.91 \pm 0.08$ & $16.98 \pm 0.20$ & $3.80 \pm 0.16$ \\
\hline${ }^{34} \mathrm{SO}_{2}$ & $28_{2,26}-28_{1,27}$ & 347483.1 & 391.2 & $2.65 \times 10^{-4}$ & $5.01 \pm 0.37$ & $11.48 \pm 0.45$ & $11.89 \pm 1.03$ & $0.40 \pm 0.07$ \\
\hline $\mathbf{S O}^{+}$ & $8-7$ & 347740.0 & 70.1 & $2.28 \times 10^{-4}$ & $19.44 \pm 0.41$ & $8.83 \pm 0.15$ & $13.87 \pm 0.33$ & $1.32 \pm 0.09$ \\
\hline $\mathbf{S O}_{2}, v_{2}=1$ & $13_{2,12}-12_{1,11}$ & 347991.8 & 854.3 & $2.41 \times 10^{-4}$ & $4.48 \pm 0.47$ & $9.98 \pm 0.88$ & $16.43 \pm 1.81$ & $0.26 \pm 0.08$ \\
\hline${ }^{34} \mathbf{S O}_{2}$ & $19_{4,16}-19_{3,17}$ & 348117.5 & 212.9 & $3.50 \times 10^{-4}$ & $28.62 \pm 0.38$ & $11.22 \pm 0.09$ & $14.24 \pm 0.22$ & $1.89 \pm 0.10$ \\
\hline $\mathbf{H C}^{17} \mathbf{O}^{+}$ & $4-3$ & 348211.2 & 41.8 & $3.32 \times 10^{-3}$ & $1.64 \pm 0.33$ & $8.75 \pm 1.12$ & $10.73 \pm 2.59$ & $0.14 \pm 0.05$ \\
\hline $\mathbf{H N}^{13} \mathbf{C}$ & $4-3$ & 348340.8 & 41.8 & $2.03 \times 10^{-3}$ & $5.66 \pm 1.05$ & $11.00 \pm 1.00$ & $11.88 \pm 1.00$ & $0.45 \pm 0.11$ \\
\hline $\mathbf{S O}_{2}{ }^{\star}$ & $24_{2,22}-23_{3,21}$ & 348387.8 & 292.7 & $1.91 \times 10^{-4}$ & $48.45 \pm 1.14$ & $10.12 \pm 1.00$ & $13.99 \pm 1.00$ & $3.25 \pm 0.10$ \\
\hline${ }^{33} \mathbf{S O}_{2}$ & $12_{4,8}-12_{3,9}$ & 348490.9 & 111.9 & $2.96 \times 10^{-6}$ & $6.28 \pm 0.78$ & $8.90 \pm 1.17$ & $18.08 \pm 3.31$ & $0.33 \pm 0.10$ \\
\hline $\mathbf{o}-\mathrm{H}_{2} \mathrm{CS}^{\star}$ & $10_{1,9}-9_{1,8}$ & 348534.4 & 105.2 & $6.32 \times 10^{-4}$ & $13.13 \pm 0.62$ & $9.32 \pm 0.36$ & $15.55 \pm 0.86$ & $0.79 \pm 0.10$ \\
\hline $\mathbf{C}_{2} \mathbf{H}^{\star}$ & $4_{9 / 2}-3_{5 / 2}, F=4-3$ & 349108.5 & 41.9 & $5.76 \times 10^{-8}$ & $9.05 \pm 0.46$ & $9.59 \pm 0.37$ & $14.35 \pm 0.80$ & $0.59 \pm 0.08$ \\
\hline $\mathbf{S O}^{18} \mathrm{O}$ & $15_{4,12}-15_{3,13}$ & 349209.6 & 149.8 & $3.37 \times 10^{-4}$ & $2.84 \pm 0.62$ & $10.65 \pm 1.58$ & $12.26 \pm 3.04$ & $0.22 \pm 0.09$ \\
\hline
\end{tabular}


Table A.1. continued.

\begin{tabular}{|c|c|c|c|c|c|c|c|c|}
\hline Molecule & Transition & $\begin{array}{l}\text { Freq. } \\
(\mathrm{MHz})\end{array}$ & $\begin{array}{l}E_{\text {up }} \\
(\mathrm{K})\end{array}$ & $\begin{array}{l}A_{\mathrm{ij}} \\
\left(\mathrm{s}^{-1}\right)\end{array}$ & $\begin{array}{l}\int T_{\mathrm{mb}} \mathrm{d} v \\
\left(\mathrm{~K} \mathrm{~km} \mathrm{~s}^{-1}\right)\end{array}$ & $\begin{array}{l}V_{\mathrm{LSR}} \\
\left(\mathrm{km} \mathrm{s}^{-1}\right)\end{array}$ & $\begin{array}{l}F W H M \\
\left(\mathrm{~km} \mathrm{~s}^{-1}\right)\end{array}$ & $\begin{array}{l}T_{\text {peak }} \\
(\mathrm{K})\end{array}$ \\
\hline $\mathbf{S O}^{18} \mathrm{O}$ & $13_{4,10}-13_{3,11}$ & 349224.9 & 123.3 & $3.27 \times 10^{-4}$ & $2.44 \pm 0.53$ & $11.95 \pm 0.28$ & $4.76 \pm 1.27$ & $0.48 \pm 0.09$ \\
\hline $\mathbf{C}_{2} \mathbf{H}^{\star}$ & $4_{9 / 2}-3_{7 / 2}$ & 349338.3 & 41.9 & $1.28 \times 10^{-4}$ & $33.15 \pm 0.46$ & $8.49 \pm 0.10$ & $13.97 \pm 0.22$ & $2.23 \pm 0.17$ \\
\hline $\mathbf{C}_{2} \mathbf{H}^{\star}$ & $4_{7 / 2}-3_{5 / 2}$ & 349400.7 & 41.9 & $1.20 \times 10^{-4}$ & $28.34 \pm 1.14$ & $10.32 \pm 0.24$ & $14.04 \pm 0.46$ & $1.90 \pm 0.10$ \\
\hline $\mathrm{CH}_{3} \mathrm{CN}^{\star}$ & $19_{2}-18_{2}$ & 349426.8 & 196.3 & $3.68 \times 10^{-3}$ & $5.91 \pm 3.13$ & $10.57 \pm 2.57$ & $19.45 \pm 12.17$ & $0.29 \pm 0.10$ \\
\hline $\mathrm{CH}_{3} \mathrm{CN}^{\star}$ & $19_{0}-18_{0}$ & 349453.7 & 167.7 & $3.72 \times 10^{-3}$ & $9.74 \pm 1.63$ & $12.05 \pm 1.12$ & $15.03 \pm 1.74$ & $0.61 \pm 0.10$ \\
\hline${ }^{33} \mathbf{S O}_{2}$ & $10_{4,6}-10_{3,7}$ & 350302.4 & 90.4 & $4.02 \times 10^{-6}$ & $2.87 \pm 0.34$ & $10.39 \pm 0.73$ & $11.41 \pm 1.29$ & $0.24 \pm 0.08$ \\
\hline $\mathrm{HNCO}^{\star}$ & $16_{1,16}-15_{1,15}$ & 350333.1 & 186.2 & $5.97 \times 10^{-4}$ & $5.82 \pm 0.32$ & $12.30 \pm 0.26$ & $9.89 \pm 0.64$ & $0.55 \pm 0.08$ \\
\hline $\mathrm{NO}^{\star}$ & $7 / 2-5 / 2^{+}$ & 350694.8 & 36.1 & $4.81 \times 10^{-6}$ & $35.27 \pm 0.44$ & $12.03 \pm 0.09$ & $14.78 \pm 0.21$ & $2.24 \pm 0.06$ \\
\hline${ }^{33} \mathbf{S O}_{2}$ & $13_{4,10}-13_{3,11}$ & 350787.0 & 124.0 & $2.64 \times 10^{-6}$ & $2.84 \pm 0.36$ & $11.42 \pm 0.89$ & $14.02 \pm 2.08$ & $0.19 \pm 0.08$ \\
\hline $\mathrm{SO}_{2}^{\star}$ & $10_{6,4}-11_{5,7}$ & 350862.8 & 138.8 & $4.40 \times 10^{-5}$ & $24.44 \pm 0.51$ & $11.46 \pm 0.13$ & $12.58 \pm 0.30$ & $1.83 \pm 0.12$ \\
\hline $\mathrm{CH}_{3} \mathrm{OH}^{\star}$ & $1_{1}-0_{0} A^{+}$ & 350905.1 & 16.8 & $3.31 \times 10^{-4}$ & $28.53 \pm 0.52$ & $5.82 \pm 0.11$ & $12.22 \pm 0.28$ & $2.19 \pm 0.24$ \\
\hline $\mathbf{S O}^{17} \mathrm{O}$ & $12_{4,8}-12_{3,9}$ & 350993.2 & 112.1 & $3.27 \times 10^{-4}$ & $5.55 \pm 0.54$ & $11.15 \pm 0.46$ & $9.97 \pm 1.17$ & $0.52 \pm 0.12$ \\
\hline $\mathrm{NO}^{\star}$ & $7 / 2-5 / 2^{-}$ & 351049.0 & 36.1 & $4.99 \times 10^{-6}$ & $35.03 \pm 0.61$ & $9.79 \pm 0.14$ & $16.80 \pm 0.34$ & $1.96 \pm 0.13$ \\
\hline${ }^{33} \mathbf{S O}_{2}$ & $8_{4,4}-8_{3,5}$ & 351177.4 & 72.7 & $5.65 \times 10^{-6}$ & $4.14 \pm 0.43$ & $12.48 \pm 0.38$ & $8.01 \pm 1.05$ & $0.49 \pm 0.09$ \\
\hline $\mathrm{SO}_{2}{ }^{\star}$ & $5_{3,3}-4_{2,2}$ & 351257.2 & 35.9 & $3.36 \times 10^{-4}$ & $42.96 \pm 0.59$ & $9.27 \pm 0.11$ & $16.27 \pm 0.28$ & $2.48 \pm 0.13$ \\
\hline${ }^{33} \mathrm{SO}_{2}$ & $7_{4,4}-7_{3,5}$ & 351508.5 & 65.2 & $6.76 \times 10^{-6}$ & $3.09 \pm 0.34$ & $11.35 \pm 0.47$ & $8.20 \pm 0.96$ & $0.35 \pm 0.09$ \\
\hline${ }^{33} \mathbf{S O}_{2}$ & $6_{4,2}-6_{3,3}$ & 351542.1 & 58.7 & $8.06 \times 10^{-6}$ & $8.37 \pm 0.55$ & $13.22 \pm 0.61$ & $18.63 \pm 1.30$ & $0.42 \pm 0.09$ \\
\hline HNCO & $16_{0,16}-15_{0,15}$ & 351633.3 & 143.5 & $6.13 \times 10^{-4}$ & $20.41 \pm 0.79$ & $12.36 \pm 0.24$ & $12.78 \pm 0.57$ & $1.50 \pm 0.12$ \\
\hline${ }^{33} \mathbf{S O}_{2}$ & $4_{4,0}-4_{3,1}$ & 351661.8 & 48.5 & $1.18 \times 10^{-5}$ & $3.39 \pm 0.53$ & $9.73 \pm 0.85$ & $10.67 \pm 1.90$ & $0.30 \pm 0.11$ \\
\hline${ }^{33} \mathbf{S O}_{2}$ & $17_{4,14}-17_{3,15}$ & 351743.7 & 181.9 & $1.78 \times 10^{-6}$ & $5.17 \pm 0.53$ & $13.39 \pm 0.56$ & $11.22 \pm 1.08$ & $0.43 \pm 0.18$ \\
\hline $\mathbf{o}-\mathrm{H}_{2} \mathrm{CO}^{\star}$ & $5_{1,5}-4_{1,4}$ & 351768.6 & 62.5 & $1.20 \times 10^{-3}$ & $104.71 \pm 1.35$ & $6.54 \pm 0.08$ & $13.75 \pm 0.22$ & $7.15 \pm 0.65$ \\
\hline $\mathrm{SO}_{2}^{\star}$ & $14_{4,10}-14_{3,11}$ & 351873.9 & 135.9 & $3.43 \times 10^{-4}$ & $63.49 \pm 0.71$ & $10.17 \pm 0.08$ & $14.94 \pm 0.19$ & $3.99 \pm 0.17$ \\
\hline${ }^{34} \mathbf{S O}_{2}$ & $21_{4,18}-21_{3,19}$ & 352082.9 & 250.8 & $3.65 \times 10^{-4}$ & $8.53 \pm 0.59$ & $11.65 \pm 0.37$ & $11.33 \pm 0.96$ & $0.71 \pm 0.07$ \\
\hline $\mathrm{OCS}^{\star}$ & $29-28$ & 352599.6 & 253.9 & $1.28 \times 10^{-4}$ & $11.23 \pm 0.33$ & $10.91 \pm 0.20$ & $13.54 \pm 0.44$ & $0.78 \pm 0.08$ \\
\hline $\mathrm{HNCO}^{\star}$ & $16_{1,15}-15_{1,14}$ & 352897.6 & 187.2 & $6.10 \times 10^{-4}$ & $6.28 \pm 0.33$ & $11.71 \pm 0.27$ & $10.12 \pm 0.63$ & $0.58 \pm 0.07$ \\
\hline${ }^{34} \mathbf{S O}_{2}$ & $14_{7,7}-15_{6,10}$ & 353002.4 & 212.6 & $5.70 \times 10^{-5}$ & $1.18 \pm 0.20$ & $11.24 \pm 0.98$ & $10.59 \pm 1.51$ & $0.11 \pm 0.05$ \\
\hline $\mathbf{S O}^{18} \mathbf{O}$ & $26_{3,24}-26_{2,25}$ & 353195.8 & 342.7 & $2.94 \times 10^{-4}$ & $1.59 \pm 0.25$ & $12.54 \pm 0.89$ & $11.07 \pm 1.87$ & $0.13 \pm 0.03$ \\
\hline $\mathbf{H}$ & $26 \alpha$ & 353622.8 & & & $18.97 \pm 0.46$ & $11.63 \pm 0.44$ & $37.31 \pm 1.07$ & $0.48 \pm 0.06$ \\
\hline $\mathrm{CO}^{+a}$ & $N=3-2, F=\frac{5}{2}-\frac{3}{2}$ & 353741.3 & 33.9 & $2.06 \times 10^{-4}$ & $5.55 \pm 0.45$ & $12.11 \pm 0.82$ & $21.09 \pm 2.06$ & $0.25 \pm 0.06$ \\
\hline $\mathbf{H C N}, v_{2}=1^{\text {blend } / 1}$ & $4-3 \quad 22$ & 354460.4 & 1066.9 & $1.87 \times 10^{-3}$ & $9.78 \pm 0.95$ & $12.50 \pm 1.0$ & $15.00 \pm 1.00$ & $0.62 \pm 0.06$ \\
\hline $\mathbf{H C N}^{\text {blend } / 2 \star \star}$ & $4-3$ & 354505.5 & 42.5 & $2.05 \times 10^{-3}$ & $184.9 \pm 1.30$ & $1.32 \pm 1.0$ & $22.00 \pm 2.00$ & $8.55 \pm 0.06$ \\
\hline $\mathbf{S} \mathbf{O}_{2}, v_{2}=1$ & $16_{4,12}-16_{3,13}$ & 354800.0 & 927.8 & $3.60 \times 10^{-4}$ & $3.16 \pm 0.32$ & $10.73 \pm 0.91$ & $15.39 \pm 1.89$ & $0.19 \pm 0.08$ \\
\hline $\mathrm{SO}_{2}{ }^{\star}$ & $12_{4,8}-12_{3,9}$ & 355045.5 & 111.0 & $3.40 \times 10^{-4}$ & $49.46 \pm 0.32$ & $9.74 \pm 0.05$ & $15.04 \pm 0.12$ & $3.09 \pm 0.09$ \\
\hline $\mathbf{S O}_{2}$ & $17_{4,14}-18_{1,17}$ & 355186.5 & 180.1 & $2.62 \times 10^{-6}$ & $5.16 \pm 0.36$ & $9.08 \pm 0.57$ & $16.41 \pm 1.47$ & $0.30 \pm 0.05$ \\
\hline $\mathbf{H}^{15} \mathbf{N C}$ & $4-3$ & 355439.5 & 42.6 & $1.69 \times 10^{-3}$ & $1.25 \pm 0.23$ & $10.68 \pm 0.83$ & $8.36 \pm 1.71$ & $0.14 \pm 0.05$ \\
\hline $\mathbf{S}^{18} \mathbf{O}$ & $8_{9}-7_{8}$ & 355571.1 & 93.1 & $5.74 \times 10^{-4}$ & $8.35 \pm 0.26$ & $11.21 \pm 0.24$ & $16.00 \pm 0.58$ & $0.49 \pm 0.06$ \\
\hline $\mathrm{CH}_{3} \mathrm{OH}^{\star}$ & $13_{0,13}-12_{1,12} \mathrm{~A}^{+}$ & 355603.1 & 211.0 & $2.53 \times 10^{-4}$ & $9.52 \pm 0.27$ & $9.61 \pm 0.22$ & $15.04 \pm 0.47$ & $0.59 \pm 0.06$ \\
\hline $\mathrm{CH}_{3} \mathrm{OH}^{\star}$ & $15_{1,14}-15_{0,15} \mathrm{~A}^{+}$ & 356007.2 & 295.3 & $4.60 \times 10^{-4}$ & $7.87 \pm 0.62$ & $8.70 \pm 0.58$ & $14.19 \pm 1.13$ & $0.52 \pm 0.10$ \\
\hline $\mathbf{S O}_{2}$ & $15_{7,9}-16_{6,10}$ & 356040.6 & 230.4 & $6.40 \times 10^{-5}$ & $23.58 \pm 0.67$ & $10.51 \pm 0.19$ & $13.27 \pm 0.45$ & $1.67 \pm 0.10$ \\
\hline${ }^{34} \mathrm{SO}_{2}$ & $25_{3,23}-25_{2,24}$ & 356222.2 & 320.0 & $2.97 \times 10^{-4}$ & $6.39 \pm 0.51$ & $10.72 \pm 0.45$ & $10.87 \pm 0.92$ & $0.55 \pm 0.08$ \\
\hline $\mathbf{H C N}, v_{2}=1$ & $J=4-3$ & 356255.6 & 1067.12 & $5.33 \times 10^{-3}$ & $11.42 \pm 0.71$ & $12.20 \pm 0.57$ & $20.00 \pm 1.61$ & $0.54 \pm 0.07$ \\
\hline $\mathrm{HCO}^{+\star}$ & $4-3$ & 356734.2 & 42.8 & $3.57 \times 10^{-3}$ & $503.55 \pm 0.55$ & $3.10 \pm 0.01$ & $27.35 \pm 0.03$ & $17.30 \pm 1.30$ \\
\hline${ }^{34} \mathbf{S O}_{2}{ }^{\star}$ & $20_{0,20}-19_{1,19}$ & 357102.2 & 184.8 & $5.81 \times 10^{-4}$ & $25.19 \pm 0.53$ & $11.25 \pm 0.14$ & $13.36 \pm 0.33$ & $1.77 \pm 0.14$ \\
\hline $\mathrm{SO}_{2}^{\star}$ & $13_{4,10}-13_{3,11}$ & 357165.4 & 123.0 & $3.51 \times 10^{-4}$ & $72.28 \pm 0.41$ & $9.24 \pm 0.04$ & $15.20 \pm 0.10$ & $4.47 \pm 0.15$ \\
\hline $\mathbf{S O}_{2}{ }^{\star}$ & $15_{4,12}-15_{3,13}$ & 357241.2 & 149.7 & $3.62 \times 10^{-4}$ & $67.49 \pm 0.68$ & $9.24 \pm 0.07$ & $14.70 \pm 0.17$ & $4.31 \pm 0.14$ \\
\hline $\mathrm{SO}_{2}{ }^{\star}$ & $11_{4,8}-11_{3,9}$ & 357387.6 & 100.0 & $3.38 \times 10^{-4}$ & $71.00 \pm 0.84$ & $9.85 \pm 0.09$ & $15.00 \pm 0.21$ & $4.45 \pm 0.13$ \\
\hline${ }^{34} \mathrm{SO}_{2}$ & $32_{5,27}-32_{4,28}$ & 357497.8 & 547.5 & $4.55 \times 10^{-4}$ & $2.34 \pm 0.24$ & $12.21 \pm 0.47$ & $9.23 \pm 1.22$ & $0.24 \pm 0.05$ \\
\hline $\mathbf{S O}_{2}{ }^{\star}$ & $8_{4,4}-8_{3,5}$ & 357581.4 & 72.4 & $3.06 \times 10^{-4}$ & $81.60 \pm 0.61$ & $9.32 \pm 0.06$ & $17.96 \pm 0.16$ & $4.27 \pm 0.24$ \\
\hline $\mathbf{S O}_{2}{ }^{\star}$ & $9_{4,6}-9_{3,7}$ & 357671.8 & 80.6 & $3.20 \times 10^{-4}$ & $86.02 \pm 0.30$ & $9.88 \pm 0.03$ & $16.93 \pm 0.07$ & $4.77 \pm 0.23$ \\
\hline $\mathbf{S O}_{2}^{\text {blend } / 1 \star}$ & $7_{4,4}-7_{3,5}$ & 357892.4 & 65.0 & $2.87 \times 10^{-4}$ & $78.52 \pm 2.36$ & $9.36 \pm 1.00$ & $15.65 \pm 1.00$ & $4.71 \pm 0.15$ \\
\hline $\mathbf{S O}_{2}^{\text {blend } / 2 \star}$ & $6_{4,2}-6_{3,3}$ & 357925.8 & 58.6 & $2.60 \times 10^{-4}$ & $74.40 \pm 0.36$ & $9.47 \pm 0.04$ & $15.64 \pm 0.09$ & $4.47 \pm 0.14$ \\
\hline $\mathbf{S O}_{2}$ blend $/ 3$ & $17_{4,14}-17_{3,15}$ & 357962.9 & 180.1 & $3.73 \times 10^{-4}$ & $69.61 \pm 0.38$ & $10.06 \pm 0.04$ & $15.86 \pm 0.10$ & $4.12 \pm 0.14$ \\
\hline $\mathbf{S O}_{2}{ }^{\text {blend}} / 4 \star$ & $5_{4,2}-5_{3,3}$ & 358013.2 & 53.1 & $2.18 \times 10^{-4}$ & $68.11 \pm 0.36$ & $9.71 \pm 0.01$ & $15.47 \pm 0.10$ & $4.14 \pm 0.14$ \\
\hline $\mathbf{S O}_{2}^{\text {blend } / 5 \star}$ & $4_{4,0}-4_{3,1}$ & 358037.9 & 48.5 & $1.45 \times 10^{-4}$ & $48.82 \pm 2.39$ & $9.68 \pm 1.00$ & $13.32 \pm 1.00$ & $3.44 \pm 0.14$ \\
\hline $\mathbf{S O}_{2}^{\star}$ & $20_{0,20}-19_{1,19}$ & 358215.6 & 185.3 & $5.83 \times 10^{-4}$ & $102.21 \pm 0.56$ & $9.29 \pm 0.04$ & $15.47 \pm 0.10$ & $6.21 \pm 0.17$ \\
\hline${ }^{34} \mathbf{S O}_{2}$ & $23_{4,20}-23_{3,21}$ & 358347.3 & 292.4 & $3.86 \times 10^{-4}$ & $9.32 \pm 0.30$ & $11.67 \pm 0.20$ & $12.70 \pm 0.48$ & $0.69 \pm 0.08$ \\
\hline $\mathrm{CH}_{3} \mathrm{OCH}_{3}$ & $5_{5,1,1}-4_{4,1,1}$ & 358454.0 & 48.8 & $2.94 \times 10^{-4}$ & $3.32 \pm 0.30$ & $8.56 \pm 0.68$ & $14.95 \pm 1.40$ & $0.21 \pm 0.05$ \\
\hline $\mathrm{CH}_{3} \mathrm{OH}^{\star}$ & $4_{1}-3_{0} E$ & 358605.8 & 44.3 & $1.32 \times 10^{-4}$ & $24.58 \pm 1.35$ & $7.66 \pm 0.38$ & $13.84 \pm 0.83$ & $1.67 \pm 0.14$ \\
\hline $\mathbf{S}^{18} \mathbf{O}$ & $9_{9}-8_{8}$ & 358645.7 & 99.3 & $5.92 \times 10^{-4}$ & $9.23 \pm 3.28$ & $9.38 \pm 2.27$ & $12.81 \pm 5.15$ & $0.68 \pm 0.05$ \\
\hline $\mathbf{C H}_{3} \mathbf{C C H}^{\text {blend } / 1}$ & $21_{3}-20_{3}$ & 358756.5 & 254.5 & $1.58 \times 10^{-4}$ & $3.63 \pm 0.18$ & $9.34 \pm 0.30$ & $12.03 \pm 0.66$ & $0.28 \pm 0.04$ \\
\hline $\mathbf{C H}_{3} \mathbf{C C H}^{\text {blend } / 2}$ & $21_{2}-20_{2}$ & 358790.6 & 218.3 & $1.60 \times 10^{-4}$ & $2.75 \pm 0.28$ & $8.37 \pm 0.67$ & $12.40 \pm 1.45$ & $0.21 \pm 0.06$ \\
\hline $\mathbf{C H}_{3} \mathbf{C C H}^{\text {blend } / 3}$ & $21_{0}-20_{0}$ & 358817.9 & 189.5 & $1.61 \times 10^{-4}$ & $7.92 \pm 0.43$ & $10.53 \pm 0.36$ & $14.48 \pm 0.93$ & $0.51 \pm 0.06$ \\
\hline $\mathbf{S O}^{17} \mathbf{O}^{b}$ & $15_{2,14}-14_{1,13}$ & 358986.2 & 119.9 & $2.84 \times 10^{-4}$ & $20.64 \pm 0.25$ & $9.95 \pm 0.08$ & $13.18 \pm 0.19$ & $1.47 \pm 0.10$ \\
\hline
\end{tabular}


Table A.1. continued.

\begin{tabular}{|c|c|c|c|c|c|c|c|c|}
\hline Molecule & Transition & $\begin{array}{l}\text { Freq. } \\
(\mathrm{MHz})\end{array}$ & $\begin{array}{l}E_{\text {up }} \\
(\mathrm{K})\end{array}$ & $\begin{array}{l}A_{\mathrm{ij}} \\
\left(\mathrm{s}^{-1}\right)\end{array}$ & $\begin{array}{l}\int T_{\mathrm{mb}} \mathrm{d} v \\
\left(\mathrm{~K} \mathrm{~km} \mathrm{~s}^{-1}\right)\end{array}$ & $\begin{array}{l}V_{\mathrm{LSR}} \\
\left(\mathrm{km} \mathrm{s}^{-1}\right)\end{array}$ & $\begin{array}{l}F W H M \\
\left(\mathrm{~km} \mathrm{~s}^{-1}\right)\end{array}$ & $\begin{array}{l}T_{\text {peak }} \\
(\mathrm{K})\end{array}$ \\
\hline $\mathbf{S O}_{2}{ }^{\star}$ & $25_{3,23}-25_{2,24}$ & 359151.2 & 320.9 & $3.10 \times 10^{-4}$ & $42.63 \pm 0.21$ & $10.57 \pm 0.04$ & $14.47 \pm 0.09$ & $2.77 \pm 0.08$ \\
\hline${ }^{34} \mathbf{S O}_{2}$ & $24_{2,22}-23_{3,21}$ & 359651.7 & 292.4 & $2.20 \times 10^{-4}$ & $6.76 \pm 0.34$ & $12.02 \pm 0.26$ & $11.01 \pm 0.71$ & $0.58 \pm 0.07$ \\
\hline $\mathbf{S O}_{2}{ }^{\star}$ & $19_{4,16}-19_{3,17}$ & 359770.7 & 214.3 & $3.85 \times 10^{-4}$ & $58.89 \pm 0.28$ & $10.13 \pm 0.03$ & $15.16 \pm 0.08$ & $3.65 \pm 0.10$ \\
\hline $\mathbf{S O}_{2}, v_{2}=1$ & $14_{4,10}-14_{3,11}$ & 360133.2 & 898.7 & $1.43 \times 10^{-4}$ & $4.42 \pm 0.37$ & $11.53 \pm 0.58$ & $13.44 \pm 1.23$ & $0.31 \pm 0.06$ \\
\hline $\begin{array}{l}\mathbf{S O}_{2}, \mathbf{O}_{2}-1 \\
\end{array}$ & $34_{5,29}-34_{4,30}$ & 360290.4 & 612.0 & $4.80 \times 10^{-4}$ & $18.63 \pm 0.32$ & $11.14 \pm 0.11$ & $13.93 \pm 0.28$ & $1.26 \pm 0.06$ \\
\hline $\mathbf{S}^{18} \mathbf{O}$ & $10_{9}-9_{8}$ & 360637.9 & 90.6 & $6.10 \times 10^{-4}$ & $11.30 \pm 0.52$ & $9.65 \pm 0.32$ & $13.98 \pm 0.71$ & $0.76 \pm 0.09$ \\
\hline $\mathrm{SO}_{2}{ }^{\star}$ & $20_{8,12}-21_{7,15}$ & 360721.8 & 349.8 & $7.72 \times 10^{-5}$ & $15.07 \pm 0.55$ & $11.51 \pm 0.22$ & $12.59 \pm 0.56$ & $1.12 \pm 0.12$ \\
\hline $\mathrm{CH}_{3} \mathrm{OH}^{\star}$ & $11_{0,11}-10_{1,9} E$ & 360848.9 & 166.0 & $1.21 \times 10^{-4}$ & $8.12 \pm 0.44$ & $8.42 \pm 0.42$ & $15.26 \pm 0.87$ & $0.50 \pm 0.08$ \\
\hline NO & $J=7 / 2-5 / 2$ & 360948.3 & 209.4 & $4.52 \times 10^{-6}$ & $15.30 \pm 0.55$ & $11.31 \pm 0.52$ & $29.18 \pm 1.19$ & $0.49 \pm 0.06$ \\
\hline $\mathrm{CH}_{3} \mathrm{OH}^{\star}$ & $8_{1}-7_{2} E$ & 361852.3 & 104.6 & $7.70 \times 10^{-5}$ & $11.25 \pm 0.38$ & $6.58 \pm 0.27$ & $16.60 \pm 0.68$ & $0.64 \pm 0.07$ \\
\hline $\mathbf{D C N}^{\star}$ & $5-4$ & 362045.8 & 52.1 & $2.25 \times 10^{-3}$ & $2.26 \pm 0.33$ & $6.99 \pm 0.75$ & $9.17 \pm 1.22$ & $0.23 \pm 0.09$ \\
\hline${ }^{34} \mathbf{S O}_{2}{ }^{\star}$ & $6_{3,3}-5_{2,4}$ & 362158.2 & 40.7 & $3.29 \times 10^{-4}$ & $19.93 \pm 0.27$ & $11.17 \pm 0.08$ & $12.88 \pm 0.21$ & $1.45 \pm 0.09$ \\
\hline${ }^{33} \mathrm{SO}_{2}$ & $15_{2,14}-14_{1,13}$ & 362487.6 & 120.8 & $2.98 \times 10^{-4}$ & $6.11 \pm 0.25$ & $10.16 \pm 0.26$ & $13.16 \pm 0.61$ & $0.44 \pm 0.04$ \\
\hline $\mathbf{H N C}, v_{2}=1$ & $4-3$ & 362554.4 & 709.3 & $2.15 \times 10^{-3}$ & $4.20 \pm 0.29$ & $12.65 \pm 0.35$ & $11.39 \pm 1.06$ & $0.35 \pm 0.05$ \\
\hline $\mathrm{HNC}^{\star}$ & $4-3$ & 362630.3 & 43.5 & $2.30 \times 10^{-3}$ & $52.96 \pm 0.56$ & $5.72 \pm 0.06$ & $12.93 \pm 0.18$ & $3.85 \pm 0.42$ \\
\hline p- $\mathrm{H}_{2} \mathrm{CO}^{\star}$ & $5_{0,5}-4_{0,4}$ & 362736.0 & 52.3 & $1.37 \times 10^{-3}$ & $60.08 \pm 0.26$ & $6.91 \pm 0.03$ & $12.45 \pm 0.06$ & $4.53 \pm 0.25$ \\
\hline${ }^{34} \mathbf{S O}_{2}$ & $23_{2,22}-23_{1,23}$ & 362834.1 & 259.2 & $1.77 \times 10^{-4}$ & $6.75 \pm 0.31$ & $11.91 \pm 0.26$ & $11.59 \pm 0.65$ & $0.55 \pm 0.06$ \\
\hline $\mathrm{SO}_{2}{ }^{\star}$ & $21_{4,18}-21_{3,19}$ & 363159.3 & 252.1 & $4.00 \times 10^{-4}$ & $50.66 \pm 0.33$ & $10.33 \pm 0.05$ & $15.12 \pm 0.12$ & $3.15 \pm 0.10$ \\
\hline${ }^{33} \mathrm{SO}_{2}$ & $23_{4,20}-23_{3,21}$ & 363286.4 & 297.1 & $4.01 \times 10^{-4}$ & $6.25 \pm 0.35$ & $9.08 \pm 0.35$ & $13.79 \pm 1.02$ & $0.43 \pm 0.07$ \\
\hline $\mathrm{CH}_{3} \mathrm{OH}^{\star}$ & $16_{1}-16_{0} A^{\mp}$ & 363440.4 & 332.6 & $2.40 \times 10^{-4}$ & $6.63 \pm 0.39$ & $9.74 \pm 0.46$ & $15.26 \pm 0.94$ & $0.41 \pm 0.06$ \\
\hline $\mathrm{CH}_{3} \mathrm{OH}^{\star}$ & $7_{2}-6_{1} E$ & 363739.8 & 87.3 & $1.70 \times 10^{-4}$ & $17.91 \pm 0.26$ & $8.36 \pm 0.10$ & $13.80 \pm 0.22$ & $1.22 \pm 0.07$ \\
\hline $\mathbf{H C}_{3} \mathbf{N}$ & $40-39$ & 363785.4 & 358.0 & $3.85 \times 10^{-3}$ & $3.67 \pm 0.22$ & $12.39 \pm 0.28$ & $9.71 \pm 0.70$ & $0.35 \pm 0.05$ \\
\hline $\mathbf{S O}_{2}$ & $24_{1,23}-24_{0,24}$ & 363890.9 & 280.5 & $1.78 \times 10^{-4}$ & $23.45 \pm 0.24$ & $10.42 \pm 0.07$ & $14.59 \pm 0.18$ & $1.51 \pm 0.07$ \\
\hline $\mathrm{SO}_{2}^{\star}$ & $23_{2,22}-23_{1,23}$ & 363925.8 & 259.9 & $1.83 \times 10^{-4}$ & $27.90 \pm 0.34$ & $10.33 \pm 0.08$ & $15.27 \pm 0.22$ & $1.72 \pm 0.07$ \\
\hline p- $\mathbf{H}_{2} \mathrm{CO}^{\star}$ & $5_{2,4}-4_{2,3}$ & 363945.9 & 99.5 & $1.16 \times 10^{-3}$ & $25.65 \pm 0.28$ & $7.73 \pm 0.07$ & $12.59 \pm 0.15$ & $1.91 \pm 0.07$ \\
\hline p- $\mathrm{H}_{2} \mathrm{CO}$ & $5_{4,2}-4_{4,1}$ & 364103.2 & 240.7 & $4.99 \times 10^{-4}$ & $11.15 \pm 0.28$ & $8.12 \pm 0.17$ & $13.31 \pm 0.39$ & $0.79 \pm 0.06$ \\
\hline $\mathbf{o}-\mathrm{H}_{2} \mathrm{CO}^{\star}$ & $5_{3,3}-4_{3,2}$ & 364275.1 & 158.4 & $8.88 \times 10^{-4}$ & $39.52 \pm 0.32$ & $7.79 \pm 0.07$ & 12.00 & $3.09 \pm 0.14$ \\
\hline $\mathbf{o}-\mathbf{H}_{2} \mathrm{CO}^{\star}$ & $5_{3,2}-4_{3,1}$ & 364288.9 & 158.4 & $8.88 \times 10^{-4}$ & $39.70 \pm 0.38$ & $7.22 \pm 0.08$ & 12.00 & $3.11 \pm 0.14$ \\
\hline Atmospheric & & 364403.7 & & & & & & \\
\hline $\mathbf{H C}_{3} \mathbf{N} v_{7}=1$ & $J=40-39$ & 364676.3 & 679.7 & $3.86 \times 10^{-3}$ & $3.05 \pm 0.37$ & $15.90 \pm 1.10$ & $18.08 \pm 2.55$ & $0.16 \pm 0.06$ \\
\hline $\mathbf{O C S}^{\star}$ & $30-29$ & 364749.0 & 271.4 & $1.42 \times 10^{-4}$ & $8.45 \pm 0.31$ & $9.89 \pm 0.27$ & $14.07 \pm 0.56$ & $0.56 \pm 0.07$ \\
\hline $\mathbf{H}_{3} \mathbf{O}^{+}$ & $3_{2,1}-2_{2,0}$ & 364797.4 & 139.7 & $2.78 \times 10^{-4}$ & $17.88 \pm 0.35$ & $8.58 \pm 0.14$ & $14.41 \pm 0.30$ & $1.17 \pm 0.09$ \\
\hline $\mathbf{S O}_{2}$ & $25_{9,17}-26_{8,18}$ & 364950.1 & 497.1 & $8.70 \times 10^{-5}$ & $10.11 \pm 0.26$ & $12.12 \pm 0.14$ & $11.08 \pm 0.34$ & $0.86 \pm 0.08$ \\
\hline $\mathbf{C H}_{3} \mathbf{O H}, v_{t}=1$ & $5_{3}-5_{2} E$ & 364986.8 & 452.1 & $3.76 \times 10^{-5}$ & $3.59 \pm 0.24$ & $6.02 \pm 0.36$ & $10.52 \pm 0.80$ & $0.32 \pm 0.06$ \\
\hline HNC, $v_{2}=1$ & $4-3$ & 365147.5 & 709.6 & $2.20 \times 10^{-3}$ & $3.79 \pm 0.60$ & $13.27 \pm 0.21$ & $7.70 \pm 1.42$ & $0.46 \pm 0.07$ \\
\hline $\mathbf{H C}_{3} \mathbf{N} v_{7}=1$ & & 365195.2 & 680.2 & $3.88 \times 10^{-3}$ & $3.00 \pm 0.28$ & $13.77 \pm 0.52$ & $10.33 \pm 1.05$ & $0.27 \pm 0.09$ \\
\hline $\mathbf{p}-\mathrm{H}_{2} \mathbf{C O}^{\star}$ & $5_{2,3}-4_{2,2}$ & 365363.4 & 99.7 & $1.18 \times 10^{-3}$ & $44.27 \pm 0.74$ & $7.73 \pm 0.12$ & $14.23 \pm 0.28$ & $2.92 \pm 0.16$ \\
\hline $\mathrm{H}_{2} \mathbf{C N}$ & $5_{0,5}-4_{0,4}$ & 365443.6 & 52.7 & $4.15 \times 10^{-5}$ & $2.98 \pm 0.31$ & $10.05 \pm 0.49$ & $9.13 \pm 1.07$ & $0.31 \pm 0.06$ \\
\hline $\mathbf{H}_{2} \mathbf{C}^{34} \mathbf{S}$ & $11_{1,11}-10_{1,10}$ & 365613.4 & 118.5 & $7.33 \times 10^{-4}$ & $4.32 \pm 0.37$ & $9.25 \pm 0.51$ & $11.83 \pm 1.25$ & $0.34 \pm 0.07$ \\
\hline $\mathrm{CH}_{3} \mathrm{COCH}_{3}$ & $27_{11,16,0}-26_{12,15,1}$ & 365696.2 & 281.7 & $1.20 \times 10^{-3}$ & $2.97 \pm 0.41$ & $9.46 \pm 0.91$ & $13.63 \pm 2.40$ & $0.20 \pm 0.06$ \\
\hline${ }^{34} \mathbf{S O}_{2}$ & $24_{1,23}-24_{0,24}$ & 365794.6 & 279.9 & $1.75 \times 10^{-4}$ & $4.56 \pm 0.45$ & $13.44 \pm 0.26$ & $7.54 \pm 0.68$ & $0.57 \pm 0.07$ \\
\hline U-line & & 365811.5 & & & $6.31 \pm 0.84$ & $10.91 \pm 1.14$ & $19.03 \pm 3.20$ & $0.31 \pm 0.07$ \\
\hline $\mathbf{S} \mathbf{O}_{2}, v_{2}=1$ & $9_{4,6}-9_{3,7}$ & 365904.5 & 842.5 & $3.39 \times 10^{-4}$ & $3.42 \pm 0.53$ & $11.84 \pm 0.91$ & $11.60 \pm 2.68$ & $0.28 \pm 0.06$ \\
\hline $\mathbf{S O}_{2}, v_{2}=1^{\text {blend } / 1}$ & $7_{4,4}-7_{3,5}$ & 366125.8 & 826.6 & $3.05 \times 10^{-4}$ & $3.04 \pm 0.36$ & $12.82 \pm 0.71$ & 10.00 & $0.29 \pm 0.07$ \\
\hline $\mathbf{S O}_{2}, v_{2}=1^{\text {blend } / 2}$ & $17_{4,14}-17_{3,15}$ & 366145.1 & 943.8 & $3.95 \times 10^{-4}$ & $4.28 \pm 0.32$ & $14.10 \pm 0.50$ & 10.00 & $0.40 \pm 0.07$ \\
\hline $\mathbf{S O}_{2}, v_{2}=1^{\text {blend } / 3}$ & $6_{4,2}-6_{3,3}$ & 366159.5 & 820.0 & $2.76 \times 10^{-4}$ & $3.68 \pm 0.34$ & $13.16 \pm 0.67$ & 10.00 & $0.35 \pm 0.07$ \\
\hline $\mathrm{SO}_{2}{ }^{\star}$ & $15_{2,14}-14_{1,13}$ & 366214.5 & 119.3 & $3.04 \times 10^{-4}$ & $91.77 \pm 1.56$ & $9.43 \pm 0.13$ & $15.80 \pm 0.32$ & $5.46 \pm 0.15$ \\
\hline $\mathrm{H}_{2}^{13} \mathrm{CO}$ & $5_{1,4}-4_{1,3}$ & 366270.2 & 64.6 & $1.36 \times 10^{-3}$ & $6.96 \pm 0.77$ & $10.14 \pm 1.06$ & 14.00 & $0.47 \pm 0.08$ \\
\hline${ }^{33} \mathbf{S O}_{2}$ & $6,3-5_{2,4}$ & 366521.9 & 41.6 & $1.31 \times 10^{-5}$ & $7.52 \pm 0.33$ & $11.61 \pm 0.25$ & $11.79 \pm 0.64$ & $0.60 \pm 0.07$ \\
\hline $\mathrm{H}_{2} \mathrm{CN}$ & $5_{2,4}-4_{2,3}$ & 366637.0 & 100.4 & $9.42 \times 10^{-5}$ & $2.19 \pm 0.32$ & $9.06 \pm 1.04$ & 10.00 & $0.21 \pm 0.06$ \\
\hline $\mathbf{H}_{2} \mathbf{C N}$ & $5_{2,4}-4_{2,3}$ & 366655.4 & 100.4 & $7.69 \times 10^{-5}$ & $2.96 \pm 0.33$ & $8.36 \pm 0.80$ & 10.00 & $0.28 \pm 0.06$ \\
\hline Atmospheric & & 366790.0 & & & & & & \\
\hline${ }^{34} \mathrm{SO}_{2}$ & $25_{4,22}-25_{3,23}$ & 367369.3 & 337.6 & $4.14 \times 10^{-4}$ & $8.97 \pm 0.37$ & $11.97 \pm 0.26$ & $12.69 \pm 0.60$ & $0.66 \pm 0.09$ \\
\hline $\mathbf{o}-\mathbf{H}_{2} \mathbf{S}^{\text {blend } / 1 \star}$ & $3_{2,1}-3_{1,2}$ & 369101.4 & 154.5 & $1.90 \times 10^{-4}$ & $145.64 \pm 0.92$ & $8.54 \pm 0.04$ & $15.67 \pm 0.12$ & $8.73 \pm 0.43$ \\
\hline $\mathbf{p}-\mathbf{H}_{2} \mathbf{S}^{\text {blend } / 2}$ & $4_{3,1}-4_{2,2}$ & 369126.9 & 262.8 & $2.20 \times 10^{-4}$ & $15.23 \pm 0.76$ & $9.69 \pm 0.24$ & $10.45 \pm 0.56$ & $1.37 \pm 0.43$ \\
\hline $\mathbf{H}_{2}^{33} \mathbf{S}$ & $3_{2,1}-3_{1,2}$ & 369170.4 & 154.4 & $2.55 \times 10^{-5}$ & $7.31 \pm 1.32$ & $11.26 \pm 1.40$ & $16.12 \pm 3.71$ & $0.43 \pm 0.13$ \\
\hline $\mathbf{H}_{2}^{24} \mathbf{S}$ & $3_{2,1}-3_{1,2}$ & 369246.1 & 154.3 & $1.91 \times 10^{-4}$ & $8.92 \pm 0.63$ & $11.15 \pm 0.42$ & $11.54 \pm 1.13$ & $0.73 \pm 0.15$ \\
\hline${ }^{13} \mathbf{C S}^{\star}$ & $8-7$ & 369908.6 & 79.9 & $1.06 \times 10^{-3}$ & $11.87 \pm 0.45$ & $10.00 \pm 0.26$ & $14.02 \pm 0.59$ & $0.80 \pm 0.10$ \\
\hline $\mathrm{SO}_{2}^{\star}$ & $9_{6,4}-10_{5,5}$ & 370108.6 & 129.7 & $4.19 \times 10^{-5}$ & $28.15 \pm 0.43$ & $11.41 \pm 0.09$ & $12.53 \pm 0.23$ & $2.11 \pm 0.12$ \\
\hline U-line & & 370499.6 & & & $4.27 \pm 0.58$ & $10.94 \pm 1.41$ & $22.64 \pm 4.0$ & $0.18 \pm 0.06$ \\
\hline${ }^{34} \mathrm{SO}$ & $4_{4}-3_{4}$ & 370931.7 & 33.4 & $1.45 \times 10^{-5}$ & $2.55 \pm 0.39$ & $9.63 \pm 1.24$ & $13.90 \pm 2.46$ & $0.17 \pm 0.08$ \\
\hline Atmospheric & & 371036.0 & & & & & & \\
\hline $\mathrm{SO}_{2}{ }^{\star}$ & $6_{3,3}-5_{2,4}$ & 371172.5 & 41.4 & $3.55 \times 10^{-4}$ & $81.33 \pm 0.57$ & $9.89 \pm 0.05$ & $15.66 \pm 0.13$ & $4.88 \pm 0.13$ \\
\hline
\end{tabular}


Table A.1. continued.

\begin{tabular}{lllllllll}
\hline \hline Molecule & Transition & $\begin{array}{l}\text { Freq. } \\
(\mathrm{MHz})\end{array}$ & $\begin{array}{l}E_{\text {up }} \\
(\mathrm{K})\end{array}$ & \multicolumn{1}{l}{$\begin{array}{l}A_{\mathrm{ij}} \\
\left(\mathrm{s}^{-1}\right)\end{array}$} & $\begin{array}{l}\int T_{\mathrm{mb}} \mathrm{d} v \\
\left(\mathrm{~K} \mathrm{~km} \mathrm{~s}^{-1}\right)\end{array}$ & $\begin{array}{l}V_{\mathrm{LSR}} \\
\left(\mathrm{km} \mathrm{s}^{-1}\right)\end{array}$ & $\begin{array}{l}F W H M \\
\left(\mathrm{~km} \mathrm{~s}^{-1}\right)\end{array}$ & $\begin{array}{l}T_{\text {peak }} \\
(\mathrm{K})\end{array}$ \\
\hline $\mathbf{S O}_{2}, v_{2}=1$ & $21_{4,18}-21_{3,19}$ & 371264.8 & 1017.1 & $4.24 \times 10^{-4}$ & $4.11 \pm 0.71$ & $11.59 \pm 1.25$ & $13.72 \pm 3.49$ & $0.28 \pm 0.08$ \\
$\mathbf{C H}_{3} \mathbf{O H}^{\star}$ & $17_{1}-17_{0} \mathrm{~A}^{\mp}$ & 371415.7 & 372.4 & $2.52 \times 10^{-4}$ & $5.04 \pm 0.41$ & $9.57 \pm 0.62$ & $14.45 \pm 1.14$ & $0.33 \pm 0.09$ \\
${ }^{33} \mathbf{S O}_{2}$ & $25_{4,22}-25_{3,23}$ & 371804.6 & 343.0 & $1.35 \times 10^{-6}$ & $1.89 \pm 0.34$ & $10.69 \pm 0.85$ & $8.79 \pm 1.51$ & $0.20 \pm 0.08$ \\
$\mathbf{o - H}_{2} \mathbf{C S}^{\star}$ & $11_{1,11}-10_{1,10}$ & 371847.4 & 120.3 & $7.72 \times 10^{-4}$ & $10.58 \pm 0.28$ & $9.06 \pm 0.18$ & $13.18 \pm 0.36$ & $0.75 \pm 0.09$ \\
$\mathbf{S}^{17} \mathbf{O}$ & $9_{9}-8_{8}$ & 372113.6 & 102.5 & $6.33 \times 10^{-4}$ & $3.06 \pm 0.48$ & $9.84 \pm 0.95$ & $11.42 \pm 1.78$ & $0.25 \pm 0.09$ \\
$\mathbf{H N C O}^{34} \mathbf{S O}_{2}$ & $17_{1,17}-16_{1,16}$ & 372221.0 & 204.1 & $7.46 \times 10^{-4}$ & $8.13 \pm 0.64$ & $11.96 \pm 0.35$ & $9.23 \pm 0.83$ & $0.83 \pm 0.08$ \\
$\mathbf{N}_{2} \mathbf{H}^{+\star}$ & $13_{7,7}-14_{6,8}$ & 372279.8 & 199.7 & $6.00 \times 10^{-5}$ & $1.73 \pm 0.37$ & $11.74 \pm 0.50$ & $6.30 \pm 2.23$ & $0.26 \pm 0.05$ \\
$\mathbf{U}-l i n e^{c}$ & $4-3$ & 372672.5 & 44.7 & $3.33 \times 10^{-3}$ & $54.14 \pm 0.33$ & $7.44 \pm 0.04$ & $12.82 \pm 0.09$ & $3.97 \pm 0.20$ \\
$\mathbf{S O}^{18} \mathbf{O}$ & $22_{0,22}-21_{1,21}$ & 372869.7 & & & $7.92 \pm 0.41$ & $9.33 \pm 0.26$ & $10.05 \pm 0.64$ & $0.74 \pm 0.11$ \\
$\mathbf{S O}^{\star}$ & $4_{4}-3_{4}$ & 373344.1 & 221.2 & $6.69 \times 10^{-4}$ & $4.18 \pm 0.70$ & $13.21 \pm 0.90$ & $10.93 \pm 2.26$ & $0.36 \pm 0.09$ \\
$\mathbf{H N C O}^{1}$ & $17_{0,17}-16_{0,16}$ & 373600.7 & 161.4 & $7.54 \times 10^{-4}$ & $21.05 \pm 0.55$ & $10.73 \pm 0.15$ & $11.84 \pm 0.35$ & $1.67 \pm 0.13$ \\
\hline
\end{tabular}
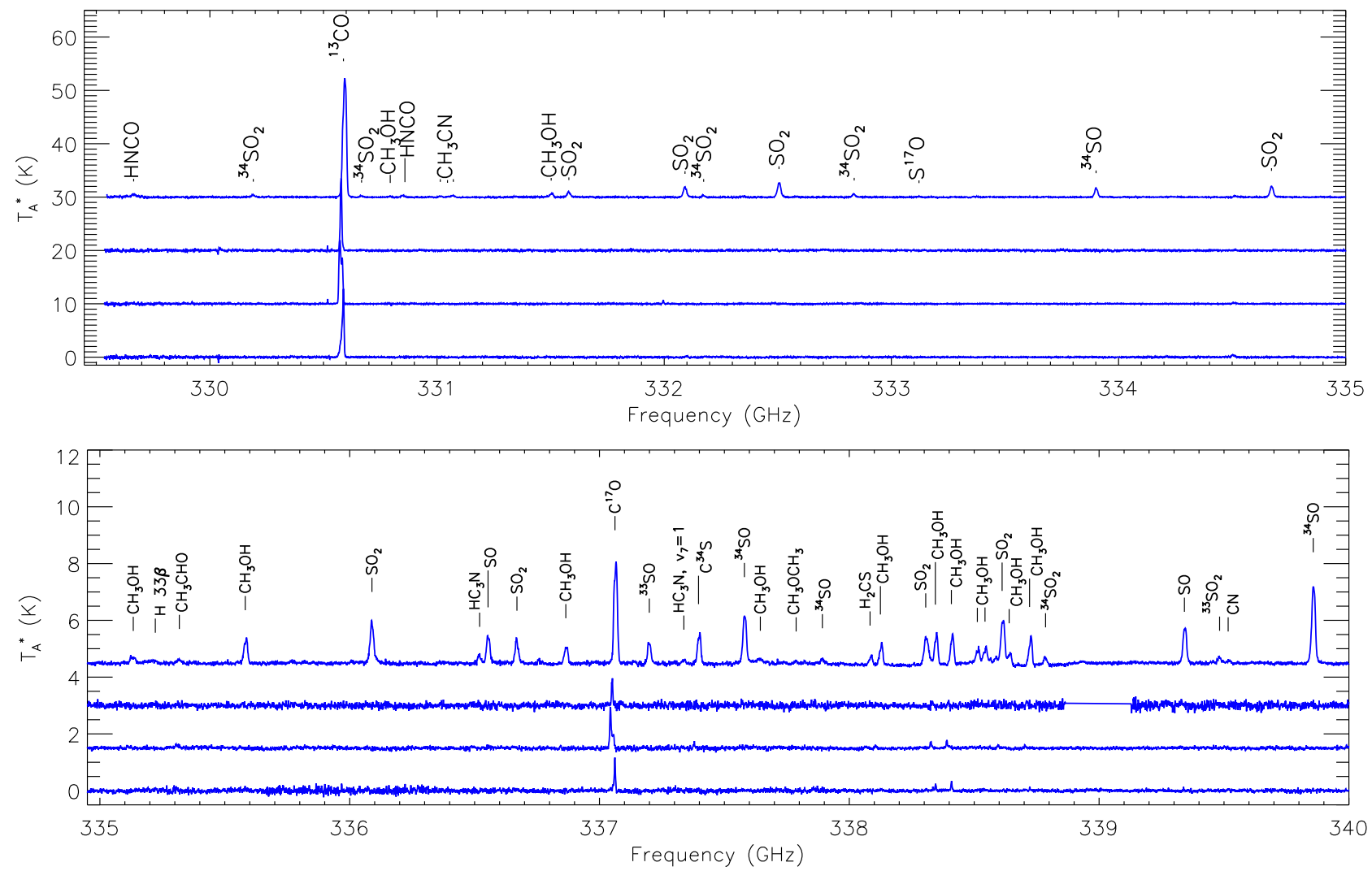

Fig. A.1. Identified lines toward the (from bottom to top) eastern tail, northern clump, southwest clump regions, and the center of W49A in the frequency range between 330 and $335 \mathrm{GHz}$ and 335 and $340 \mathrm{GHz}$. 

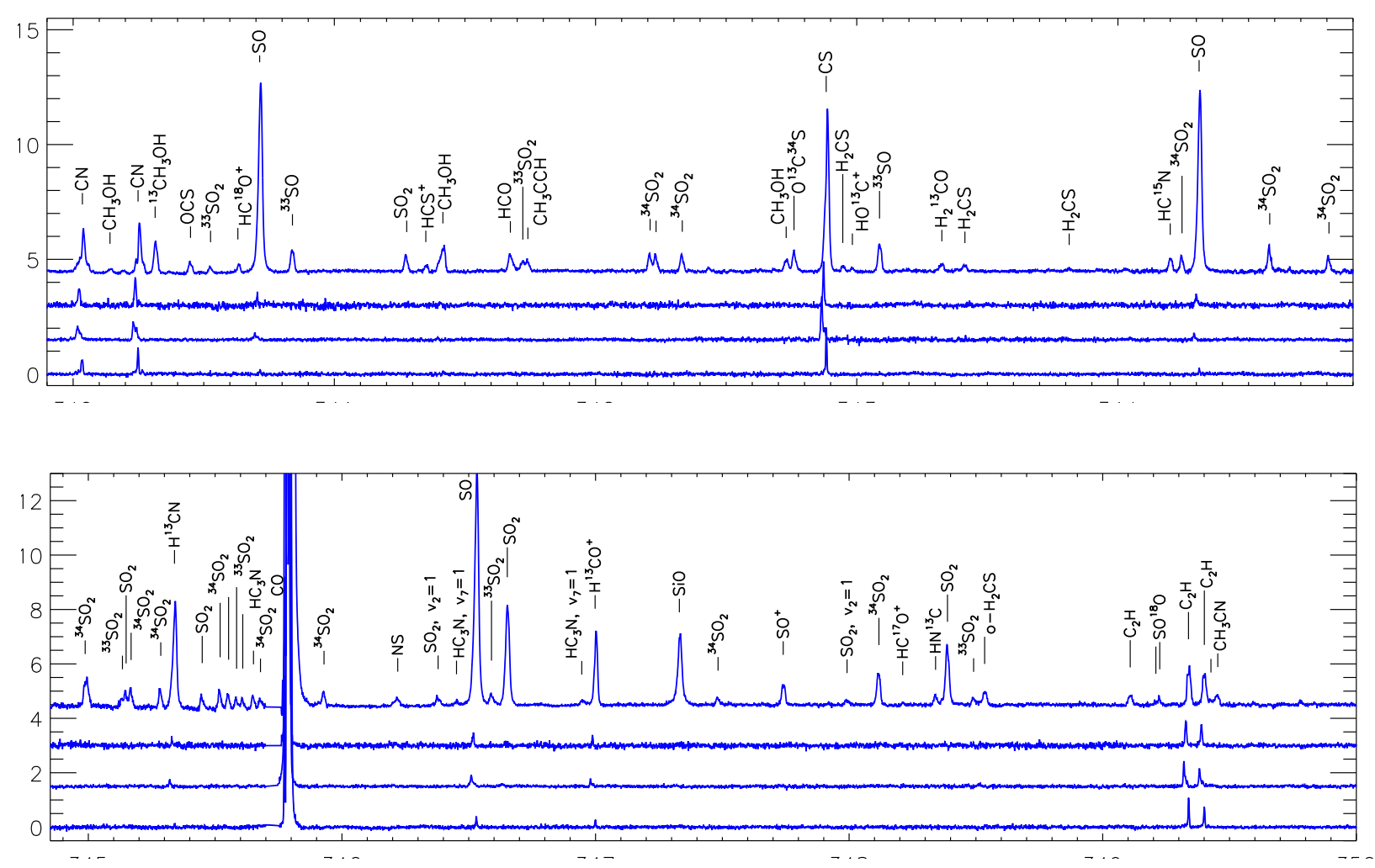

Fig. A.2. Identified lines toward the (from bottom to top) eastern tail, northern clump, southwest clump regions, and the center of W49A in the frequency range between 340 and $345 \mathrm{GHz}$ and 345 and $350 \mathrm{GHz}$.


Fig. A.3. Identified lines toward the (from bottom to top) eastern tail, northern clump, southwest clump regions, and the center of W49A in the frequency range between 350 and $355 \mathrm{GHz}$ and 355 and $360 \mathrm{GHz}$. 

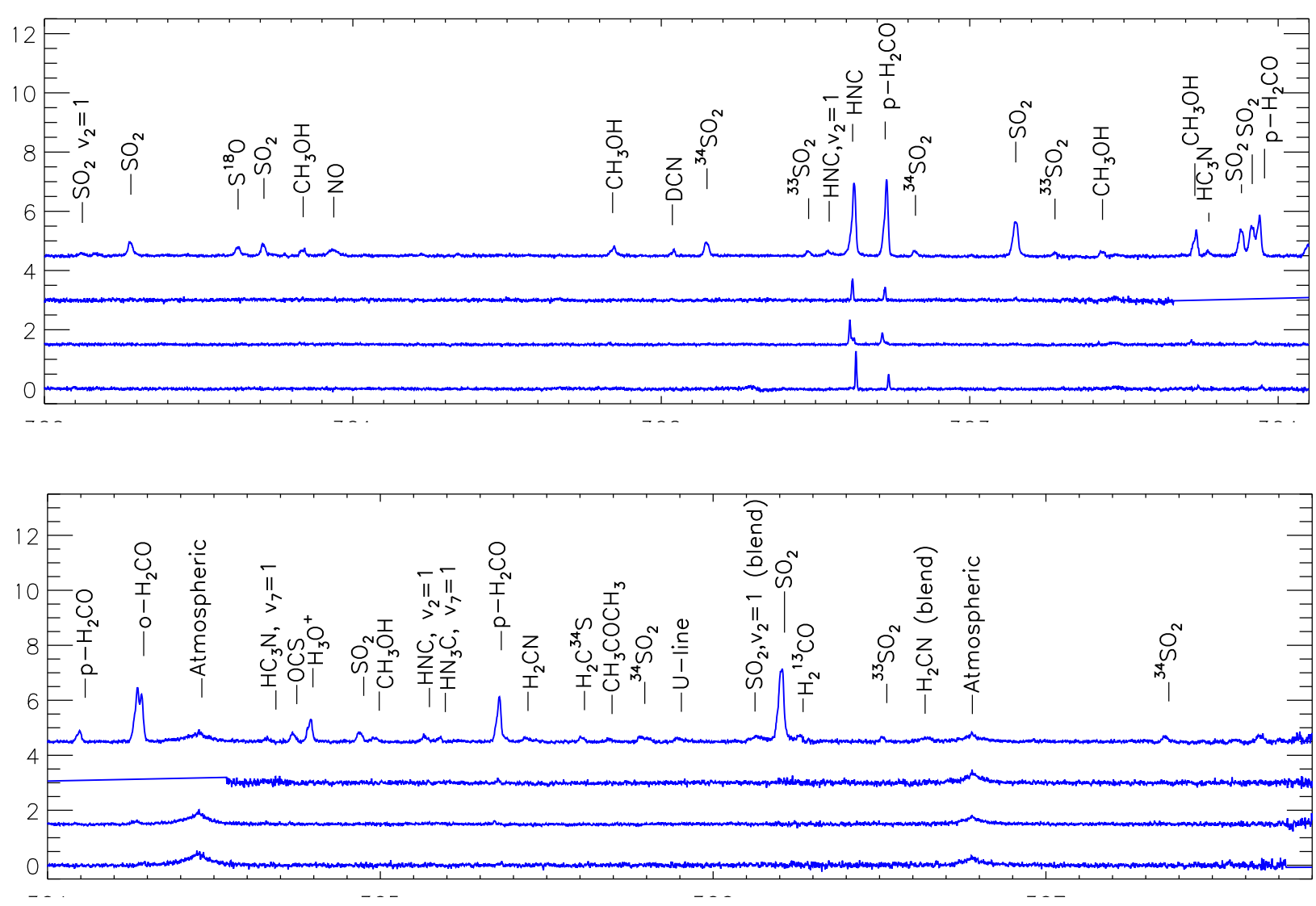

Fig. A.4. Identified lines toward the (from bottom to top) eastern tail, northern clump, southwest clump regions, and the center of W49A in the frequency range between 360 and $364 \mathrm{GHz}$ and 364 and $368 \mathrm{GHz}$.

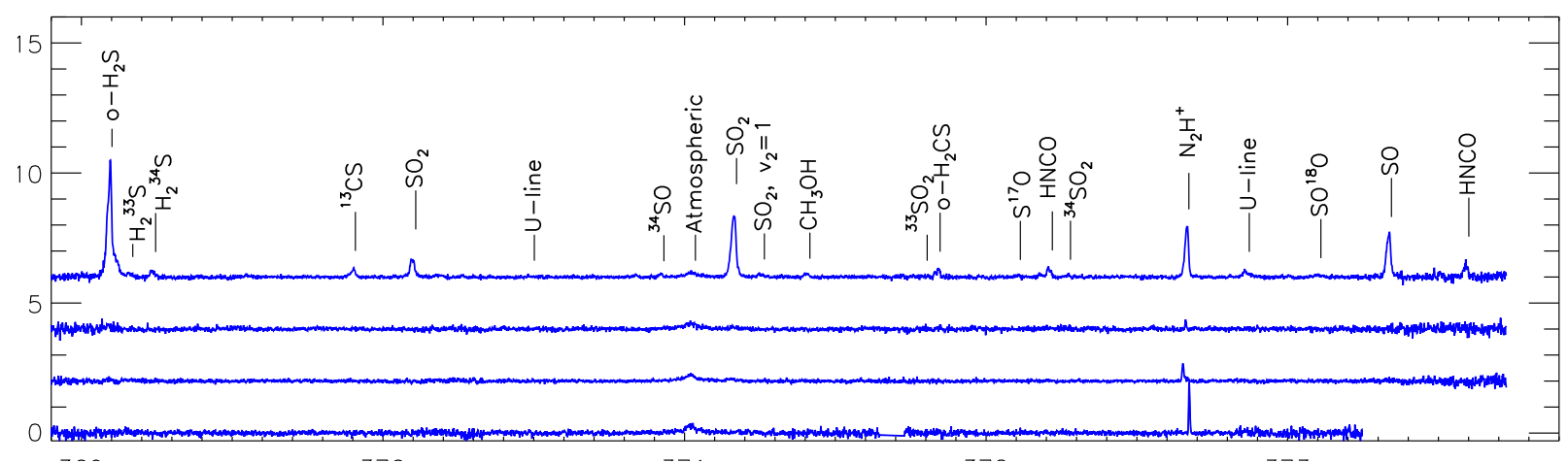

Fig. A.5. Identified lines toward the (from bottom to top) eastern tail, northern clump, southwest clump regions, and the center of of W49A in the frequency range between $369 \mathrm{GHz}$ and $374 \mathrm{GHz}$. 